\title{
Total Synthesis of Casuarinin
}

Shinnosuke Wakamori, ${ }^{*}$ Shintaro Matsumoto, Reina Kusuki, Kazutada Ikeuchi, ${ }^{\ddagger}$ Hidetoshi Yamada*,†

School of Science and Technology, Kwansei Gakuin University, 2-1 Gakuen, Sanda, Hyogo 669-1337, Japan

*Corresponding Author: (SW) shinnosuke1010@hotmail.co.jp

${ }^{\dagger}$ Deceased on November 23, 2019. This paper is dedicated to the memory of Prof. Dr. Hidetoshi Yamada.

${ }^{\ddagger}$ Present address: Department of Chemistry, Faculty of Science, Hokkaido University, Sapporo 060-0810, Japan

\section{Table of Contents}

\section{Supplementary Table}

SI-01 Nonidentical ${ }^{1} \mathrm{H}$ and ${ }^{13} \mathrm{C}$ NMR spectra of the synthesized 1 to the reported data ${ }^{37} \quad$ S3

SI-02 Identical ${ }^{1} \mathrm{H}$ NMR data of the synthesized $\mathbf{3 0}$ with those reported ${ }^{37} \quad$ S5

\section{Experimental Section}

SI-03 General information $\quad$ S6

SI-04 Compound 21

$\begin{array}{lr}\text { SI-05 Compound } 22 & \text { S7 }\end{array}$

SI-06 Compound $23 \quad$ S8

SI-07 Compound $24+$ S8

SI-08 Compound 24 (demonstration of reproducibility) S9

SI-09 Compound 24 (without the use of dichloromethane as a solvent) S9

$\begin{array}{ll}\text { SI-10 Compound } 31 & \text { S10 }\end{array}$

$\begin{array}{ll}\text { SI-11 Compound } 25 & \text { S11 }\end{array}$

SI-12 Compound $26 \quad$ S12

$\begin{array}{ll}\text { SI-13 Compound } 27 & \text { S13 }\end{array}$

SI-14 Compound 27 (unsuccess conditions) $\quad$ S13

$\begin{array}{lr}\text { SI-15 Compound } 28 & \text { S14 }\end{array}$

\begin{tabular}{l|l} 
SI-16 Compound 29 & S15
\end{tabular}

SI-17 Compound 29 (neutralized at workup) $\quad$ S15

$\begin{array}{lr}\text { SI-18 Casuarinin (1) } & \text { S16 }\end{array}$ 


\section{Spectra}

SI-20 Compound 21

S17

SI-21 Compound 22

S18

SI-22 Compound 23

S19

SI-23 Compound 23 (recovered)

S20

SI-24 Compound 24

S21

SI-25 Compound 31

S22

SI-26 Compound 25

S23

SI-27 Compound 26

S24

SI-28 Compound 27

S25

SI-29 Compound 28

S25

SI-30 Compound 29

S26

SI-31 Casuarinin (1)

S27

SI-32 Compound 30

S28

SI-33 Ratio in $\mathbf{2 3}$ and $\mathbf{2 4}$ in the incompletely separated fraction (see SI-09)

S29

SI-34 Comparison spectra of $\mathbf{2 3}$ with that of $\mathbf{2 3}$ (recovered)

S30

SI-35 Ratio in the sulfoxides (see SI-13)

S33

SI-36 Ratio in the oximes (see SI-15)

S33

SI-37 MS spectra of the hydrolysate of $\mathbf{2 9}$

S34

Selected Reference

S35 


\section{Supplementary Table}

SI-01 Nonidentical ${ }^{1} \mathrm{H}$ and ${ }^{13} \mathrm{C}$ NMR spectra of the synthesized 1 to the reported data ${ }^{37}$

\begin{tabular}{|c|c|c|c|}
\hline $\begin{array}{l}{ }^{1} \text { H NMR } \\
\text { Position }\end{array}$ & $\begin{array}{l}\delta \text { (Synthesized) } \\
500 \mathrm{MHz}, \text { acetone- } d_{6}\end{array}$ & $\begin{array}{l}\delta \text { (Reported, Ref 37) } \\
200 \mathrm{MHz} \text {, acetone- } d_{6}\end{array}$ & $\begin{array}{c}\Delta \delta \text { (Synthesized) - } \\
\delta \text { (Reported) }\end{array}$ \\
\hline \multirow{7}{*}{ sugar } & $4.07(\mathrm{~d}, J=13.0 \mathrm{~Hz}, \mathrm{H}-6)$ & $4.06(\mathrm{~d}, J=13 \mathrm{~Hz}, \mathrm{H}-6)$ & +0.01 \\
\hline & $4.67(\mathrm{dd}, J=5.0,2.0 \mathrm{~Hz}, \mathrm{H}-2)$ & $4.67(\mathrm{dd}, J=5,2 \mathrm{~Hz}, \mathrm{H}-2)$ & \pm 0.00 \\
\hline & $4.82(\mathrm{dd}, J=13.0,3.0 \mathrm{~Hz}, \mathrm{H}-6)$ & $4.18(\mathrm{dd}, J=13,3 \mathrm{~Hz}, \mathrm{H}-6)$ & +0.64 \\
\hline & $5.31(\mathrm{dd}, J=8.5,3.0 \mathrm{~Hz}, \mathrm{H}-5)$ & \multirow{3}{*}{5.39 (br s, H-3-5) } & -0.08 \\
\hline & $5.38(\mathrm{dd}, J=2.0,2.0 \mathrm{~Hz}, \mathrm{H}-3)$ & & -0.01 \\
\hline & $5.40(\mathrm{dd}, J=8.5,2.0 \mathrm{~Hz}, \mathrm{H}-4)$ & & +0.01 \\
\hline & $5.61(\mathrm{~d}, J=5.0 \mathrm{~Hz}, \mathrm{H}-1)$ & $5.64(\mathrm{~d}, J=5 \mathrm{~Hz}, \mathrm{H}-1)$ & -0.03 \\
\hline \multirow{3}{*}{ HHDP* } & $6.47(\mathrm{~s})$ & $6.50(\mathrm{~s})$ & -0.03 \\
\hline & $6.53(\mathrm{~s})$ & $6.56(\mathrm{~s})$ & -0.03 \\
\hline & $6.77(\mathrm{~s})$ & $6.78(\mathrm{~s})$ & -0.01 \\
\hline $\mathrm{G}^{* *}$ & $7.09(\mathrm{~s}, 2 \mathrm{H})$ & $7.12(\mathrm{~s})$ & -0.03 \\
\hline
\end{tabular}

*HHDP $=$ hexahydroxydiphenoyl group, ${ }^{*} \mathrm{G}=$ galloyl group 


\begin{tabular}{|c|c|c|c|}
\hline $\begin{array}{l}{ }^{13} \text { C NMR } \\
\text { Position }\end{array}$ & $\begin{array}{l}\delta \text { (Synthesized) } \\
126 \mathrm{MHz} \text {, acetone- } d_{6}\end{array}$ & $\begin{array}{l}\delta \text { (Reported, Ref } 37) \\
50 \mathrm{MHz} \text {, acetone- } d_{6}\end{array}$ & $\begin{array}{c}\Delta \delta \text { (Synthesized) - } \\
\delta \text { (Reported) }\end{array}$ \\
\hline \multirow{6}{*}{ sugar } & $64.5(t, C-6)$ & 64.5 & \pm 0.0 \\
\hline & $67.7(\mathrm{~d}, \mathrm{C}-1)$ & 67.8 & -0.1 \\
\hline & $69.9(\mathrm{~d}, \mathrm{C}-3)$ & 69.8 & +0.1 \\
\hline & $71.2(\mathrm{~d}, \mathrm{C}-5)$ & 71.1 & +0.1 \\
\hline & $74.2(\mathrm{~d}, \mathrm{C}-4)$ & 74.3 & -0.1 \\
\hline & 76.7 (d, C-2) & 76.7 & \pm 0.0 \\
\hline \multirow{3}{*}{ HHDP } & 105.2 (d) & 105.7 & -0.5 \\
\hline & 107.1 (d) & 107.5 & -0.4 \\
\hline & $108.3(d)$ & 108.7 & -0.4 \\
\hline $\mathrm{G}$ & $110.2(\mathrm{~d}, 2 \mathrm{C})$ & $110.5(2 \mathrm{C})$ & -0.3 \\
\hline \multirow{6}{*}{ HHDP } & $115.3(\mathrm{~s})$ & 115.4 & -0.1 \\
\hline & $115.9(\mathrm{~s})$ & 116.1 & -0.2 \\
\hline & $116.0(\mathrm{~s})$ & 116.3 & -0.3 \\
\hline & $116.4(\mathrm{~s})$ & 116.5 & -0.1 \\
\hline & $117.0(\mathrm{~s})$ & 117.4 & -0.4 \\
\hline & $120.7(\mathrm{~s})$ & 121.0 & -0.3 \\
\hline $\mathrm{G}$ and & $121.1(\mathrm{~s})$ & 121.4 & -0.3 \\
\hline HHDP & $125.1(\mathrm{~s})$ & 125.4 & -0.3 \\
\hline \multirow{6}{*}{ HHDP } & $127.2(\mathrm{~s})$ & 127.5 & -0.3 \\
\hline & $127.7(\mathrm{~s})$ & 128.0 & -0.3 \\
\hline & $134.8(\mathrm{~s})$ & 135.3 & -0.5 \\
\hline & $135.9(\mathrm{~s})$ & 136.3 & -0.4 \\
\hline & $136.8(\mathrm{~s})$ & 137.3 & -0.5 \\
\hline & $138.4(\mathrm{~s})$ & 138.8 & -0.4 \\
\hline G & $139.2(\mathrm{~s})$ & 139.6 & -0.4 \\
\hline \multirow{7}{*}{ HHDP } & $143.8(\mathrm{~s})$ & 144.1 & -0.3 \\
\hline & $144.0(\mathrm{~s})$ & 144.4 & -0.4 \\
\hline & $144.6(\mathrm{~s})$ & 145.0 & -0.4 \\
\hline & $144.8(\mathrm{~s})$ & 145.2 & -0.4 \\
\hline & $145.1(\mathrm{~s})$ & 145.7 & -0.6 \\
\hline & $145.2(\mathrm{~s})$ & 145.7 & -0.5 \\
\hline & $145.7(\mathrm{~s})$ & 146.4 & -0.7 \\
\hline G & $146.0(\mathrm{~s}, 2 \mathrm{C})$ & $146.4(2 \mathrm{C})$ & -0.4 \\
\hline \multirow{2}{*}{ HHDP } & $146.4(\mathrm{~s})$ & 146.8 & -0.4 \\
\hline & $164.2(\mathrm{~s})$ & 165.0 & -0.8 \\
\hline G & $165.7(\mathrm{~s})$ & 166.4 & -0.7 \\
\hline \multirow{3}{*}{ HHDP } & $168.6(\mathrm{~s})$ & 169.2 & -0.6 \\
\hline & $168.9(\mathrm{~s})$ & 169.5 & -0.6 \\
\hline & $169.7(\mathrm{~s})$ & 170.3 & -0.6 \\
\hline
\end{tabular}


SI-02 Identical ${ }^{1} \mathrm{H}$ NMR data of the synthesized 30 with those reported ${ }^{37}$

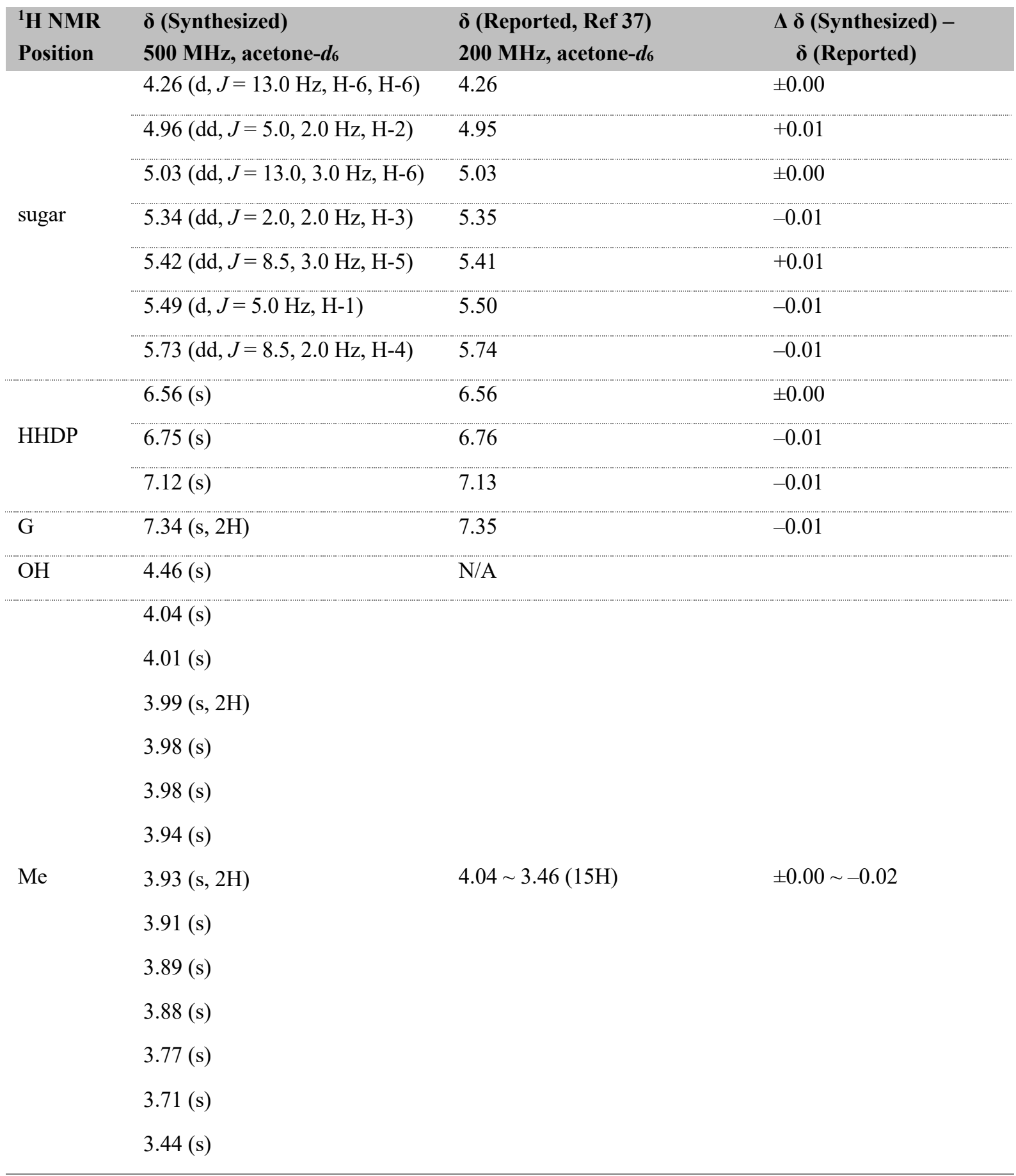




\section{Experimental Section}

\section{SI-03 General information}

All commercially available reagents were used as received. All moisture and air sensitive reactions were carried out in glassware equipped with rubber septa (or a septum) under the positive pressure of nitrogen. When necessary, the glassware was dried under reduced pressure by heating with a heat-gun and solvents were distilled prior to use. The reaction mixture was magnetically stirred. For reactions that require heating, an oil bath or a personal organic synthesizer (EYELA PPM-5512) were utilized. Concentration was performed under reduced pressure.

The reactions were monitored by thin layer chromatography (TLC) and mass spectra (MS). Anhydrous $\mathrm{MgSO}_{4}$ was used to dry organic layers after extraction, and it was removed by filtration through a cotton pad. The filtrate was concentrated and subjected to further purification protocols if necessary. This sequence was represented as "the general drying procedure" in the following experimental methods. TLC was performed on Merck precoated silica gel 60 F-254 plates. Spots were visualized by exposure to UV light, or by immersion into a solution of $2 \%$ anisaldehyde and $5 \% \mathrm{H}_{2} \mathrm{SO}_{4}$ in ethanol followed by heating at ca. $200{ }^{\circ} \mathrm{C}$.

Column chromatography (CC) was performed on Merck silica gel 60 (63-200 or 40-63 $\mu \mathrm{m})$ and Kanto Chemical silica gel $60 \mathrm{~N}$ (Spherical, neutral, 40-50 or 63-210 $\mu \mathrm{m}$ ), for ordinary phase or Nacalai Tesque Cosmosil 140C18-PREP for reverse phase. The other carrier materials were noted in each case. The melting points were determined using a Yanagimoto micro-melting point apparatus and uncorrected. Optical rotations were determined using a JASCO DIP-370 polarimeter with a $100 \mathrm{~mm}$ cell with transmitting the sodium D-line. IR spectra were recorded on Shimazu IRAffinity-1S with an attenuated total reflectance (ATR) sampling unit, and the major absorbance bands are all reported in wavenumbers $\left(\mathrm{cm}^{-1}\right)$. High-resolution (HR) mass spectra were obtained on a JEOL JMS-T100LC spectrometer for electrospray ionization (ESI). The data are reported in $\mathrm{m} / z$.

Nuclear magnetic resonance (NMR) spectra were recorded on JNM-ECX-500 (500 MHz for ${ }^{1} \mathrm{H}$ and 126 $\mathrm{MHz}$ for ${ }^{13} \mathrm{C}$ ) with either TMS or residual proton of deuterated solvent as internal reference in the indicated solvent in each parenthesis. The ${ }^{1} \mathrm{H}$ NMR spectroscopic data are indicated by a chemical shift $(\delta)$, with the multiplicity, the coupling constants, the integration, and the assignments in parentheses in this order. The multiplicities are abbreviated as s: singlet, $\mathrm{d}$ : doublet, t: triplet, q: quartet, m: multiplet, and br: broad. The ${ }^{13} \mathrm{C}$ NMR spectroscopic data are reported as the chemical shift $(\delta)$, with the hydrogen multiplicity obtained from the DEPT spectra and the assignments in parentheses. The multiplicities are abbreviated as $\mathrm{s}: \mathrm{C}, \mathrm{d}: \mathrm{CH}, \mathrm{t}: \mathrm{CH}_{2}$, and q: $\mathrm{CH}_{3}$. When the number of the carbon was more than one, the number was added in the parentheses. In the case that there are very close but individual signals without overlap and when rounding chemical shifts of the signals at the second decimal place provides same values, their chemical shifts were written up to two decimal places.

\section{SI-04 Compound 21}

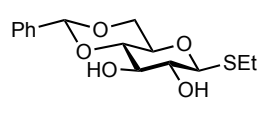

20

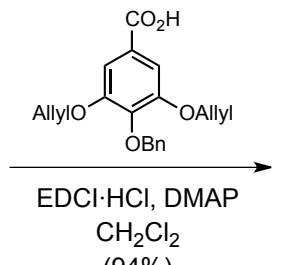

(94\%)

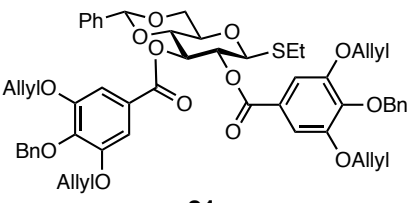

21

A mixture of diol $\mathbf{2 0}^{24}(5.00 \mathrm{~g}, 16.0 \mathrm{mmol}), \mathrm{Bn} /$ Allyl protected gallic acid ${ }^{25}$ (15.0 g, $\left.40.0 \mathrm{mmol}\right)$, DMAP $(2.93 \mathrm{~g}, 24.0 \mathrm{mmol})$, and EDCI hydrochloride $(15.3 \mathrm{~g}, 80.0 \mathrm{mmol})$ in dichloromethane $(800 \mathrm{~mL})$ was stirred for $13 \mathrm{~h}$ at rt. After removal of dichloromethane by concentration of the reaction mixture, to the resulting residue were added diethyl ether and brine. The mixture was extracted with diethyl ether. The organic layer was washed with brine. After the general drying procedure, the crude product was purified by CC (silica gel $150 \mathrm{~g}$, hexane/ethyl acetate $=5 / 1$ to 3/1) and subsequent recrystallization (hexane/ethyl acetate) to afford 21 (13.9 g, 15.0 mmol, $94 \%$ yield) as a colorless crystal: $\mathrm{mp} 114-117^{\circ} \mathrm{C}$; $[\alpha]_{\mathrm{D}}{ }^{23}+66\left(c 0.20, \mathrm{CHCl}_{3}\right)$; IR (ATR) v 3090, 3065, 
3030, 2928, 2866, 1721, 1585, 1499, 1454, 1422, 1331, 1200, 1098, 1013, 991, 926, 754, 735, $698 \mathrm{~cm}^{-1}$; ${ }^{1} \mathrm{H}$ NMR $\left(500 \mathrm{MHz}, \mathrm{CDCl}_{3}, 23.3{ }^{\circ} \mathrm{C}\right) \delta$ 7.47-7.42 (m, 6H, Ar), 7.34-7.27 (m, 9H, Ar), $7.18(\mathrm{~s}, 2 \mathrm{H}, \mathrm{G}), 7.16(\mathrm{~s}, 2 \mathrm{H}$, G), 6.03 (ddt, $J=17.0,10.0,3.0 \mathrm{~Hz}, 2 \mathrm{H}$, Allyl), 6.01 (ddt, $J=17.0,10.0,3.0 \mathrm{~Hz}, 2 \mathrm{H}$, Allyl), 5.74 (dd, $J=10.0$, $10.0 \mathrm{~Hz}, 1 \mathrm{H}, \mathrm{H}-3), 5.55$ (s, 1H, PhCH), 5.44 (dd, $J=10.0,10.0 \mathrm{~Hz}, 1 \mathrm{H}, \mathrm{H}-2), 5.40$ (ddt, J=17.0, 1.0, 1.0 Hz, 2H, Allyl), 5.39 (ddt, $J=17.0,1.0,1.0 \mathrm{~Hz}, 2 \mathrm{H}$, Allyl), 5.26 (ddt, $J=10.0,1.0,1.0 \mathrm{~Hz}, 2 \mathrm{H}$, Allyl), 5.24 (ddt, $J=10.0$, 1.0, $1.0 \mathrm{~Hz}, 2 \mathrm{H}, \mathrm{Allyl}), 5.08$ (d, $J=11.5 \mathrm{~Hz}, 1 \mathrm{H}, \mathrm{Bn}), 5.06$ (d, $J=11.5 \mathrm{~Hz}, 1 \mathrm{H}, \mathrm{Bn}), 5.06$ (d, $J=11.5 \mathrm{~Hz}, 1 \mathrm{H}$, $\mathrm{Bn}), 5.04(\mathrm{~d}, J=11.5 \mathrm{~Hz}, 1 \mathrm{H}, \mathrm{Bn}), 4.81(\mathrm{~d}, J=10.0 \mathrm{~Hz}, 1 \mathrm{H}, \mathrm{H}-1), 4.56-4.53(\mathrm{~m}, 8 \mathrm{H}, \mathrm{Bn}$ and Allyl), 4.44 (dd, $J=$ 10.0, $5.0 \mathrm{~Hz}, 1 \mathrm{H}, \mathrm{H}-6), 3.91$ (dd, $J=10.0,10.0 \mathrm{~Hz}, 1 \mathrm{H}, \mathrm{H}-4), 3.87$ (dd, $J=10.0,10.0 \mathrm{~Hz}, 1 \mathrm{H}, \mathrm{H}-6), 3.74$ (ddd, $J$ $=10.0,10.0,5.0 \mathrm{~Hz}, 1 \mathrm{H}, \mathrm{H}-5), 2.79(\mathrm{dq}, J=12.5,7.5 \mathrm{~Hz}, 1 \mathrm{H}, \mathrm{SEt}), 2.76(\mathrm{dq}, J=12.5,7.5 \mathrm{~Hz}, 1 \mathrm{H}, \mathrm{SEt}), 1.29$ (dd, $J=7.5,7.5 \mathrm{~Hz}, 3 \mathrm{H}, \mathrm{SEt}) ;{ }^{13} \mathrm{C} \mathrm{NMR}\left(126 \mathrm{MHz}, \mathrm{CDCl}_{3}, 23.9^{\circ} \mathrm{C}\right) \delta 165.4(\mathrm{~s}, \mathrm{G}), 165.1$ (s, G), 152.3 (s, G), 152.2 (s, G), 142.4 (s, G), 142.3 (s, G), 137.5 (s, G), 136.7 (s, G), 132.9 (d, 2C, Allyl), 132.8 (d, 2C, Allyl), 129.1 (d, $\mathrm{Bn}$ ), 128.3-128.2 (overlapping 14 doublets and 3 singlets: 3 peaks were observed, $\mathrm{Bn}$ and $\mathrm{Ph}$ ), $126.2(\mathrm{~d}, 2 \mathrm{C}, \mathrm{Bn})$, 124.3 (s, G), 124.0 (s, G), 117.8 (t, 4C, Allyl), 108.9 (d, 2C, G), 101.5 (d, PhCH), 84.5 (d, C-1), 78.7 (d, C-4), 74.9 (t, 2C, Bn), 73.5 (d, C-3), 71.3 (d, C-2), 71.1 (d, C-5), 69.9 (t, 4C, Allyl), 68.6 (t, C-6), 24.5 (t, SEt), 14.9 (q, $\mathrm{SEt}$ ); HRMS (ESI) $\mathrm{m} / z[\mathrm{M}+\mathrm{Na}]^{+}$calcd for $\mathrm{C}_{55} \mathrm{H}_{56} \mathrm{O}_{13} \mathrm{SNa} 979.3339$, found 979.3310 .

\section{SI-05 Compound 22}

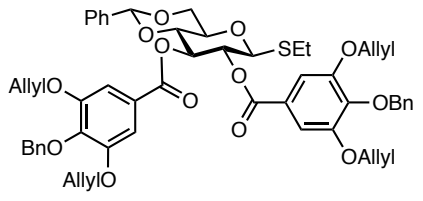

21

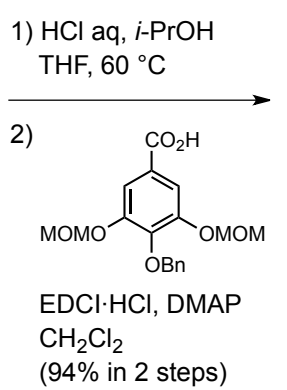

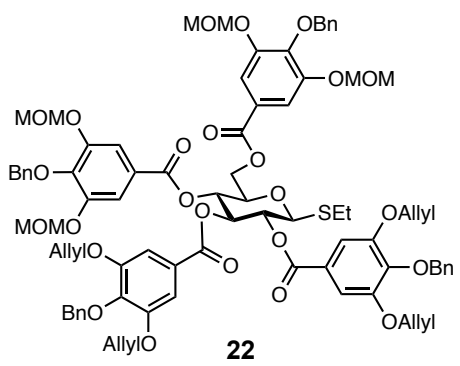

22

To a solution of $21(6.37 \mathrm{~g}, 6.86 \mathrm{mmol})$ in THF $(69.0 \mathrm{~mL})$ was added a mixture of 2-propanol and conc. hydrochloric acid (v/v 50/1, $69.0 \mathrm{~mL}$ ). The mixture was stirred for $20 \mathrm{~h}$ at $\mathrm{rt}$. After addition of sat. sodium hydrogen carbonate aq, the mixture was extracted with ethyl acetate. The organic layer was washed with brine. The general drying procedure provided a crude product, which was used for the subsequent transformation without further purification. A mixture of the crude product, Bn/MOM protected gallic acid ${ }^{26}$ (5.26 g, 15.1 mmol), DMAP $(1.26 \mathrm{~g}, 10.3 \mathrm{mmol})$, and EDCI hydrochloride $(6.56 \mathrm{~g}, 34.3 \mathrm{mmol})$ in dichloromethane $(343 \mathrm{~mL})$ was stirred for $13 \mathrm{~h}$ at rt. After removal of dichloromethane, to the residue was added diethyl ether and brine. The aqueous mixture was extracted with diethyl ether. The organic layer was washed with brine. After the general drying procedure, the crude product was purified by CC (silica gel $150 \mathrm{~g}$, hexane/ethyl acetate $=6 / 1$ to $2 / 1)$ to afford $22(9.90$ g, $6.47 \mathrm{mmol}, 94 \%$ yield in 2 steps) as a colorless amorphous solid: $[\alpha]_{\mathrm{D}}{ }^{24}+34\left(c 0.095, \mathrm{CHCl}_{3}\right)$; IR (ATR) $v$ $3088,3065,3030,2955,1726,1585,1497,1431,1327,1190,1155,1107,1045,924,754,735,698 \mathrm{~cm}^{-1} ;{ }^{1} \mathrm{H}$ NMR $\left(500 \mathrm{MHz}, \mathrm{CDCl}_{3}, 23.2^{\circ} \mathrm{C}\right) \delta 7.55(\mathrm{~s}, 2 \mathrm{H}, \mathrm{G}), 7.46(\mathrm{dd}, J=6.5,5.5 \mathrm{~Hz}, 4 \mathrm{H}, \mathrm{Bn}), 7.42(\mathrm{~s}, 2 \mathrm{H}, \mathrm{G}), 7.42(\mathrm{dd}$, $J=6.5,5.5 \mathrm{~Hz}, 4 \mathrm{H}, \mathrm{Bn}), 7.36-7.27$ (m, 12H, Bn), 7.20 (s, 2H, G), 7.05 (s, 2H, G), 6.03 (ddt, $J=17.0,11.0,5.0$ $\mathrm{Hz}, 2 \mathrm{H}$, Allyl), 5.95 (ddt, $J=17.0,11.0,5.0 \mathrm{~Hz}, 2 \mathrm{H}$, Allyl), 5.84 (dd, $J=10.0,10.0 \mathrm{~Hz}, 1 \mathrm{H}, \mathrm{H}-3$ ), 5.54 (dd, $J=$ $10.0,10.0 \mathrm{~Hz}, 1 \mathrm{H}, \mathrm{H}-2), 5.45$ (dd, $J=10.0,10.0 \mathrm{~Hz}, 1 \mathrm{H}, \mathrm{H}-4), 5.41$ (dd, $J=17.0,1.0 \mathrm{~Hz}, 2 \mathrm{H}$, Allyl), 5.37 (dd, $J$ $=17.0,1.0 \mathrm{~Hz}, 2 \mathrm{H}$, Allyl), 5.27 (dd, $J=11.0,1.0 \mathrm{~Hz}, 2 \mathrm{H}$, Allyl), 5.23 (dd, $J=11.0,1.0 \mathrm{~Hz}, 2 \mathrm{H}$, Allyl), 5.20 (s, $\left.4 \mathrm{H}, \mathrm{CH}_{2}\right), 5.17-5.13\left(\mathrm{~m}, 6 \mathrm{H}, \mathrm{CH}_{2}\right), 5.08\left(\mathrm{~d}, J=11.5 \mathrm{~Hz}, 2 \mathrm{H}, \mathrm{CH}_{2}\right), 5.06\left(\mathrm{~d}, J=11.5 \mathrm{~Hz}, 2 \mathrm{H}, \mathrm{CH}_{2}\right), 5.01(\mathrm{~s}, 2 \mathrm{H}$, $\mathrm{CH}_{2}$ ), 4.83 (d, $\left.J=10.0 \mathrm{~Hz}, 1 \mathrm{H}, \mathrm{H}-1\right), 4.68$ (dd, $J=12.5,2.0 \mathrm{~Hz}, 1 \mathrm{H}, \mathrm{H}-6$ ), 4.57 (dd, $J=13.0,5.0 \mathrm{~Hz}, 2 \mathrm{H}$, Allyl), 4.55 (dd, $J=13.0,5.0 \mathrm{~Hz}, 2 \mathrm{H}$, Allyl), 4.49 (d, $J=5.0 \mathrm{~Hz}, 4 \mathrm{H}$, Allyl), 4.35 (dd, $J=12.5,6.0 \mathrm{~Hz}, 1 \mathrm{H}, \mathrm{H}-6), 4.16$ (ddd, $J=10.0,6.0,2.0 \mathrm{~Hz}, 1 \mathrm{H}, \mathrm{H}-5), 3.49$ (s, 6H, MOM), 3.45 (s, 6H, MOM), 2.78 (dq, $J=13.0,7.5 \mathrm{~Hz}, 1 \mathrm{H}$, $\mathrm{SEt}), 2.73(\mathrm{dq}, J=13.0,7.5 \mathrm{~Hz}, 1 \mathrm{H}, \mathrm{SEt}), 1.25(\mathrm{dd}, J=7.5,7.5 \mathrm{~Hz}, 3 \mathrm{H}, \mathrm{SEt}) ;{ }^{13} \mathrm{C} \mathrm{NMR}\left(126 \mathrm{MHz}, \mathrm{CDCl}_{3}\right.$, $\left.23.4{ }^{\circ} \mathrm{C}\right) \delta 165.5(\mathrm{~s}, \mathrm{G}), 165.4(\mathrm{~s}, \mathrm{G}), 164.9(\mathrm{~s}, \mathrm{G}), 164.7$ (s, G), 152.3 (s, G), 152.2 (s, G), 150.9 (s, 2C, G), 143.7 (s, G), 143.3 (s, G), 142.4 (s, G), 142.3 (s, G), 137.5 (s, 2C, G), 137.3 (s, G), 137.2 (s, G), 132.9 (d, 2C, Allyl), 132.8 (d, 2C, Allyl), 127.8-128.3 (overlapping 20 doublets and 4 singlets: 7 peaks were observed, Bn), 125.1 (s, G), 124.0 (s, 2C, G), 123.8 (s, G), 117.8 (t, 4C, Allyl), 112.3 (d, 4C, G), 109.0 (d, 2C, G), 108.7 (d, 2C, G), 95.5 (t, 2C, Allyl), 95.4 (t, 2C, Allyl), 83.8 (d, C-1), 76.4 (d, C-5), 75.2 (t, 2C, Bn), 74.91 (t, Bn), 74.86 (t, Bn), 74.4 (d, 
C-3), 70.9 (d, C-2), 69.9 (t, 2C, Allyl), 69.8 (t, 2C, Allyl), 69.6 (d, C-4), 63.5 (t, C-6), 56.4 (q, 4C, MOM), 24.4 (t, $\mathrm{SEt}$ ), 14.8 (q, SEt); HRMS (ESI) $\mathrm{m} / z$ [M + Na] $]^{+}$calcd for $\mathrm{C}_{84} \mathrm{H}_{88} \mathrm{O}_{25} \mathrm{SNa} 1551.5233$, found 1551.5218 .

\section{SI-06 Compound 23}
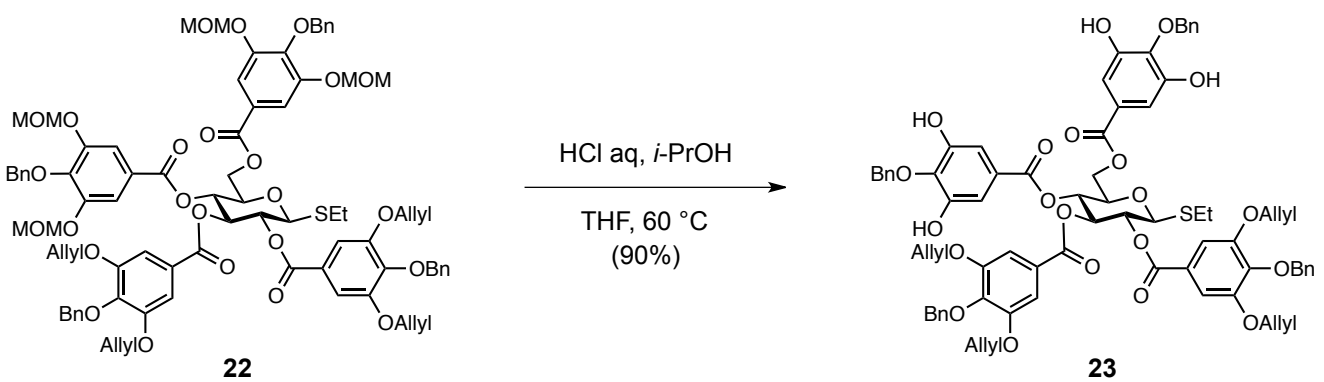

To a solution of $22(4.20 \mathrm{~g}, 2.75 \mathrm{mmol})$ in THF $(50.0 \mathrm{~mL})$ was added a mixture of 2-propanol and conc. hydrochloric acid $(\mathrm{v} / \mathrm{v} 50 / 1,50.0 \mathrm{~mL})$. The mixture was stirred for $16 \mathrm{~h}$ at $60{ }^{\circ} \mathrm{C}$. After addition of sat. sodium hydrogen carbonate aq at $\mathrm{rt}$, the mixture was extracted with ethyl acetate. The organic layer was washed with brine. After the general drying procedure, the crude product was purified by $\mathrm{CC}$ (silica gel $75 \mathrm{~g}$, hexane/ethyl acetate $=5 / 1$ to $3 / 2)$ to afford $23(3.34 \mathrm{~g}, 2.47 \mathrm{mmol}, 90 \%$ yield $)$ as a colorless amorphous solid: $[\alpha]_{\mathrm{D}}{ }^{24}+30(c 0.25$, $\mathrm{CHCl}_{3}$ ); IR (ATR) v 3422, 3086, 3030, 2959, 2872, 1722, 1589, 1456, 1433, 1333, 1198, 1109, 1057, 997, 754, $698 \mathrm{~cm}^{-1}$; ${ }^{1} \mathrm{H}$ NMR $\left(500 \mathrm{MHz}, \mathrm{CDCl}_{3}, 23.2{ }^{\circ} \mathrm{C}\right) \delta 7.44(\mathrm{~d}, J=7.0 \mathrm{~Hz}, 2 \mathrm{H}, \mathrm{Bn}), 7.39-7.22(\mathrm{~m}, 18 \mathrm{H}, \mathrm{Bn}), 7.21$ (s, 2H, G), 7.20 (s, 2H, G), 7.08 (s, 2H, G), 7.05 (s, 2H, G), 6.12 (br s, 1H, OH), 6.09 (br s, 1H, OH), 6.01 (ddt, $J=$ 17.0, 10.0, $5.0 \mathrm{~Hz}, 2 \mathrm{H}, \mathrm{Allyl}), 5.91$ (ddt, $J=17.0,10.0,5.0 \mathrm{~Hz}, 2 \mathrm{H}$, Allyl), 5.80 (dd, $J=10.0,10.0 \mathrm{~Hz}, 1 \mathrm{H}, \mathrm{H}-3$ ), $5.56(\mathrm{dd}, J=10.0,10.0 \mathrm{~Hz}, 1 \mathrm{H}, \mathrm{H}-4), 5.50(\mathrm{dd}, J=10.0,10.0 \mathrm{~Hz}, 1 \mathrm{H}, \mathrm{H}-2), 5.39$ (dd, $J=17.0,1.5 \mathrm{~Hz}, 2 \mathrm{H}$, Allyl), 5.30 (dd, $J=17.0,1.5 \mathrm{~Hz}, 2 \mathrm{H}$, Allyl), 5.24 (dd, $J=10.0,1.5 \mathrm{~Hz}, 2 \mathrm{H}$, Allyl), 5.16 (dd, $J=10.0,1.5 \mathrm{~Hz}, 2 \mathrm{H}$, Allyl), $5.14(\mathrm{~d}, J=12.0 \mathrm{~Hz}, 1 \mathrm{H}, \mathrm{Bn}), 5.11(\mathrm{~d}, J=12.0 \mathrm{~Hz}, 1 \mathrm{H}, \mathrm{Bn}), 5.08(\mathrm{~d}, J=12.0 \mathrm{~Hz}, 1 \mathrm{H}, \mathrm{Bn}), 5.05(\mathrm{~d}, J=12.0 \mathrm{~Hz}$, 1H, Bn), 5.05 (s, 2H, Bn), 4.98 (s, 2H, Bn), 4.83 (d, $J=10.0 \mathrm{~Hz}, 1 \mathrm{H}, \mathrm{H}-1), 4.59$ (dd, $J=13.0,4.5 \mathrm{~Hz}, 1 \mathrm{H}, \mathrm{H}-6$ ), 4.54 (d, $J=5.0 \mathrm{~Hz}, 4 \mathrm{H}$, Allyl), 4.49 (dd, $J=13.0,3.0 \mathrm{~Hz}, 1 \mathrm{H}, \mathrm{H}-6), 4.45$ (dd, $J=13.0,5.0 \mathrm{~Hz}, 2 \mathrm{H}$, Allyl), 4.42 (dd, $J=13.0,5.0 \mathrm{~Hz}, 2 \mathrm{H}$, Allyl), 4.07 (ddd, $J=10.0,4.5,3.0 \mathrm{~Hz}, 1 \mathrm{H}, \mathrm{H}-5$ ), 2.81 (dq, $J=13.0,7.5 \mathrm{~Hz}, 1 \mathrm{H}, \mathrm{SEt}$ ), $2.76(\mathrm{dq}, J=13.0,7.5 \mathrm{~Hz}, 1 \mathrm{H}, \mathrm{SEt}), 1.27(\mathrm{dd}, J=7.5,7.5 \mathrm{~Hz}, 3 \mathrm{H}, \mathrm{SEt}) ;{ }^{13} \mathrm{C} \mathrm{NMR}\left(126 \mathrm{MHz}, \mathrm{CDCl}_{3}, 23.1^{\circ} \mathrm{C}\right) \delta$ 166.3 (s, G), 165.9 (s, G), 165.3 (s, G), 165.0 (s, G), 152.2 (s, 2C, G), 152.1 (s, 2C, G), 148.99 (s, 2C, G), 148.96 (s, 2C, G), 142.4 (s, G), 138.2 (s, G), 137.8 (s, G), 137.4 (s, G), 137.3 (s, Bn), 136.5 (s, 2C, Bn), 132.8 (d, 2C, Allyl), 132.6 (d, 2C, Allyl), 128.8-127.8 (overlapping 20 doublets and 1 singlets: 10 peaks were observed, $\mathrm{Bn}$ ), 124.7 (s, G), 124.0 (s, G), 123.9 (s, G), 123.5 (s, G), 117.7 (t, 4C, Allyl), 109.8 (d, 4C, G), 108.9 (d, 2C, G), 108.7 (d, 2C, G), 84.0 (d, C-1), 76.1 (d, C-5), 75.3 (t, 2C, Bn), 74.9 (t, Bn), 74.8 (t, Bn), 74.5 (d, C-3), 70.8 (d, C-2), 69.8 (t, 2C, Allyl), 69.7 (t, 2C, Allyl), 69.4 (d, C-4), 63.2 (t, C-6), 24.5 (t, SEt), 14.9 (q, SEt); HRMS (ESI) $\mathrm{m} / \mathrm{z}$ $[\mathrm{M}-\mathrm{H}]^{-}$calcd for $\mathrm{C}_{76} \mathrm{H}_{71} \mathrm{O}_{21} \mathrm{~S} 1351.4209$, found 1351.4226 .

\section{SI-07 Compound 24}
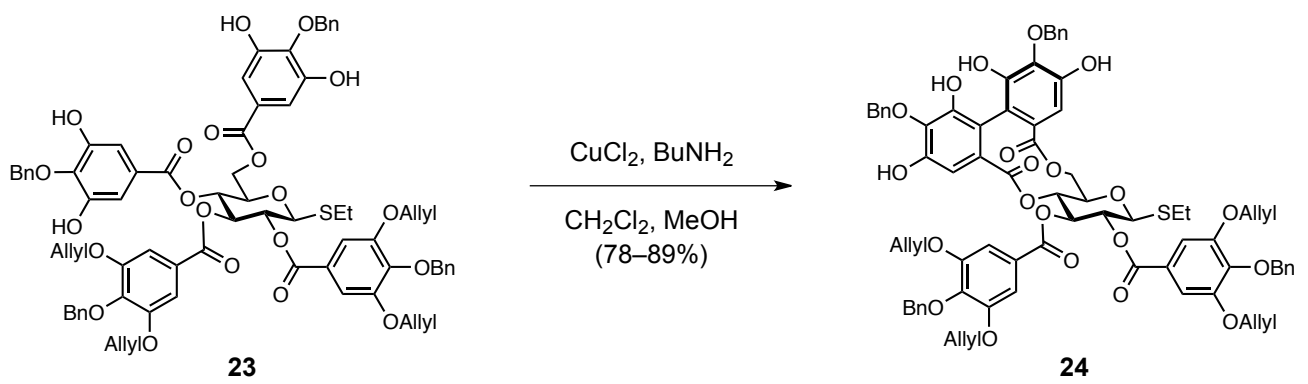

A solution of copper(II) chloride $(2.02 \mathrm{~g}, 15.0 \mathrm{mmol})$ and butylamine $(4.37 \mathrm{~g}, 60.0 \mathrm{mmol})$ in methanol $(75 \mathrm{~mL}$ ) was stirred for $30 \mathrm{~min}$ at $\mathrm{rt}$ to prepare a blue solution of copper(II) chloride butylamine complex. Subsequently, a solution of tetraol $23(4.06 \mathrm{~g}, 3.00 \mathrm{mmol})$ in dichloromethane $(75 \mathrm{~mL})$ was added to the solution of copper(II) chloride butylamine complex. The mixture was stirred for $1 \mathrm{~h}$ at $\mathrm{rt}$. The reaction mixture was diluted 
with ethyl acetate and to this was added sat. ammonium chloride aq. After extraction with ethyl acetate, the organic layer was successively washed with $1 \mathrm{M}$ hydrochloric acid, sat. sodium hydrogen carbonate aq, and brine. After the general drying procedure, the resulting residue was purified by CC (silica gel $300 \mathrm{~g}$, toluene/diethyl ether $=6 / 1$ to $2 / 1)$ to afford $24(3.26 \mathrm{~g}, 2.41 \mathrm{mmol}, 80 \%$ yield $)$ as a pale yellow amorphous solid: $[\alpha]_{\mathrm{D}}{ }^{24}+68(c$ $0.20, \mathrm{CHCl}_{3}$ ); IR (ATR) v 3495, 3088, 3065, 3030, 2959, 2940, 2880, 1749, 1724, 1717, 1585, 1497, 1456, 1433, $1423,1369,1331,1194,1128,1109,1028,752,735,698 \mathrm{~cm}^{-1} ;{ }^{1} \mathrm{H}$ NMR $\left(500 \mathrm{MHz}, \mathrm{CDCl}_{3}, 23.6^{\circ} \mathrm{C}\right) \delta 7.45(\mathrm{~d}, J$ $=7.0 \mathrm{~Hz}, 2 \mathrm{H}, \mathrm{Bn}), 7.41(\mathrm{~d}, J=7.0 \mathrm{~Hz}, 2 \mathrm{H}, \mathrm{Bn}), 7.37-7.23(\mathrm{~m}, 16 \mathrm{H}, \mathrm{Bn}), 7.18(\mathrm{~s}, 2 \mathrm{H}, \mathrm{G}), 7.12(\mathrm{~s}, 2 \mathrm{H}, \mathrm{G}), 6.74(\mathrm{~s}$, 1H, HHDP), 6.67 (s, 1H, HHDP), 5.82 (br s, 1H, OH), 5.67 (br s, 1H, OH), 5.61 (ddt, $J=17.0,10.5,5.0 \mathrm{~Hz}, 2 \mathrm{H}$, Allyl), 5.49 (ddt, $J=17.0,10.5,5.0 \mathrm{~Hz}, 2 \mathrm{H}$, Allyl), 5.61 (dd, $J=9.5,9.5 \mathrm{~Hz}, 1 \mathrm{H}, \mathrm{H}-3), 5.48$ (dd, $J=9.5,9.5 \mathrm{~Hz}$, 1H, H-2), 5.39 (ddt, $J=17.0,1.0,1.0 \mathrm{~Hz}, 2 \mathrm{H}$, Allyl), 5.38 (dd, $J=13.0,7.0 \mathrm{~Hz}, 1 \mathrm{H}, \mathrm{H}-6$ ), 5.33 (ddt, $J=17.0,1.0$, $1.0 \mathrm{~Hz}, 2 \mathrm{H}, \mathrm{Allyl}), 5.28$ (dd, $J=9.5,9.5 \mathrm{~Hz}, 1 \mathrm{H}, \mathrm{H}-4), 5.25$ (ddt, $J=10.5,1.0,1.0 \mathrm{~Hz}, 2 \mathrm{H}$, Allyl), 5.16 (ddt, $J=$ 10.5, 1.0, 1.0 Hz, 2H, Allyl), 5.14-5.10 (m, 4H, Bn), 5.07 (d, $J=9.0 \mathrm{~Hz}, 1 \mathrm{H}, \mathrm{Bn}), 5.06$ (d, $J=9.0 \mathrm{~Hz}, 1 \mathrm{H}, \mathrm{Bn})$, $5.01(\mathrm{~d}, J=9.5 \mathrm{~Hz}, 1 \mathrm{H}, \mathrm{Bn}), 4.99$ (d, $J=9.5 \mathrm{~Hz}, 1 \mathrm{H}, \mathrm{Bn}), 4.70$ (d, $J=9.5 \mathrm{~Hz}, 1 \mathrm{H}, \mathrm{H}-1), 4.54$ (dddd, $J=13.0,5.0$, 1.0, $1.0 \mathrm{~Hz}, 2 \mathrm{H}$, Allyl), 4.51 (dddd, $J=13.0,5.0,1.0,1.0 \mathrm{~Hz}, 2 \mathrm{H}$, Allyl), 4.47 (dddd, $J=13.0,5.0,1.0,1.0 \mathrm{~Hz}$, 2H, Allyl), 4.44 (dddd, $J=13.0,5.0,1.0,1.0 \mathrm{~Hz}, 2 \mathrm{H}$, Allyl), 4.07 (dd, $J=9.5,7.0 \mathrm{~Hz}, 1 \mathrm{H}, \mathrm{H}-5), 4.03$ (d, $J=13.0$ $\mathrm{Hz}, 1 \mathrm{H}, \mathrm{H}-6), 2.81$ (dq, $J=13.0,7.5 \mathrm{~Hz}, 1 \mathrm{H}, \mathrm{SEt}), 2.76$ (dq, $J=13.0,7.5 \mathrm{~Hz}, 1 \mathrm{H}, \mathrm{SEt}), 1.28$ (dd, $J=7.5,7.5 \mathrm{~Hz}$, $3 \mathrm{H}, \mathrm{SEt}) ;{ }^{13} \mathrm{C}$ NMR $\left(126 \mathrm{MHz}, \mathrm{CDCl}_{3}, 23.7^{\circ} \mathrm{C}\right) \delta 167.3$ (s, HHDP), 166.5 (s, HHDP), 165.8 (s, G), 164.9 (s, G), 152.3 (s, 2C, G), 152.1 (s, 2C, G), 149.03 (s, HHDP), 148.97 (s, HHDP), 147.1 (s, HHDP), 147.0 (s, HHDP), 142.5 (s, G), 142.3 (s, G), 137.43 (s, HHDP), 137.38 (s, HHDP), 136.3 (s, 2C, Bn), 135.8 (s, HHDP), 135.7 (s, HHDP), 132.8 (d, 4C, Allyl), 129.9 (s, Bn), 129.5 (s, Bn), 128.9-128.1 (overlapping 18 doublets: 9 peaks were observed, Bn), 127.9 (d, Bn), 127.8 (d, Bn), 123.9 (s, G), 123.6 (s, G), 117.8 (t, 2C, Allyl), 117.7 (t, 2C, Allyl), 113.7 (s, HHDP), 113.5 (s, HHDP), 109.0 (d, 2C, G), 108.9 (d, 2C, G), 108.5 (d, HHDP), 108.0 (d, HHDP), 83.9 (d, C-1), 75.9 (d, C-5), 75.6 (t, Bn), 75.5 (t, Bn), 74.9 (t, Bn), 74.8 (t, Bn), 74.6 (d, C-3), 70.5 (d, C-2), 70.2 (d, C4), 69.9 (t, 2C, Allyl), 69.7 (t, 2C, Allyl), 63.3 (t, C-6), 23.9 (t, SEt), 14.7 (q, SEt); HRMS (ESI) $\mathrm{m} / z$ [M - H] $]^{-}$ calcd for $\mathrm{C}_{76} \mathrm{H}_{69} \mathrm{O}_{21} \mathrm{~S} 1349.4052$, found 1349.4047 .

\section{SI-08 Compound 24 (demonstration of reproducibility)}

\section{Used $11.3 \mathrm{~g}$ of $\mathbf{2 3}$}

A solution of copper(II) chloride (5.61 g, $41.8 \mathrm{mmol})$ and butylamine (12.2 g, $167 \mathrm{mmol})$ in methanol $(167 \mathrm{~mL})$ was stirred for $50 \mathrm{~min}$ at $\mathrm{rt}$ to prepare a blue solution of copper(II) chloride butylamine complex. Subsequently, a solution of tetraol $23(11.3 \mathrm{~g}, 8.35 \mathrm{mmol})$ in dichloromethane $(167 \mathrm{~mL})$ was added to the solution of copper(II) chloride butylamine complex. The mixture was stirred for $1.8 \mathrm{~h}$ at rt. To the reaction mixture was added sat. ammonium chloride aq After extraction with diethyl ether, the organic layer was successively washed with $1 \mathrm{M}$ hydrochloric acid, sat. sodium hydrogen carbonate aq, and brine. After the general drying procedure, the resulting residue was purified by CC (silica gel $333 \mathrm{~g}$, toluene/diethyl ether =6/1 to 2/1) to afford $24(10.0 \mathrm{~g}, 7.40$ mmol, $89 \%$ yield).

\section{Used $20.8 \mathrm{~g}$ of $\mathbf{2 3}$}

A solution of copper(II) chloride $(10.3 \mathrm{~g}, 76.8 \mathrm{mmol})$ and butylamine $(22.4 \mathrm{~g}, 307 \mathrm{mmol})$ in methanol $(390 \mathrm{~mL})$ was stirred for $30 \mathrm{~min}$ at $\mathrm{rt}$ to prepare a blue solution of copper(II) chloride butylamine complex. Subsequently, a solution of tetraol $\mathbf{2 3}(20.8 \mathrm{~g}, 15.4 \mathrm{mmol})$ in dichloromethane $(390 \mathrm{~mL})$ was added to the solution of copper(II) chloride butylamine complex. The mixture was stirred for $1.5 \mathrm{~h}$ at rt. The reaction mixture was diluted with ethyl acetate and to this was added sat. ammonium chloride aq After extraction with ethyl acetate, the organic layer was successively washed with $1 \mathrm{M}$ hydrochloric acid, sat. sodium hydrogen carbonate aq, and brine. After the general drying procedure, the resulting residue was purified by CC (silica gel $600 \mathrm{~g}$, toluene/diethyl ether $=6 / 1$ to $2 / 1)$ to afford $\mathbf{2 4}(16.2 \mathrm{~g}, 12.0 \mathrm{mmol}, 78 \%$ yield $)$.

\section{SI-09 Compound 24 (without the use of dichloromethane as a solvent)}

A solution of copper(II) chloride (694 mg, $5.16 \mathrm{mmol})$ and butylamine $(3.77 \mathrm{~g}, 51.6 \mathrm{mmol})$ in methanol $(172 \mathrm{~mL})$ was stirred for $1 \mathrm{~h}$ at $\mathrm{rt}$ to prepare a blue solution of copper(II) chloride butylamine complex. Subsequently, a solution of tetraol $23(3.49 \mathrm{~g}, 2.58 \mathrm{mmol})$ in methanol $(86 \mathrm{~mL})$ was added to the solution of copper(II) 
chloride butylamine complex. After stirring for $50 \mathrm{~min}$ at $\mathrm{rt}$, to the reaction mixture was added copper(II) chloride $(694 \mathrm{mg}, 5.16 \mathrm{mmol})$ and butylamine $(3.77 \mathrm{~g}, 51.6 \mathrm{mmol})$. The mixture was stirred for $45 \mathrm{~min}$ at $\mathrm{rt}$. This was diluted with ethyl acetate and to this was added sat. ammonium chloride aq After extraction with ethyl acetate, the organic layer was successively washed with $1 \mathrm{M}$ hydrochloric acid, water, and brine. After the general drying procedure, the resulting residue was purified by CC (silica gel $50 \mathrm{~g}$, hexane/ethyl acetate $=1 / 1$ ) and further CC (silica gel $50 \mathrm{~g}$, toluene/diethyl ether $=9 / 1$ to 4/1) to afford 2/5 mixture of $\mathbf{2 4}$ and $\mathbf{2 3}$ (1.08 g, 24: $228 \mu \mathrm{mol}, 8.8 \%$ yield; 23: $570 \mu \mathrm{mol}, 22 \%$ yield, SI-33) and $\mathbf{2 4}(693 \mathrm{mg}, 513 \mu \mathrm{mol}, 20 \%$ yield). A part of the mixture of 24 and 23 was separated by PTLC ( solid. Note that the ${ }^{1} \mathrm{H}$ and ${ }^{13} \mathrm{C}$ NMR spectra of the reactant $\mathbf{2 3}$ and the recovered $\mathbf{2 3}$ were nonidentical (SI-34).

On the other hand, IR and HRMS data of the reactant $\mathbf{2 3}$ and the recovered $\mathbf{2 3}$ were superimposed. With the identical retention time of these two compounds, we finally concluded that these two are the same compounds. Data for 23 (recovered): $[\alpha]_{\mathrm{D}}{ }^{21}+23\left(c 0.60, \mathrm{CHCl}_{3}\right)$; IR (ATR) $v 3425,3030,2953,2872,1749,1724,1587,1452$, 1333, 1213, 1200, 1107, 997, $754 \mathrm{~cm}^{-1}$; ${ }^{1} \mathrm{H}$ NMR $\left(500 \mathrm{MHz}, \mathrm{CDCl}_{3}, 22.4{ }^{\circ} \mathrm{C}\right) \delta 7.45(\mathrm{~m}, 2 \mathrm{H}, \mathrm{Bn}), 7.41-7.23(\mathrm{~m}$, $18 \mathrm{H}, \mathrm{Bn}), 7.20(\mathrm{~s}, 2 \mathrm{H}, \mathrm{G}), 7.19$ (s, 2H, G), 7.06 (s, 2H, G), 7.05 (s, 2H, G), 6.08 (br s, 2H, OH), 6.02 (ddt, $J=$ 17.5, 10.5, 5.5 Hz, 2H, Allyl), 5.94 (ddt, $J=17.5,10.5,5.5 \mathrm{~Hz}, 2 \mathrm{H}$, Allyl), 5.79 (dd, $J=10.0,10.0 \mathrm{~Hz}, 1 \mathrm{H}, \mathrm{H}-3$ ), 5.57 (dd, $J=10.0,10.0 \mathrm{~Hz}, 1 \mathrm{H}, \mathrm{H}-4), 5.48$ (dd, $J=10.0,10.0 \mathrm{~Hz}, 1 \mathrm{H}, \mathrm{H}-2), 5.39$ (dd, $J=17.5,1.5 \mathrm{~Hz}, 2 \mathrm{H}$, Allyl), 5.32 (dd, $J=17.5,1.5 \mathrm{~Hz}, 2 \mathrm{H}$, Allyl), 5.25 (dd, $J=10.5,1.5 \mathrm{~Hz}, 2 \mathrm{H}$, Allyl), 5.16 (dd, $J=10.5,1.5 \mathrm{~Hz}, 2 \mathrm{H}$, Allyl), $5.14(\mathrm{~d}, J=11.5 \mathrm{~Hz}, 1 \mathrm{H}, \mathrm{Bn}), 5.12$ (d, $J=11.5 \mathrm{~Hz}, 1 \mathrm{H}, \mathrm{Bn}), 5.08$ (d, $J=11.5 \mathrm{~Hz}, 1 \mathrm{H}, \mathrm{Bn}), 5.06$ (s, 2H, Bn), 5.05 $(\mathrm{d}, J=11.5 \mathrm{~Hz}, 1 \mathrm{H}, \mathrm{Bn}), 5.00$ (d, $J=11.0 \mathrm{~Hz}, 1 \mathrm{H}, \mathrm{Bn}), 4.98$ (d, $J=11.0 \mathrm{~Hz}, 1 \mathrm{H}, \mathrm{Bn}), 4.82$ (d, $J=10.0 \mathrm{~Hz}, 1 \mathrm{H}$, $\mathrm{H}-1$ ), 4.55 (ddd, $J=5.5,1.5,1.5 \mathrm{~Hz}, 4 \mathrm{H}, \mathrm{Allyl}), 4.55-4.52$ (m, 2H, H-6), 4.46 (ddd, $J=5.5,1.5,1.5 \mathrm{~Hz}, 4 \mathrm{H}, \mathrm{Al}-$ lyl), 4.07 (m, 1H, H-5), 2.79 (dq, $J=14.5,7.5 \mathrm{~Hz}, 1 \mathrm{H}, \mathrm{SEt}), 2.74$ (dq, $J=14.5,7.5 \mathrm{~Hz}, 1 \mathrm{H}, \mathrm{SEt}), 1.27$ (dd, $J=$ 7.5, 7.5 Hz, 3H, SEt); ${ }^{13} \mathrm{C}$ NMR $\left(126 \mathrm{MHz}, \mathrm{CDCl}_{3}, 23.7^{\circ} \mathrm{C}\right) \delta 166.0(\mathrm{~s}, 2 \mathrm{C}, \mathrm{G}), 165.2(\mathrm{~s}, \mathrm{G}), 165.1(\mathrm{~s}, \mathrm{G}), 152.4$ (s, 2C, G), 152.3 (s, 2C, G), 149.1 (s, 2C, G), 142.6 (s, 2C, G), 142.6 (s, G), 138.3 (s, G), 137.9 (s, G), 137.6 (s, 1C, Bn), 137.5 (s, Bn), 136.6 (s, Bn), 136.6 (s, 2C, Bn), 133.0 (d, 2C, Allyl), 132.9 (d, 2C, Allyl), 129.0 (d, 6C, Bn), 128.7 (d, 2C, Bn), 128.6 (d, 2C, Bn), 128.5 (d, 2C, Bn), 128.4 (d, 2C, Bn), 128.3 (d, 2C, Bn), 128.3 (d, 2C, Bn), 128.1 (d, Bn), 128.1 (d, Bn), 125.3 (s, G), 124.4 (s, G), 124.1 (s, G), 123.8 (s, G), 118.0 (t, 4C, Allyl), 110.0 (d, 4C, G), 109.1 (d, 2C, G), 109.0 (d, 2C, G), 84.1 (d, H-1), 76.3 (d, H-5), 75.6 (t, 2C, Bn), 75.1 (t, Bn), 75.1 (t, Bn), 74.7 (d, H-3), 71.0 (d, H-2), 70.1 (t, 2C, Allyl), 70.0 (t, 2C, Allyl), 69.7 (d, H-4), 63.5 (t, H-6), 24.7 (t, SEt), 15.1 (q, SEt); HRMS (ESI) $m / z$ [M - H] $]^{-}$calcd for $\mathrm{C}_{76} \mathrm{H}_{71} \mathrm{O}_{21} \mathrm{~S} 1351.4209$, found 1351.4183.

\section{SI-10 Compound 31}
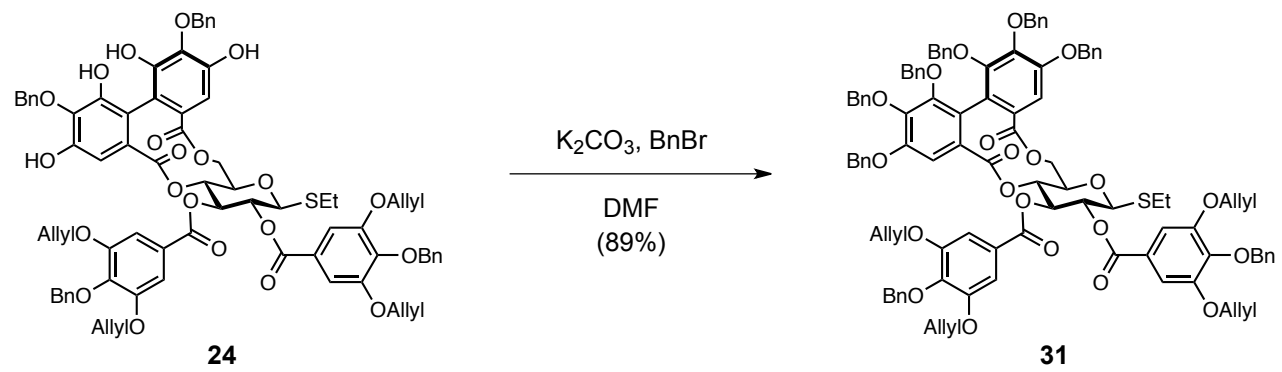

A mixture of tetraol $24(7.50 \mathrm{~g}, 5.55 \mathrm{mmol})$, potassium carbonate $(6.14 \mathrm{~g}, 44.4 \mathrm{mmol})$, and benzyl bromide $(7.59 \mathrm{~g}, 44.4 \mathrm{mmol})$ in DMF $(111 \mathrm{~mL})$ was stirred for $8 \mathrm{~h}$ at rt. The reaction was quenched with $1 \mathrm{M}$ hydrochloric acid at $0{ }^{\circ} \mathrm{C}$. From the mixture, the organic layer was separated and successively washed with $1 \mathrm{M}$ hydrochloric acid, water, and brine. After the general drying procedure, the resulting residue was purified by CC (silica gel $150 \mathrm{~g}$, hexane/ethyl acetate $=10 / 1$ to $4 / 1)$ to afford $31(8.45 \mathrm{~g}, 4.93 \mathrm{mmol}, 89 \%$ yield $)$ as a pale yellow amorphous solid: $[\alpha]_{\mathrm{D}}{ }^{25}+24\left(c 0.16, \mathrm{CHCl}_{3}\right)$; IR (ATR) $\vee 3030,2872,1748,1730,1585,1497,1454,1431,1420,1368$, 1329, 1182, 1094, 1009, 935, 910, 843, 750, 735, $696 \mathrm{~cm}^{-1} ;{ }^{1} \mathrm{H}$ NMR $\left(500 \mathrm{MHz}, \mathrm{CDCl}_{3}, 23.4{ }^{\circ} \mathrm{C}\right) \delta 7.49-7.20(\mathrm{~m}$, 29H, Bn), 7.20 (s, 2H, G), 7.14 (s, 2H, G), 7.14-7.10 (m, 3H, Bn), 7.01-6.93 (m, 5H, Bn), 6.98 (s, 1H, HHDP), 6.92 (d, $J=7.0 \mathrm{~Hz}, 2 \mathrm{H}, \mathrm{Bn}$ ), 6.86 (s, 1H, HHDP), 6.03 (ddt, $J=17.0,11.0,5.0 \mathrm{~Hz}, 2 \mathrm{H}$, Allyl), 5.88 (ddt, $J=17.0$, $11.0,5.0 \mathrm{~Hz}, 2 \mathrm{H}$, Allyl), 5.68 (dd, $J=10.0,10.0 \mathrm{~Hz}, 1 \mathrm{H}, \mathrm{H}-3), 5.50$ (dd, $J=10.0,10.0 \mathrm{~Hz}, 1 \mathrm{H}, \mathrm{H}-2), 5.41$ (ddt, $J$ $=17.0,1.0,1.0 \mathrm{~Hz}, 2 \mathrm{H}$, Allyl), 5.39 (dd, $J=13.5,6.5 \mathrm{~Hz}, 1 \mathrm{H}, \mathrm{H}-6), 5.35$ (dd, $J=10.0,10.0 \mathrm{~Hz}, 1 \mathrm{H}, \mathrm{H}-4), 5.28$ (ddt, $J=17.0,1.0,1.0 \mathrm{~Hz}, 2 \mathrm{H}$, Allyl), 5.27 (ddt, $J=11.0,1.0,1.0 \mathrm{~Hz}, 2 \mathrm{H}$, Allyl), 5.23 (d, $J=11.0 \mathrm{~Hz}, 1 \mathrm{H}, \mathrm{Bn}$ ), $5.15(\mathrm{~d}, J=11.0 \mathrm{~Hz}, 1 \mathrm{H}, \mathrm{Bn}), 5.12$ (ddt, $J=11.0,1.0,1.0 \mathrm{~Hz}, 2 \mathrm{H}, \mathrm{Allyl}), 5.08$ (s, 4H, Bn), 5.04 (d, $J=11.0 \mathrm{~Hz}$, 
1H, Bn), $5.01(\mathrm{~d}, J=11.0 \mathrm{~Hz}, 1 \mathrm{H}, \mathrm{Bn}), 5.00(\mathrm{~d}, J=11.0 \mathrm{~Hz}, 1 \mathrm{H}, \mathrm{Bn}), 4.95(\mathrm{~d}, J=11.0 \mathrm{~Hz}, 1 \mathrm{H}, \mathrm{Bn}), 4.94(\mathrm{~s}, 2 \mathrm{H}$, Bn), $4.94(\mathrm{~d}, J=11.0 \mathrm{~Hz}, 1 \mathrm{H}, \mathrm{Bn}), 4.90(\mathrm{~d}, J=11.0 \mathrm{~Hz}, 1 \mathrm{H}, \mathrm{Bn}), 4.84(\mathrm{~d}, J=11.0 \mathrm{~Hz}, 1 \mathrm{H}, \mathrm{Bn}), 4.78(\mathrm{~d}, J=11.0$ $\mathrm{Hz}, 1 \mathrm{H}, \mathrm{Bn}), 4.74$ (d, $J=10.5 \mathrm{~Hz}, 1 \mathrm{H}, \mathrm{Bn}), 4.74$ (d, $J=10.0 \mathrm{~Hz}, 1 \mathrm{H}, \mathrm{H}-1), 4.57$ (dddd, $J=12.5,5.0,1.0,1.0 \mathrm{~Hz}$, $2 \mathrm{H}$, Allyl), 4.55 (dddd, $J=12.5,5.0,1.0,1.0 \mathrm{~Hz}, 2 \mathrm{H}$, Allyl), 4.43 (dddd, $J=12.5,5.0,1.0,1.0 \mathrm{~Hz}, 2 \mathrm{H}$, Allyl), 4.40 (dddd, $J=12.5,5.0,1.0,1.0 \mathrm{~Hz}, 2 \mathrm{H}$, Allyl), 4.12 (dd, $J=10.0,6.5 \mathrm{~Hz}, 1 \mathrm{H}, \mathrm{H}-5), 4.09$ (d, $J=13.5 \mathrm{~Hz}, 1 \mathrm{H}$, H-6), 2.84 (dq, $J=12.5,7.5 \mathrm{~Hz}, 1 \mathrm{H}, \mathrm{SEt}), 2.80$ (dq, $J=12.5,7.5 \mathrm{~Hz}, 1 \mathrm{H}, \mathrm{SEt}), 1.30$ (dd, $J=7.5,7.5 \mathrm{~Hz}, 3 \mathrm{H}$, SEt); ${ }^{13} \mathrm{C}$ NMR $\left(126 \mathrm{MHz}, \mathrm{CDCl}_{3}, 23.7^{\circ} \mathrm{C}\right) \delta 167.4$ (s, HHDP), 166.7 (s, HHDP), 165.9 (s, G), 164.8 (s, G), 152.6 (s, HHDP), 152.5 (s, HHDP), 152.29 (s, 2C, G), 152.26 (s, HHDP), 152.20 (s, 2C, G), 152.17 (s, HHDP), 144.6 (s, HHDP), 144.4 (s, HHDP), 142.5 (s, G), 142.4 (s, G), 137.61 (s, HHDP), 137.59 (s, HHDP), 137.4 (s, 2C, Bn), 137.3 (s, Bn), 136.35 (s, Bn), 136.31 (s, Bn), 132.8 (d, 2C, Allyl), 132.6 (d, 2C, Allyl), 127.4-128.6 (overlapping 40 doublets and 3 singlets: 14 peaks were observed, Bn), 124.0 (s, G), 123.7 (s, G), 123.5 (s, HHDP), 123.3 (s, HHDP), 117.8 (t, 2C, Allyl), 117.7 (t, 2C, Allyl), 109.0 (d, 2C, G), 108.9 (d, 2C, G), 107.7 (d, 2C, HHDP), 84.1 (d, C-1), 76.3 (d, C-5), 75.5 (t, Bn), $75.4(\mathrm{t}, \mathrm{Bn}), 75.1(\mathrm{t}, \mathrm{Bn}), 74.91(\mathrm{t}, \mathrm{Bn}), 74.86(\mathrm{t}, \mathrm{Bn}), 74.8(\mathrm{t}$, Bn), 74.7 (d, C-3), 71.1 (t, Bn), 71.0 (t, Bn), 70.8 (d, C-2), 70.1 (d, C-4), 69.9 (t, 2C, Allyl), 69.7 (t, 2C, Allyl), 63.3 (t, C-6), 24.0 (t, SEt), 14.8 (q, SEt); HRMS (ESI) $m / z[\mathrm{M}+\mathrm{Na}]^{+}$calcd for $\mathrm{C}_{104} \mathrm{H}_{94} \mathrm{O}_{21} \mathrm{SNa}$ 1733.5906, found 1733.5907.

\section{SI-11 Compound 25}

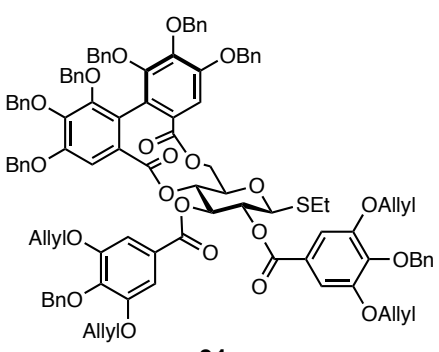

31

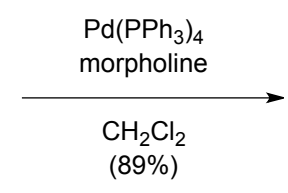

$\mathrm{Pd}\left(\mathrm{PPh}_{3}\right)_{4}(1.00 \mathrm{~g}, 865 \mu \mathrm{mol})$ was added to a solution of $\mathbf{3 1}(8.40 \mathrm{~g}, 4.91 \mathrm{mmol})$ and morpholine $(2.57 \mathrm{~g}$, $29.5 \mathrm{mmol})$ in dichloromethane $(100 \mathrm{~mL})$. The solution was stirred for $15 \mathrm{~h}$ at $\mathrm{rt}$. The reaction was quenched with sat. ammonium chloride aq. After extraction with ethyl acetate, the organic layer was successively washed with 1 $\mathrm{M}$ hydrochloric acid, water, and brine. After the general drying procedure, the crude product was purified by $\mathrm{CC}$ (silica gel $150 \mathrm{~g}$, hexane/ethyl acetate $=6 / 1$ to $3 / 2)$ to afford $\mathbf{2 5}(6.80 \mathrm{~g}, 4.38 \mathrm{mmol}, 89 \%$ yield) as a pale yellow amorphous solid: $[\alpha]_{\mathrm{D}}{ }^{25}+41\left(c 0.10, \mathrm{CHCl}_{3}\right)$; IR (ATR) v 3469, 3063, 3032, 2938, 2872, 1748, 1732, 1717, 1593, 1456, 1364, 1339, 1213, 1180, 1092, 1055, 1007, 908, 731, $696 \mathrm{~cm}^{-1} ;{ }^{1} \mathrm{H}$ NMR $\left(500 \mathrm{MHz}, \mathrm{CDCl}_{3}, 24.1^{\circ} \mathrm{C}\right) \delta$ 7.46 (br d, $J=8.0 \mathrm{~Hz}, 1 \mathrm{H}, \mathrm{Bn}), 7.43-7.37$ (m, 3H, Bn), 7.36-7.31 (m, 16H, Bn), 7.27-7.21 (m, 7H, Bn), 7.12 (s, 2H, G), 7.13-7.10 (m, 4H, Bn), 6.98 (s, 2H, G), 6.96 (s, 1H, HHDP), 6.89 (s, 1H, HHDP), 7.00-6.88 (m, 9H, Bn), 5.88 (br s, $1 \mathrm{H}, \mathrm{OH}), 5.76$ (br s, $1 \mathrm{H}, \mathrm{OH}), 5.63(\mathrm{dd}, J=10.0,10.0 \mathrm{~Hz}, 1 \mathrm{H}, \mathrm{H}-3), 5.50(\mathrm{dd}, J=10.0,10.0 \mathrm{~Hz}, 1 \mathrm{H}$, H-2), 5.38 (dd, $J=13.5,7.0 \mathrm{~Hz}, 1 \mathrm{H}, \mathrm{H}-6), 5.33$ (dd, $J=10.0,10.0 \mathrm{~Hz}, 1 \mathrm{H}, \mathrm{H}-4), 5.29$ (d, $J=11.5 \mathrm{~Hz}, 1 \mathrm{H}, \mathrm{Bn}$ ), $5.14(\mathrm{~d}, J=11.5 \mathrm{~Hz}, 1 \mathrm{H}, \mathrm{Bn}), 5.12$ (d, $J=11.5 \mathrm{~Hz}, 1 \mathrm{H}, \mathrm{Bn}), 5.09$ (d, $J=11.5 \mathrm{~Hz}, 1 \mathrm{H}, \mathrm{Bn}), 5.08$ (d, $J=11.5 \mathrm{~Hz}$, $1 \mathrm{H}, \mathrm{Bn}), 5.04$ (d, $J=11.5 \mathrm{~Hz}, 1 \mathrm{H}, \mathrm{Bn}), 5.01$ (d, $J=11.0 \mathrm{~Hz}, 1 \mathrm{H}, \mathrm{Bn}), 4.98$ (d, $J=11.0 \mathrm{~Hz}, 1 \mathrm{H}, \mathrm{Bn}), 4.96(\mathrm{~d}, J=$ $11.0 \mathrm{~Hz}, 1 \mathrm{H}, \mathrm{Bn}), 4.94$ (d, $J=11.0 \mathrm{~Hz}, 1 \mathrm{H}, \mathrm{Bn}), 4.94$ (d, $J=11.5 \mathrm{~Hz}, 1 \mathrm{H}, \mathrm{Bn}), 4.93$ (d, $J=11.0 \mathrm{~Hz}, 1 \mathrm{H}, \mathrm{Bn})$, $4.91(\mathrm{~d}, J=11.5 \mathrm{~Hz}, 1 \mathrm{H}, \mathrm{Bn}), 4.85(\mathrm{~d}, J=11.0 \mathrm{~Hz}, 1 \mathrm{H}, \mathrm{Bn}), 4.82(\mathrm{~d}, J=11.0 \mathrm{~Hz}, 1 \mathrm{H}, \mathrm{Bn}), 4.73(\mathrm{~d}, J=11.0 \mathrm{~Hz}$, $1 \mathrm{H}, \mathrm{Bn}), 4.71$ (d, $J=10.0 \mathrm{~Hz}, 1 \mathrm{H}, \mathrm{H}-1), 4.11(\mathrm{~m}, 1 \mathrm{H}, \mathrm{H}-5), 4.07$ (d, $J=13.5 \mathrm{~Hz}, 1 \mathrm{H}, \mathrm{H}-6), 2.82$ (dq, $J=13.0,7.5$ $\mathrm{Hz}, 1 \mathrm{H}, \mathrm{SEt}), 2.78$ (dq, $J=13.0,7.5 \mathrm{~Hz}, 1 \mathrm{H}, \mathrm{SEt}), 1.28$ (dd, $J=7.5,7.5 \mathrm{~Hz}, 3 \mathrm{H}, \mathrm{SEt}) ;{ }^{13} \mathrm{C}$ NMR $(126 \mathrm{MHz}$, $\left.\mathrm{CDCl}_{3}, 23.4^{\circ} \mathrm{C}\right) \delta 167.5$ (s, HHDP), 167.3 (s, HHDP), 165.7 (s, G), 165.1 (s, G), 152.6 (s, HHDP), 152.5 (s, HHDP), 152.2 (s, HHDP), 152.0 (s, HHDP), 149.0 (s, 2C, G), 148.7 (s, 2C, G), 144.43 (s, HHDP), 144.40 (s, HHDP), 138.1 (s, G), 137.9 (s, G), 137.6 (s, HHDP), 137.5 (s, HHDP), 137.4 (s, Bn), 137.3 (s, Bn), 136.51 (s, $\mathrm{Bn}$ ), 136.47 (s, Bn), 136.4 (s, Bn), 136.3 (s, Bn), 128.7-127.6 (overlapping 38 doublets and 2 singlets: 19 peaks were observed, Bn), 127.5 (d, Bn), 127.3 (d, Bn), 124.4 (s, G), 124.1 (s, G), 123.2 (s, 2C, HHDP), 109.9 (d, 2C, G), 109.8 (d, 2C, G), 108.0 (d, HHDP), 107.6 (d, HHDP), 84.1 (d, C-1), 75.7 (d, C-5), 75.41 (t, 2C, Bn), 75.38 (t, Bn), 75.2 (t, Bn), 75.1 (t, Bn), 75.0 (t, Bn), 74.8 (d, C-3), 71.0 (t, Bn), 70.8 (d, C-2), 70.7 (t, Bn), 70.6 (d, C-4), 63.3 (t, C-6), 24.3 (t, SEt), 14.8 (q, SEt); HRMS (ESI) $m / z$ [M - H] $]^{-}$calcd for $\mathrm{C}_{92} \mathrm{H}_{77} \mathrm{O}_{21} \mathrm{~S} 1549.4678$, found 1549.4666. 


\section{SI-12 Compound 26}

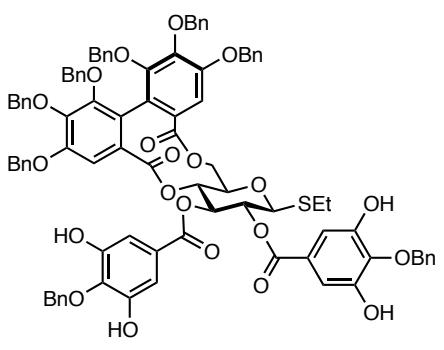

25

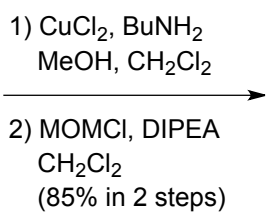
$(50 \mathrm{~mL})$ was stirred for $30 \mathrm{~min}$ at $\mathrm{rt}$ to prepare a blue solution of copper(II) chloride butylamine complex. Subsequently, a solution of tetraol $25(3.10 \mathrm{~g}, 2.00 \mathrm{mmol})$ in dichloromethane $(50 \mathrm{~mL})$ was added to the solution of copper(II) chloride butylamine complex. The mixture was stirred for $3.5 \mathrm{~h}$ at $\mathrm{rt}$. The reaction mixture was diluted with ethyl acetate and to this was added sat. ammonium chloride aq. After extraction with ethyl acetate, the organic layer was successively washed with $1 \mathrm{M}$ hydrochloric acid and brine. After the general drying procedure, the desired coupled product was roughly separated from the crude product by CC (silica gel $60 \mathrm{~g}$, hexane/ethyl acetate $=3 / 1$ to $2 / 3)$. A mixture of the roughly separated product, DIPEA ( $3.50 \mathrm{~g}, 27.1 \mathrm{mmol})$, and chloromethyl methyl ether $(1.09 \mathrm{~g}, 13.5 \mathrm{mmol})$ in dichloromethane $(33.8 \mathrm{~mL})$ was stirred for $15 \mathrm{~h}$ at $\mathrm{rt}$. To the reaction mixture were added sat. sodium hydrogen carbonate aq. After extraction with diethyl ether, the organic layer was washed with brine. After the general drying procedure, the resulting residue was purified by CC (silica gel $60 \mathrm{~g}$, hexane/ethyl acetate $=3 / 1$ to $2 / 1)$ to afford $26(2.94 \mathrm{~g}, 1.70 \mathrm{mmol}, 85 \%$ yield in 2 steps $)$ as a pale yellow amorphous solid: $[\alpha]_{\mathrm{D}}{ }^{25}-86\left(c 0.17, \mathrm{CHCl}_{3}\right)$; IR (ATR) v 3030, 2934, 1749, 1456, 1369, 1329, 1171, 1096, 1042, 1009, 910 , 737, $696 \mathrm{~cm}^{-1}$; ${ }^{1} \mathrm{H}$ NMR $\left(500 \mathrm{MHz}, \mathrm{CDCl}_{3}, 23.7^{\circ} \mathrm{C}\right) \delta 7.51-7.23(\mathrm{~m}, 29 \mathrm{H}, \mathrm{Bn}), 7.14-7.09(\mathrm{~m}, 7 \mathrm{H}, \mathrm{Bn}), 7.02-$ 6.99 (m, 4H, Bn), 7.00 (s, 1H, HHDP), 6.98 (s, 1H, HHDP), 6.94 (s, 1H, HHDP), 6.85 (s, 1H, HHDP), 5.36 (dd, $J$ $=13.0,6.5 \mathrm{~Hz}, 1 \mathrm{H}, \mathrm{H}-6), 5.31(\mathrm{dd}, J=10.0,9.0 \mathrm{~Hz}, 1 \mathrm{H}, \mathrm{H}-3), 5.26(\mathrm{~d}, J=11.0 \mathrm{~Hz}, 1 \mathrm{H}, \mathrm{Bn}), 5.23(\mathrm{~d}, J=10.0 \mathrm{~Hz}$, $1 \mathrm{H}, \mathrm{MOM}), 5.21(\mathrm{dd}, J=10.0,10.0 \mathrm{~Hz}, 1 \mathrm{H}, \mathrm{H}-4), 5.19(\mathrm{~d}, J=10.0 \mathrm{~Hz}, 1 \mathrm{H}, \mathrm{Bn}), 5.16-5.05(\mathrm{~m}, 8 \mathrm{H}, \mathrm{Bn}$ and MOM), 5.12 (dd, $J=10.0,9.0 \mathrm{~Hz}, 1 \mathrm{H}, \mathrm{H}-2), 5.02-4.95$ (m, 6H, Bn and MOM), 4.91 (d, $J=10.0 \mathrm{~Hz}, 1 \mathrm{H}, \mathrm{Bn})$, 4.89 (d, $J=10.5 \mathrm{~Hz}, 1 \mathrm{H}, \mathrm{Bn}), 4.82-4.72(\mathrm{~m}, 5 \mathrm{H}, \mathrm{Bn}$ and MOM), 4.66 (d, $J=10.0 \mathrm{~Hz}, 1 \mathrm{H}, \mathrm{H}-1), 4.05(\mathrm{~d}, J=13.0$ Hz, 1H, H-6), 3.99 (dd, $J=10.0,6.5$ Hz, 1H, H-5), 3.45 (s, 3H, MOM), 3.042 (s, 3H, MOM), 3.038 (s, 3H, MOM), 2.99 (s, 3H, MOM), 2.83 (dq, $J=13.0,7.5 \mathrm{~Hz}, 1 \mathrm{H}, \mathrm{SEt}), 2.77$ (dq, $J=13.0,7.5 \mathrm{~Hz}, 1 \mathrm{H}, \mathrm{SEt}), 1.33$ (dd, $J$ $=7.5,7.5 \mathrm{~Hz}, 3 \mathrm{H}, \mathrm{SEt}) ;{ }^{13} \mathrm{C}$ NMR $\left(126 \mathrm{MHz}, \mathrm{CDCl}_{3}, 23.4{ }^{\circ} \mathrm{C}\right) \delta 168.1$ (s, HHDP), 167.2 (s, HHDP), 166.8 (s, HHDP), 166.7 (s, HHDP), 152.62 (s, HHDP), 152.56 (s, HHDP), 152.3 (s, HHDP), 152.2 (s, HHDP), 151.0 (s, HHDP), 150.9 (s, HHDP), 150.3 (s, HHDP), 149.8 (s, HHDP), 144.7 (s, HHDP), 144.6 (s, HHDP), 143.5 (s, HHDP), 143.4 (s, HHDP), 137.75 (s, HHDP), 137.71 (s, HHDP), 137.5 (s, HHDP), 137.4 (s, HHDP), 137.13 (s, $\mathrm{Bn}), 137.10$ (s, Bn), 136.4 (s, 2C, Bn), 129.0 (s, Bn), 128.7 (s, Bn), 128.4-127.4 (overlapping 40 doublets and 2 singlets: 15 peaks were observed, Bn), 123.9 (s, HHDP), 123.6 (s, HHDP), 122.5 (s, HHDP), 122.1 (s, HHDP), 110.0 (d, HHDP), 109.4 (d, HHDP), 108.1 (d, HHDP), 107.4 (d, HHDP), 99.4 (t, MOM), 99.3 (t, MOM), 95.3 (t, MOM), 95.1 (t, MOM), 82.3 (d, C-1), 77.9 (d, C-3), 76.5 (d, C-5), 75.54 (t, Bn), 75.48 (t, Bn), 75.1 (t, Bn), 75.0 (t, 2C, Bn), 74.9 (t, Bn), 74.3 (d, C-2), 71.2 (t, Bn), 71.1 (t, Bn), 69.0 (d, C-4), 63.2 (t, C-6), 56.9 (q, MOM), 56.8 (q, MOM), 56.4 (q, MOM), 55.9 (q, MOM), 23.5 (t, SEt), 14.9 (q, SEt); HRMS (ESI) $\mathrm{m} / z$ [M + Na] calcd for $\mathrm{C}_{100} \mathrm{H}_{92} \mathrm{O}_{25} \mathrm{SNa}$ 1747.5546, found 1747.5539. 


\section{SI-13 Compound 27}

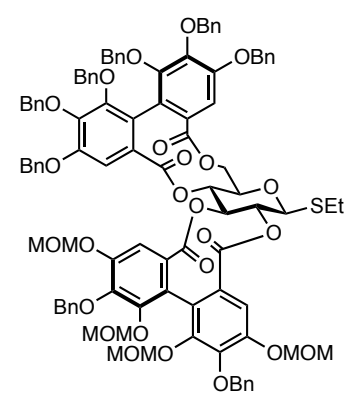

26

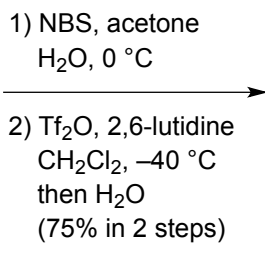
$\mathrm{mL}$ ) was stirred for $45 \mathrm{~min}$ at $0{ }^{\circ} \mathrm{C}$. After to sat. sodium hydrogen carbonate aq and $10 \%$ sodium thiosulfate aq was added the reaction mixture, acetone was removed by rotary evaporation. The remaining aqueous mixture was extracted with ethyl acetate. The organic layer was successively washed with $1 \mathrm{M}$ hydrochloric acid, sat. sodium hydrogen carbonate aq, and brine. After the general drying procedure, the resulting residue was roughly separated by $\mathrm{CC}$ (silica gel $165 \mathrm{~g}$, hexane/ethyl acetate $=5 / 1$ to $1 / 3$ ) to obtain the corresponding sulfoxides as a 50/50 mixture of the diastereomers (SI-35). To a mixture of the roughly separated sulfoxides and 2,6-lutidine (1.25 g, 11.7 $\mathrm{mmol})$ in dichloromethane $(117 \mathrm{~mL})$ was added $\mathrm{Tf}_{2} \mathrm{O}(1.65 \mathrm{~g}, 5.86 \mathrm{mmol})$ at $-40^{\circ} \mathrm{C}$. The mixture was stirred for $50 \mathrm{~min}$ at $-40^{\circ} \mathrm{C}$. After addition of sat. sodium hydrogen carbonate aq, the mixture was extracted with ethyl acetate. The organic layer was successively washed with $1 \mathrm{M}$ hydrochloric acid, sat. sodium hydrogen carbonate aq, and brine. After the general drying procedure, the crude product was purified by CC (silica gel $45 \mathrm{~g}$, hexane/ethyl acetate $=3 / 1$ to $1 / 1)$ to afford $27(3.88 \mathrm{~g}, 2.31 \mathrm{mmol}, 75 \%$ yield in 2 steps) as a mixture of anomers: IR (ATR) $v$ $3462,3065,3030,2953,1748,1728,1591,1329,1186,1153,1096,1045,752,696 \mathrm{~cm}^{-1}$; HRMS (ESI) $m / z$ [M + $\mathrm{Na}]^{+}$calcd for $\mathrm{C}_{98} \mathrm{H}_{88} \mathrm{O}_{26} \mathrm{Na} 1703.5462$, found 1703.5459 .

\section{SI-14 Compound 27 (Unsuccess conditions)}
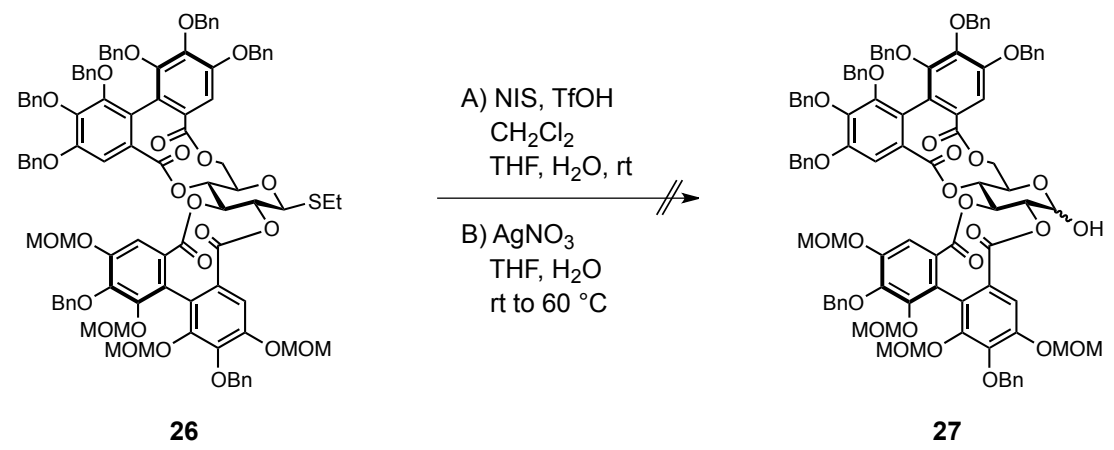

Method A

A solution of $26(10.4 \mathrm{mg}, 5.00 \mu \mathrm{mol})$, NIS ( $3.4 \mathrm{mg}, 15 \mu \mathrm{mol})$, and triflic acid $(0.5 \mathrm{mg}, 3 \mu \mathrm{mol})$ in dichloromethane $(0.2 \mathrm{~mL})$, tetrahydrofuran $(0.2 \mathrm{~mL})$, and water $(0.1 \mathrm{~mL})$ was stirred for $4 \mathrm{~h}$ at $\mathrm{rt}$. After stirring for $3 \mathrm{~h}$ at $\mathrm{rt}$ with further addition of NIS and $\mathrm{TfOH}$, the starting material decomposed.

\section{Method B}

A solution of $26(10.4 \mathrm{mg}, 5.00 \mu \mathrm{mol})$ and silver(I) nitrate $(3.4 \mathrm{mg}, 20 \mu \mathrm{mol})$ in THF $(0.4 \mathrm{~mL})$ and water $(0.1 \mathrm{~mL})$ was stirred for $50 \mathrm{~min}$ at $\mathrm{rt}$. The mixture was further stirred for $25 \mathrm{~h}$ at $60^{\circ} \mathrm{C}$. No reaction occurred. 


\section{SI-15 Compound 28}
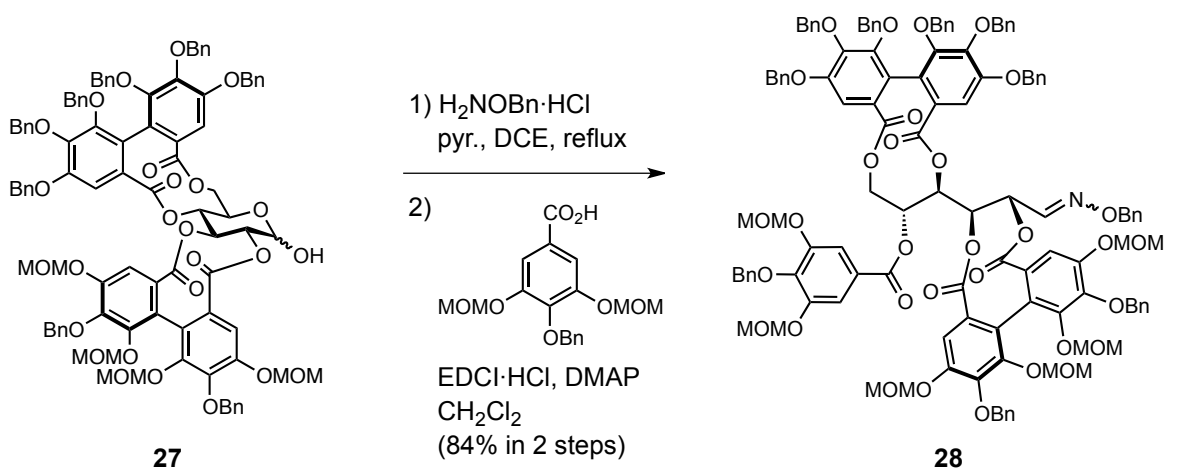

A solution of 27 (767 mg, $456 \mu \mathrm{mol}), \mathrm{H}_{2} \mathrm{NOBn} \cdot \mathrm{HCl}(291 \mathrm{mg}, 1.82 \mathrm{mmol})$, and pyridine (289 mg, 3.65 $\mathrm{mmol})$ in DCE $(22.3 \mathrm{~mL})$ was stirred for $3 \mathrm{~h}$ at reflux. After addition of sat. ammonium chloride aq at $\mathrm{rt}$, the mixture was extracted with ethyl acetate. The organic layer was successively washed with $1 \mathrm{M}$ hydrochloric acid, sat. sodium hydrogen carbonate aq, and brine. After the general drying procedure, the obtained crude product was used for the subsequent transformation without further purification. A mixture of the crude product, $\mathrm{Bn} / \mathrm{MOM}$ protected gallic acid ${ }^{26}(191 \mathrm{mg}, 547 \mu \mathrm{mol})$, DMAP $(55.7 \mathrm{mg}, 456 \mu \mathrm{mol})$, and EDCI hydrochloride $(349 \mathrm{mg}, 1.82$ $\mathrm{mmol})$ in dichloromethane $(22.3 \mathrm{~mL})$ was stirred for $20.5 \mathrm{~h}$ at rt. After removal of dichloromethane by rotary evaporation, to the residue was added diethyl ether and sat. sodium hydrogen carbonate aq. The aqueous mixture was extracted with diethyl ether. The organic layer was washed with brine. After the general drying procedure, the crude product was purified by CC (silica gel $30 \mathrm{~g}$, hexane/ethyl acetate $=4 / 1$ to $1 / 1$ ) to afford $\mathbf{2 8}[807 \mathrm{mg}$, a 92/8 mixture of oximes (SI-36), $381 \mu \mathrm{mol}, 84 \%$ yield in 2 steps] as a colorless amorphous solid: Data for the major isomer of 28: $[\alpha]_{\mathrm{D}}{ }^{24}-64\left(c 0.21, \mathrm{CHCl}_{3}\right)$; IR (ATR) $v$ 3064, 3032, 2953, 1751, 1717, 1591, 1497, 1481, 1454, 1368, 1327, 1171, 1153, 1096, 1043, 1001, 984, 910, 735, $696 \mathrm{~cm}^{-1} ;{ }^{1} \mathrm{H}$ NMR $\left(500 \mathrm{MHz}, \mathrm{CDCl}_{3}, 23.9^{\circ} \mathrm{C}\right) \delta 7.51$ (s, 2H, G), 7.50 (d, $J=6.0 \mathrm{~Hz}, 1 \mathrm{H}, \mathrm{H}-1), 7.50-7.22(\mathrm{~m}, 33 \mathrm{H}, \mathrm{Bn}), 7.18-7.16$ (m, 5H, Bn), 7.15 (s, 1H, HHDP), 7.13-7.08 (m, 4H, Bn), 7.12 (s, 1H, HHDP), 7.06-7.03 (m, 3H, Bn), 7.01 (s, 1H, HHDP), 6.98-6.92 (m, 5H, Bn), $6.96(\mathrm{~s}, 1 \mathrm{H}, \mathrm{HHDP}), 5.90$ (dd, $J=9.5,6.0 \mathrm{~Hz}, 1 \mathrm{H}, \mathrm{H}-2), 5.77$ (dd, $J=9.5,1.0 \mathrm{~Hz}, 1 \mathrm{H}, \mathrm{H}-3), 5.67$ (dd, $J=7.0,1.0$ $\mathrm{Hz}, 1 \mathrm{H}, \mathrm{H}-4), 5.57$ (m, 1H, H-5), 5.25 (d, $J=11.5 \mathrm{~Hz}, 1 \mathrm{H}, \mathrm{Bn}), 5.21(\mathrm{~d}, J=11.0 \mathrm{~Hz}, 1 \mathrm{H}, \mathrm{Bn}), 5.18-5.16(\mathrm{~m}, 4 \mathrm{H}$, MOM), 5.14-5.10 (m, 6H, Bn and MOM), $5.08(\mathrm{~d}, J=6.0 \mathrm{~Hz}, 1 \mathrm{H}, \mathrm{MOM}), 5.06(\mathrm{~s}, 2 \mathrm{H}, \mathrm{Bn}), 5.04(\mathrm{~d}, J=6.0 \mathrm{~Hz}$, $1 \mathrm{H}, \mathrm{MOM}), 5.03$ (d, $J=6.0 \mathrm{~Hz}, 1 \mathrm{H}, \mathrm{MOM}), 5.00-4.97$ (m, 7H, Bn and MOM), 4.91-4.87 (m, 5H, Bn, MOM, and H-6), $4.81(\mathrm{~d}, J=11.0 \mathrm{~Hz}, 1 \mathrm{H}, \mathrm{Bn}), 4.77(\mathrm{~d}, J=6.0 \mathrm{~Hz}, 1 \mathrm{H}, \mathrm{MOM}), 4.70(\mathrm{~d}, J=11.0 \mathrm{~Hz}, 1 \mathrm{H}, \mathrm{Bn}), 4.67(\mathrm{~d}, J$ $=5.5 \mathrm{~Hz}, 1 \mathrm{H}, \mathrm{MOM}), 4.39(\mathrm{~d}, J=12.0 \mathrm{~Hz}, 1 \mathrm{H}, \mathrm{H}-6), 3.41(\mathrm{~s}, 3 \mathrm{H}, \mathrm{MOM}), 3.40(\mathrm{~s}, 6 \mathrm{H}, \mathrm{MOM}), 3.77(\mathrm{~s}, 3 \mathrm{H}$, MOM), 3.07 (s, 3H, MOM), 2.97 (s, 3H, MOM); $\left.{ }^{13} \mathrm{C} \mathrm{NMR} \mathrm{(126} \mathrm{MHz,} \mathrm{CDCl}_{3}, 24.0{ }^{\circ} \mathrm{C}\right) \delta 167.7$ (s, HHDP), 167.0 (s, HHDP), 166.5 (s, HHDP), 166.1 (s, HHDP), 165.0 (s, G), 152.59 (s, HHDP), 152.56 (s, HHDP), 152.4 (s, HHDP), 152.2 (s, HHDP), 151.2 (s, 2C, G), 150.9 (s, 2C, HHDP), 150.16 (s, HHDP), 150.20 (s, HHDP), 144.8 (s, HHDP), 144.3 (s, HHDP), 144.0 (s, G), 143.5 (s, HHDP), 143.2 (s, HHDP), 142.4 (d, C-1), 137.7 (s, Bn), 137.64 (s, HHDP), 137.57 (s, Bn), 137.4 (s, HHDP), 137.22 (s, Bn), 137.18 (s, HHDP), 136.5 (s, Bn), 136.3 (s, Bn), 136.2 (s, HHDP), 129.10 (s, Bn), 129.05 (s, Bn), 128.9 (s, Bn), 128.5-127.4 (overlapping 50 doublets and 2 singlets: 19 peaks were observed, Bn), 124.3 (s, G), 123.5 (s, HHDP), 122.8 (s, HHDP), 121.9 (s, HHDP), 121.6 (s, HHDP), 112.8 (d, 2C, G), 110.2 (d, HHDP), 109.3 (d, HHDP), 108.3 (d, HHDP), 107.8 (d, HHDP), 99.5 (t, MOM), 99.4 (t, MOM), 95.7 (t, MOM), 95.5 (t, 2C, MOM), 95.3 (t, MOM), 76.9 (t, Bn), 75.4 (t, 2C, Bn), 75.2 (t, Bn), 75.15 (d, C-3), 75.11 (t, Bn), 75.04 (t, 2C, Bn), 74.99 (t, Bn), 71.4 (d, C-2), 71.2 (d, C-5), 71.0 (t, Bn), 70.9 (t, Bn), 70.0 (d, C-4), 63.6 (t, C-6), 57.0 (q, MOM), 56.8 (q, MOM), 56.44 (q, 2C, MOM), 56.40 (q, MOM), 56.2 (q, MOM); HRMS (ESI) $m / z[\mathrm{M}+\mathrm{Na}]^{+}$calcd for $\mathrm{C}_{123} \mathrm{H}_{113} \mathrm{NNaO}_{32} 2138.7143$, found 2138.7164 . 


\section{SI-16 Compound 29}
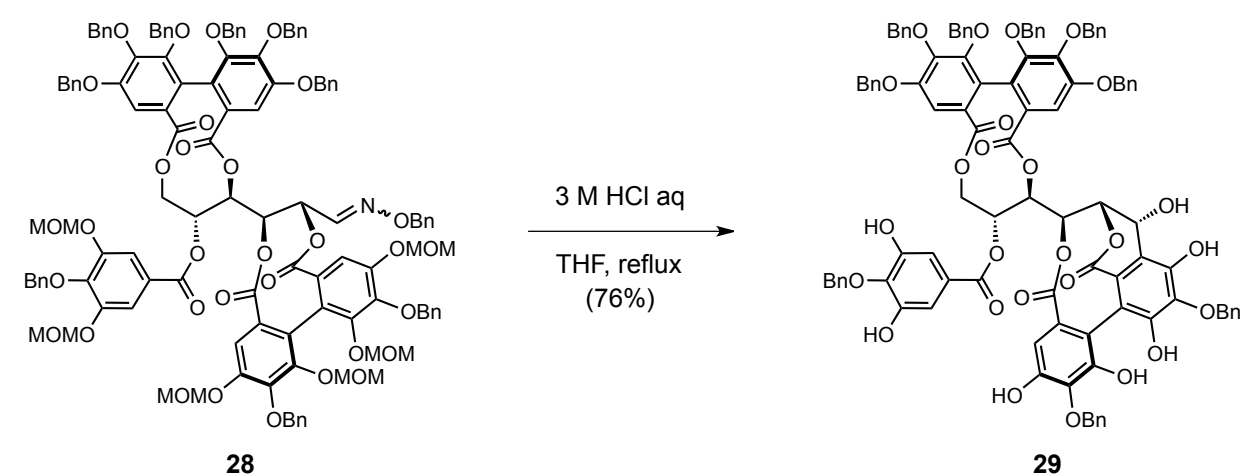

A solution of $\mathbf{2 8}(161 \mathrm{mg}, 75.8 \mu \mathrm{mol})$ in THF $(3.8 \mathrm{~mL})$ and $3 \mathrm{M}$ hydrochloric acid $(152 \mu \mathrm{L})$ was stirred for $3 \mathrm{~h}$ at reflux. After cooling to $\mathrm{rt}$ and addition of water, the mixture was extracted with ethyl acetate. The organic layer was washed with brine. After the general drying procedure, the crude product was purified by CC (silica gel $16 \mathrm{~g}$, hexane/ethyl acetate $=13 / 10$ to $2 / 3)$ to afford $\mathbf{2 9}(100 \mathrm{mg}, 57.2 \mu \mathrm{mol}, 76 \%$ yield $)$ as a colorless amorphous solid: $[\alpha]_{\mathrm{D}}{ }^{25}-14\left(c 0.20, \mathrm{CHCl}_{3}\right)$; IR (ATR) $v 3472,3061,3034,2941,2870,1744,1722,1591,1454$, 1362, 1329, 1171, 1092, 1063, 968, 910, 741, $694 \mathrm{~cm}^{-1}$; ${ }^{1} \mathrm{H}$ NMR $\left(500 \mathrm{MHz}, \mathrm{CDCl}_{3}, 22.9^{\circ} \mathrm{C}\right) \delta 7.34-7.15(\mathrm{~m}$, 34H, Bn and HHDP), 7.14 (s, 2H, G), 7.07-6.99 (m, 8H, Bn), 6.93 (d, $J=7.5 \mathrm{~Hz}, 2 \mathrm{H}, \mathrm{Bn}), 6.88(\mathrm{~d}, J=7.5 \mathrm{~Hz}$, 2H, Bn), 6.85 (s, 1H, HHDP), 6.39 (s, 1H, HHDP), 6.29 (br s, 2H, OH), 6.13 (br s, 1H, OH), 5.73 (d, $J=8.5 \mathrm{~Hz}$, 1H, H-4), 5.70-5.63 (m, 2H, H-3 and H-5), 5.43 (d, $J=5.5 \mathrm{~Hz}, 1 \mathrm{H}, \mathrm{H}-1), 5.02-4.82$ (m, 19H, Bn, H-2, and H-6), $4.59(\mathrm{~d}, J=11.0 \mathrm{~Hz}, 1 \mathrm{H}, \mathrm{Bn}), 4.13($ br s, $1 \mathrm{H}, \mathrm{OH}), 4.12$ (d, $J=13.5 \mathrm{~Hz}, 1 \mathrm{H}, \mathrm{H}-6) ;{ }^{13} \mathrm{C}$ NMR $\left(126 \mathrm{MHz}, \mathrm{CDCl}_{3}\right.$, $23.9^{\circ} \mathrm{C}$ ) $\delta 170.0$ (s, HHDP), 168.1 (s, HHDP), 167.6 (s, HHDP), 165.8 (s, G), 164.9 (s, HHDP), 152.6 (s, HHDP), 152.5 (s, HHDP), 152.25 (s, HHDP), 152.17 (s, HHDP), 149.7 (s, HHDP), 149.1 (s, 2C, G), 148.8 (s, HHDP), 146.8 (s, HHDP), 146.1 (s, HHDP), 144.9 (s, HHDP), 143.8 (s, HHDP), 138.4 (s, HHDP), 137.9 (s, G), 137.8 (s, HHDP), 137.5 (s, HHDP), 137.4 (s, Bn), 137.1 (s, HHDP), 136.7 (s, Bn), 136.5 (s, Bn), 136.34 (s, Bn), 136.28 (s, G), 136.0 (s, HHDP), 134.5 (s, HHDP), 130.2 (s, Bn), 129.4 (s, Bn), 128.8-127.8 (overlapping 43 doublets: 12 peaks were observed, Bn), 127.45 (s, Bn), 127.37 (s, Bn), 127.3 (s, Bn), 124.3 (d, Bn), 124.2 (d, Bn), 123.2 (s, HHDP), 122.4 (s, HHDP), 118.1 (s, HHDP), 115.3 (s, HHDP), 113.3 (s, HHDP), 110.0 (d, 2C, G), 108.7 (d, HHDP), 106.9 (d, HHDP), 106.4 (d, HHDP), 75.62 (t, Bn), 75.55 (t, 2C, Bn), 75.5 (t, Bn), 75.3 (d, C-2), 75.2 (t, Bn), 75.0 (t, Bn), 74.9 (t, Bn), 73.4 (d, C-4), 70.9 (t, Bn), 70.7 (t, Bn), 69.7 (d, C-5), 69.5 (d, C-3), 65.5 (d, C-1), 65.1 (t, C-6); HRMS (ESI) $m / z[\mathrm{M}-\mathrm{H}]^{-}$calcd for $\mathrm{C}_{104} \mathrm{H}_{81} \mathrm{O}_{26}$ 1745.5016, found 1745.5038.

\section{SI-17 Compound 29 (neutralized at workup)}

\section{The experiment resulted in 50\% yield}

A solution of $\mathbf{2 8}(65.8 \mathrm{mg}, 31.1 \mu \mathrm{mol})$ in THF $(1.6 \mathrm{~mL})$ and $3 \mathrm{M}$ hydrochloric acid $(60 \mu \mathrm{L})$ was stirred for $1 \mathrm{~h}$ at $60{ }^{\circ} \mathrm{C}$ and for $3 \mathrm{~h}$ at reflux. After cooling to $\mathrm{rt}$ and addition of sat. sodium hydrogen carbonate aq (pH of the water layer $=10$ by $\mathrm{pH}$-test paper), the mixture was extracted with ethyl acetate. The organic layer was washed with brine. After the general drying procedure, the crude product was purified by CC (silica gel $2.5 \mathrm{~g}$, hexane/ethyl acetate $=1 / 2$ to $2 / 3)$ to afford $29(27.2 \mathrm{mg}, 15.6 \mu \mathrm{mol}, 50 \%$ yield $)$.

The experiment resulted in $36 \%$ yield

A solution of $28(683 \mathrm{mg}, 326 \mu \mathrm{mol})$ in THF $(16 \mathrm{~mL})$ and $3 \mathrm{M}$ hydrochloric acid $(0.7 \mathrm{~mL})$ was stirred for $9 \mathrm{~h}$ at reflux. After cooling to $\mathrm{rt}$ and addition of sat. sodium hydrogen carbonate aq $(\mathrm{pH}$ of the water layer $=10$ by $\mathrm{pH}$-test paper), the mixture was extracted with ethyl acetate. The organic layer was washed with brine. After the general drying procedure, the crude product was purified by $\mathrm{CC}$ ( ilica gel $20 \mathrm{~g}$, hexane/ethyl acetate $=1 / 2$ to $2 / 3$ ) to afford 29 (204 mg, $118 \mu \mathrm{mol}, 36 \%$ yield).

In these two cases, hydrolysis of the 5-O-galloyl moiety of 29 was indicated by mass spectrometry $(\mathrm{m} / \mathrm{z}$ 1504.6353, SI-37). 


\section{SI-18 Casuarinin (1)}
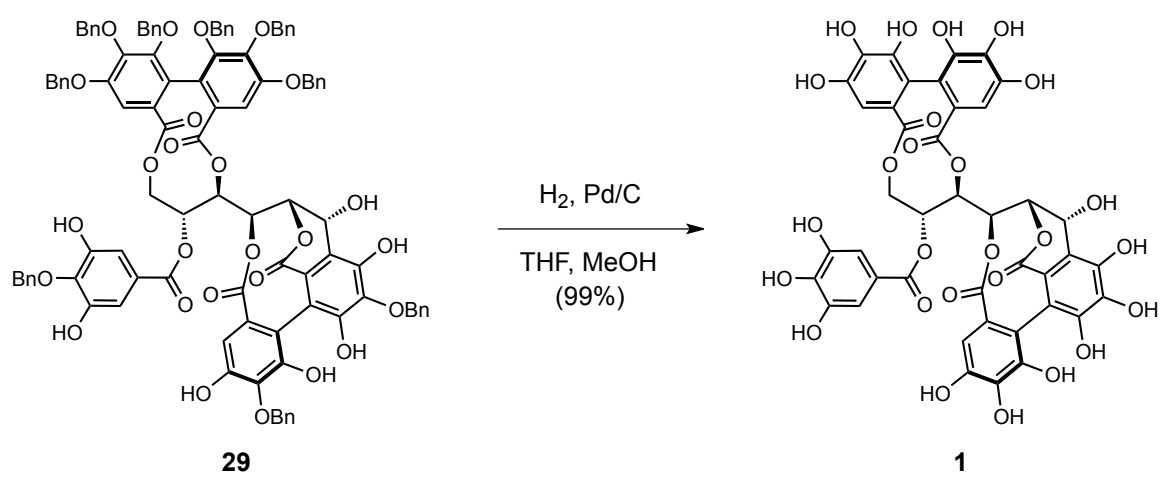

A mixture of 29 (396 mg, $227 \mu \mathrm{mol})$ and palladium on carbon (wetted with ca. 55\% water, $10 \mathrm{wt}$. $\%$ on carbon, $5.4 \mathrm{mg} ; 0.24 \mathrm{mg}$ as $\mathrm{Pd}, 2.3 \mu \mathrm{mol})$ in methanol $(3.0 \mathrm{~mL})$ and THF $(3.0 \mathrm{~mL})$ was stirred for $12 \mathrm{~h}$ at $\mathrm{rt}$ under $\mathrm{H}_{2}$ atmosphere. The mixture was filtered through a cotton-Celite pad to remove the catalyst and carbon. The crude product was purified by CC (Sephadex LH-20 $14 \mathrm{~g}$, methanol) to afford casuarinin (1) (211 mg, $225 \mu \mathrm{mol}$, $99 \%$ yield) as a colorless amorphous solid: $[\alpha]_{\mathrm{D}}^{22}+42(c 0.48, \mathrm{MeOH})$; IR (ATR) $v 3395,2980,2924,2857,1738$, $1732,1614,1516,1454,1352,1319,1182,1090,1042,868,764,737,650 \mathrm{~cm}^{-1}$; HRMS (ESI) $\mathrm{m} / z$ [M - H] calcd for $\mathrm{C}_{41} \mathrm{H}_{27} \mathrm{O}_{26}$ 935.0791, found 935.0788; ${ }^{1} \mathrm{H}$ NMR and ${ }^{13} \mathrm{C}$ NMR data for 1 are shown in SI-01.

\section{SI-19 Compound 30}
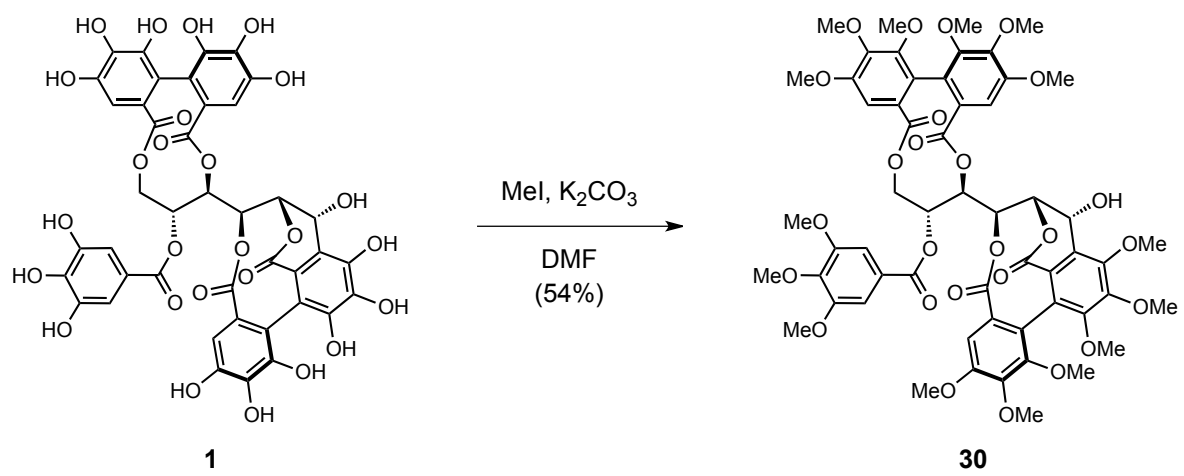

Synthesized casuarinin (1) $(6.8 \mathrm{mg}, 7.3 \mu \mathrm{mol})$, potassium carbonate $(20.0 \mathrm{mg}, 145 \mu \mathrm{mol})$, and methyl iodide $(20.5 \mathrm{mg}, 145 \mu \mathrm{mol})$ in DMF $(0.7 \mathrm{~mL})$ was stirred for $22 \mathrm{~h}$ at rt. The reaction was quenched with $1 \mathrm{M}$ hydrochloric acid. The mixture was extracted with ethyl acetate. The organic layer was successively washed with 1 $\mathrm{M}$ hydrochloric acid, sat. sodium hydrogen carbonate aq, and brine. After the general drying procedure, the resulting residue was purified by $\mathrm{CC}$ (silica gel $2.5 \mathrm{~g}$, hexane/ethyl acetate $=1 / 1$ to $1 / 2)$ to afford $\mathbf{3 0}(4.5 \mathrm{mg}, 3.9$ $\mu \mathrm{mol}, 54 \%$ yield $)$ as a colorless amorphous solid; $[\alpha]_{\mathrm{D}}{ }^{25}-32\left(c 0.20, \mathrm{CHCl}_{3}\right) ;{ }^{13} \mathrm{C} \mathrm{NMR}\left(126 \mathrm{MHz}, \mathrm{CDCl}_{3}\right.$, $\left.21.8^{\circ} \mathrm{C}\right) \delta 168.3(\mathrm{~s}, 1 \mathrm{C}, \mathrm{HHDP}), 168.0$ (s, 1C, HHDP), 167.8 (s, 1C, HHDP), 165.1 (s, 1C, G), 162.6 (s, 1C, HHDP), 153.7 (s, 1C, HHDP), 153.4 (s, 1C, HHDP), 153.3 (s, 1C, HHDP), 153.1 (s, 1C, HHDP), 153.0 (s, 2C, G), 152.4 (s, 1C, HHDP), 152.2 (s, 1C, HHDP), 151.4 (s, 1C, HHDP), 149.9 (s, 2C, HHDP), 144.9 (s, 1C, HHDP), 143.9 (s, 1C, HHDP), 143.0 (s, 1C, HHDP), 142.7 (s, 1C, G), 129.4 (s, 1C, HHDP), 128.7 (s, 1C, HHDP), 127.2 (s, 1C, HHDP), 126.6 (s, 1C, HHDP), 126.4 (s, 1C, HHDP), 124.1 (s, 1C, G), 123.5 (s, 1C, HHDP), 122.4 (s, 1C, HHDP), 121.7 (s, 1C, HHDP), 120.8 (s, 1C, HHDP), 107.11 (d, 2C, G), 107.07 (d, 1C, HHDP), 105.0 (d, 1C, HHDP), 103.6 (d, 1C, HHDP), 75.5 (d, 1C, C-2), 72.9 (d, 1C, C-4), 70.6 (d, 1C, C-5), 69.1 (d, 1C, C-3), 66.3 (d, 1C, C-1), 64.2 (t, 1C, C-6), 62.1 (q, 1C, Me), 61.15 (q, 2C, Me), 61.06 (q, 2C, Me), 60.98 (q, $1 \mathrm{C}, \mathrm{Me}$ ), 60.94 (q, 1C, Me), 60.90 (q, 1C, Me), 60.86 (q, 1C, Me), 60.1 (q, 1C, Me), 56.5 (q, 1C, Me), 56.4 (q, 2C, $\mathrm{Me}$ ), 56.2 (q, 1C, Me), 55.9 (q, 1C, Me); HRMS (ESI) $m / z[\mathrm{M}+\mathrm{Na}]^{+}$calcd for $\mathrm{C}_{56} \mathrm{H}_{56} \mathrm{O}_{26} \mathrm{Na}$ 1169.3114, found 1169.3130; ${ }^{1} \mathrm{H}$ NMR data for $\mathbf{3 0}$ are shown in SI-02. 


\section{Spectra}

\section{SI-20 Compound 21}

${ }^{1} \mathrm{H} \mathrm{NMR}\left(500 \mathrm{MHz}, \mathrm{CDCl}_{3}\right.$ )

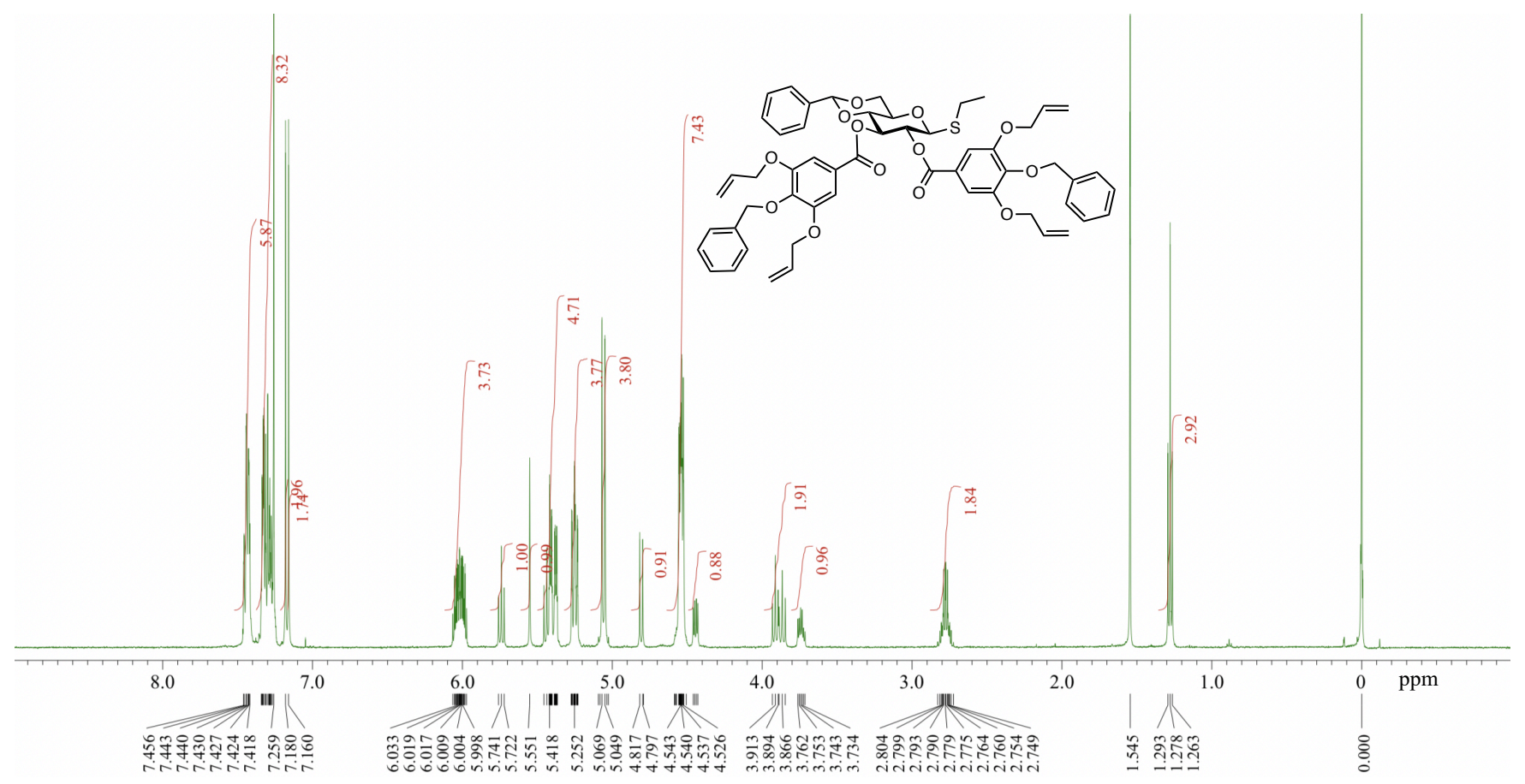

${ }^{13} \mathrm{C}$ NMR $\left(126 \mathrm{MHz}, \mathrm{CDCl}_{3}\right)$

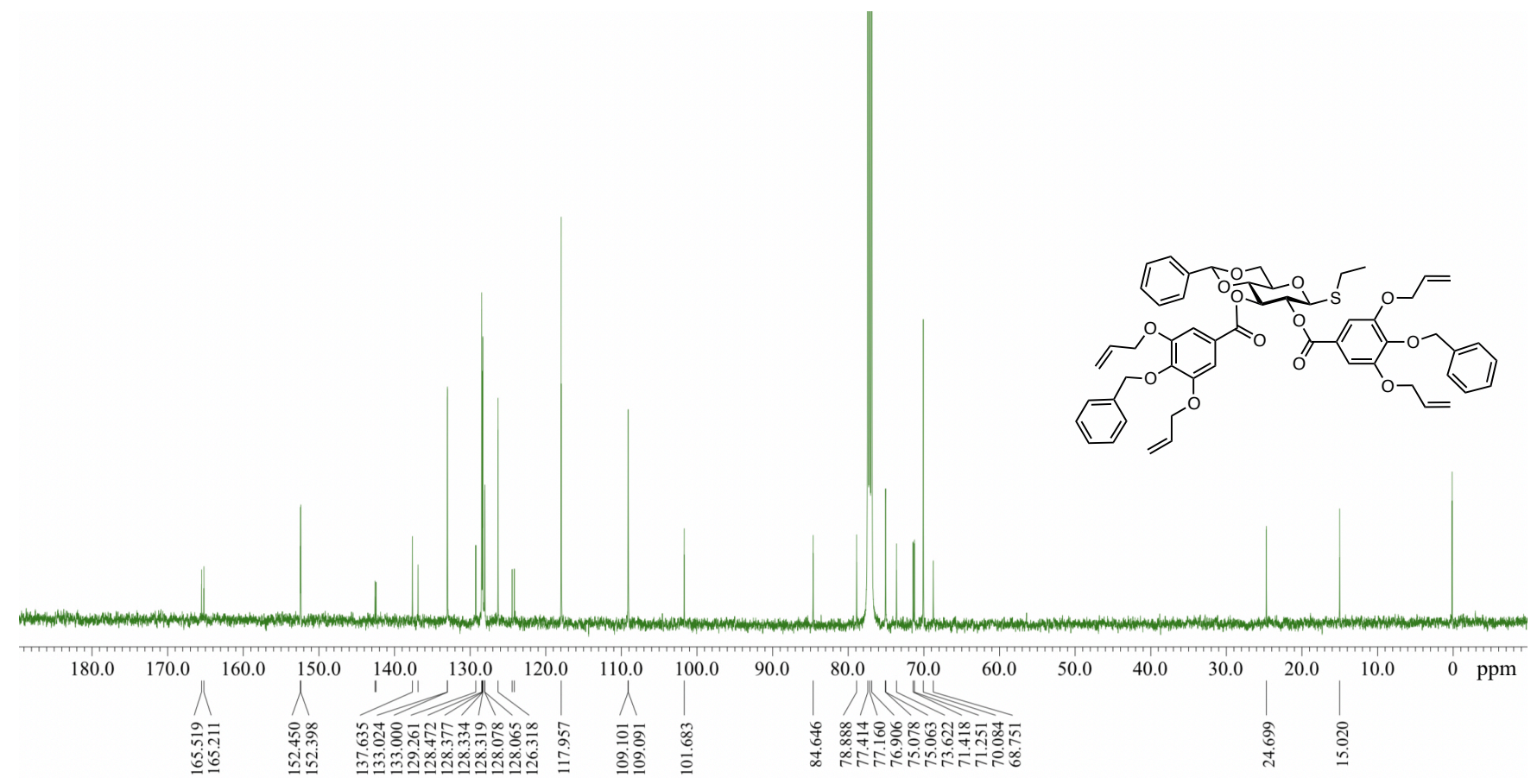




\section{SI-21 Compound 22}

${ }^{1} \mathrm{H} \mathrm{NMR}\left(500 \mathrm{MHz}, \mathrm{CDCl}_{3}\right)$

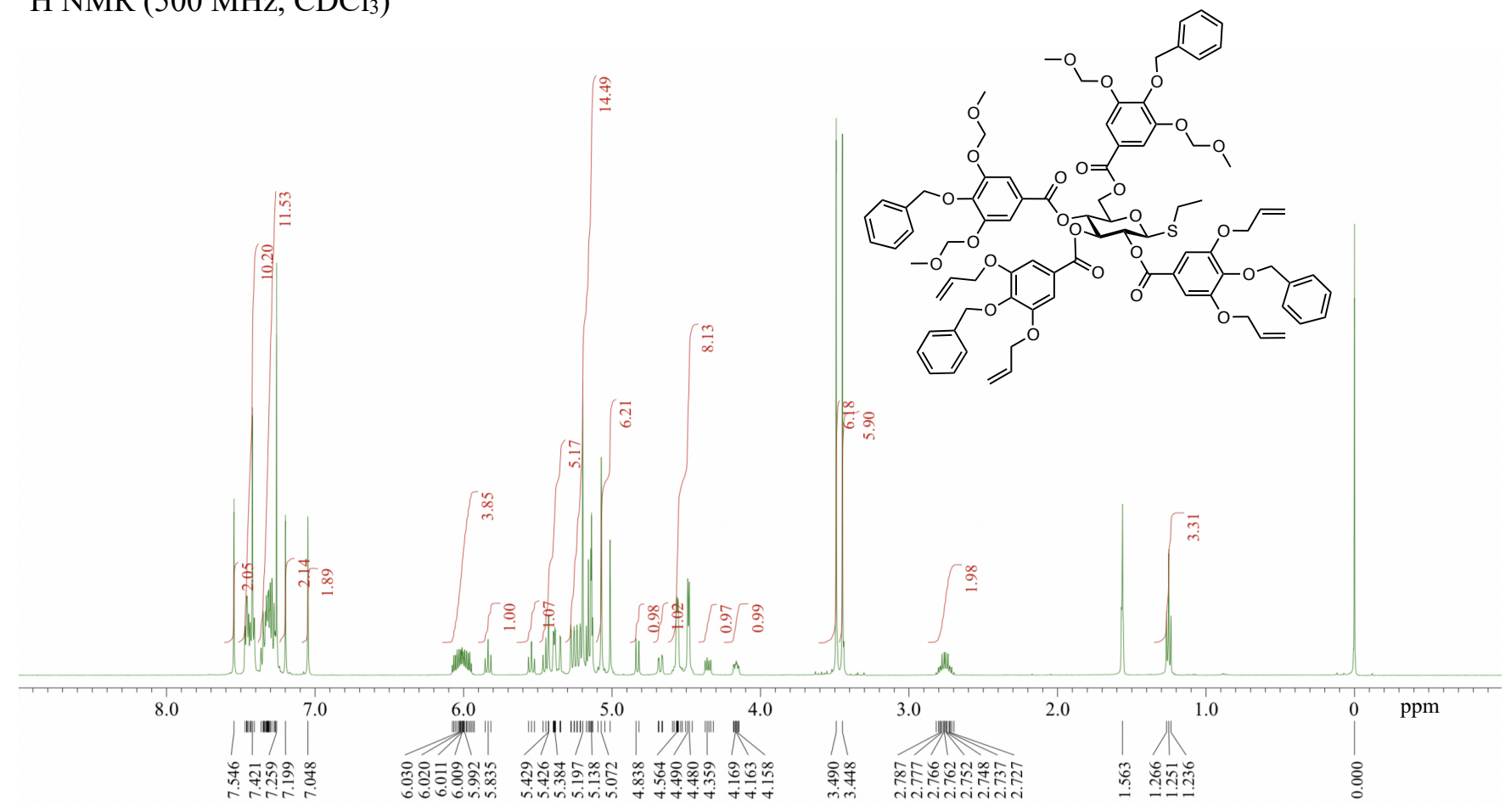

${ }^{13} \mathrm{C}$ NMR $\left(126 \mathrm{MHz}, \mathrm{CDCl}_{3}\right)$

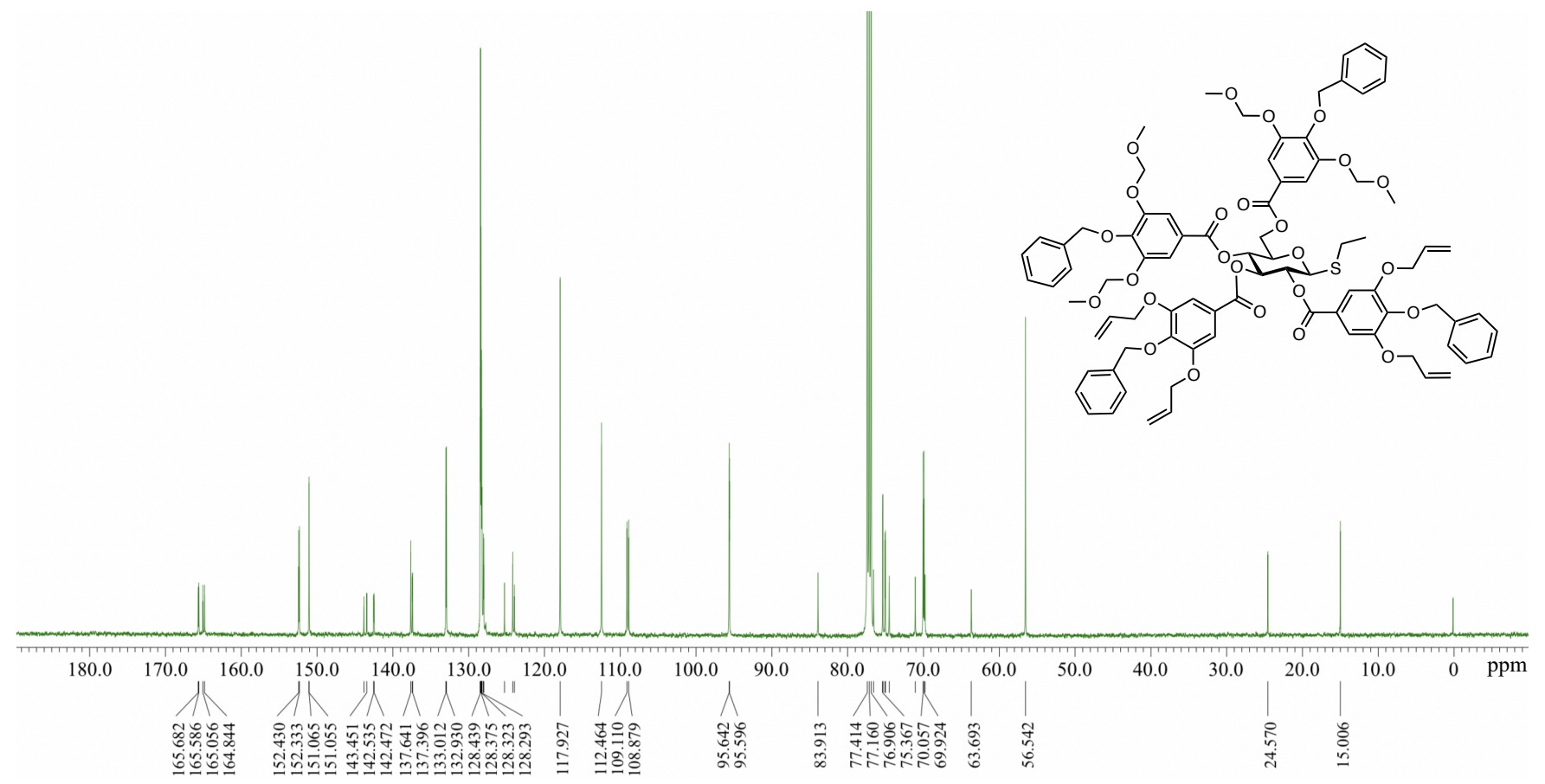




\section{SI-22 Compound 23}

${ }^{1} \mathrm{H}$ NMR (500 MHz, $\mathrm{CDCl}_{3}$ )

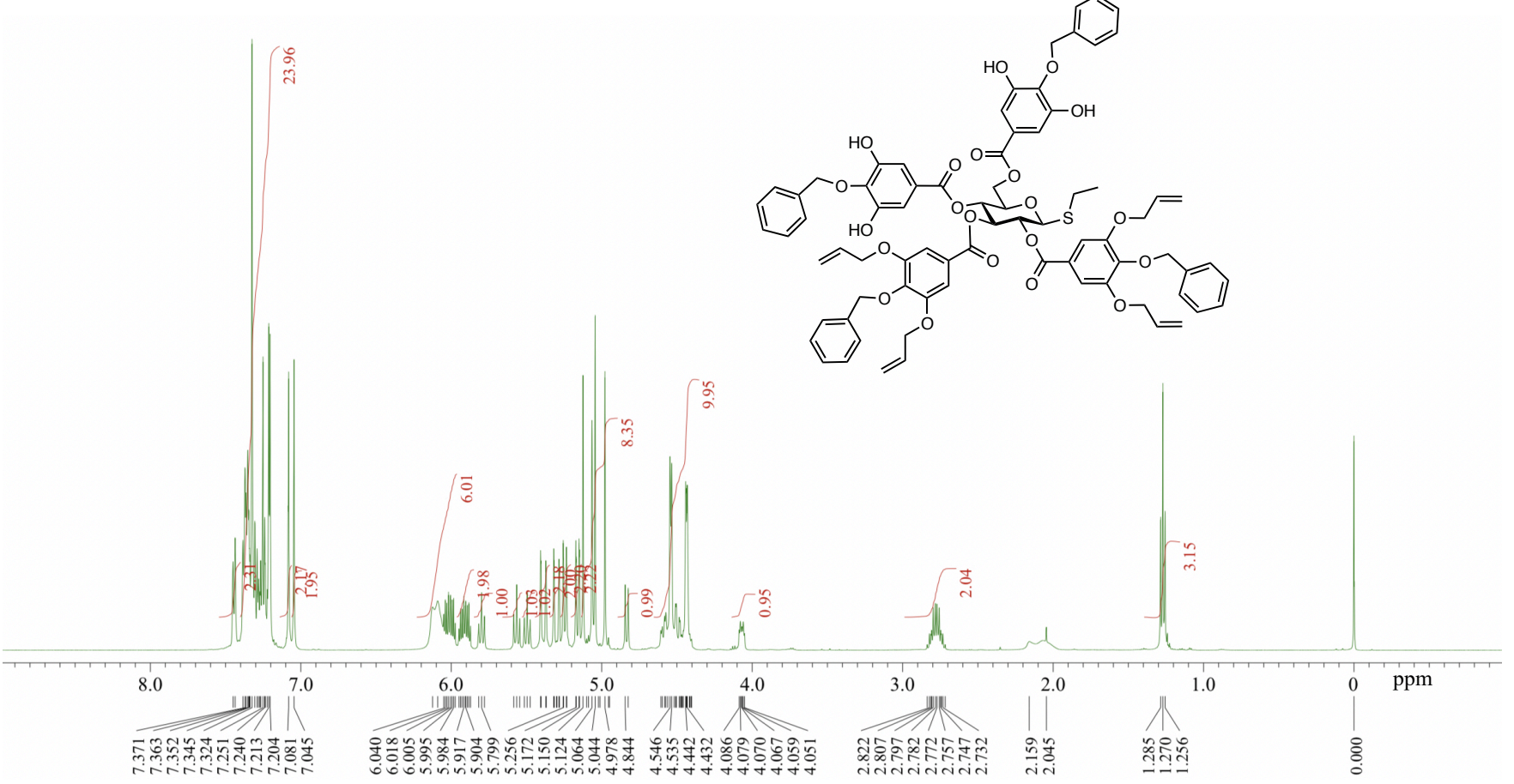

${ }^{13} \mathrm{C}$ NMR $\left(126 \mathrm{MHz}, \mathrm{CDCl}_{3}\right)$

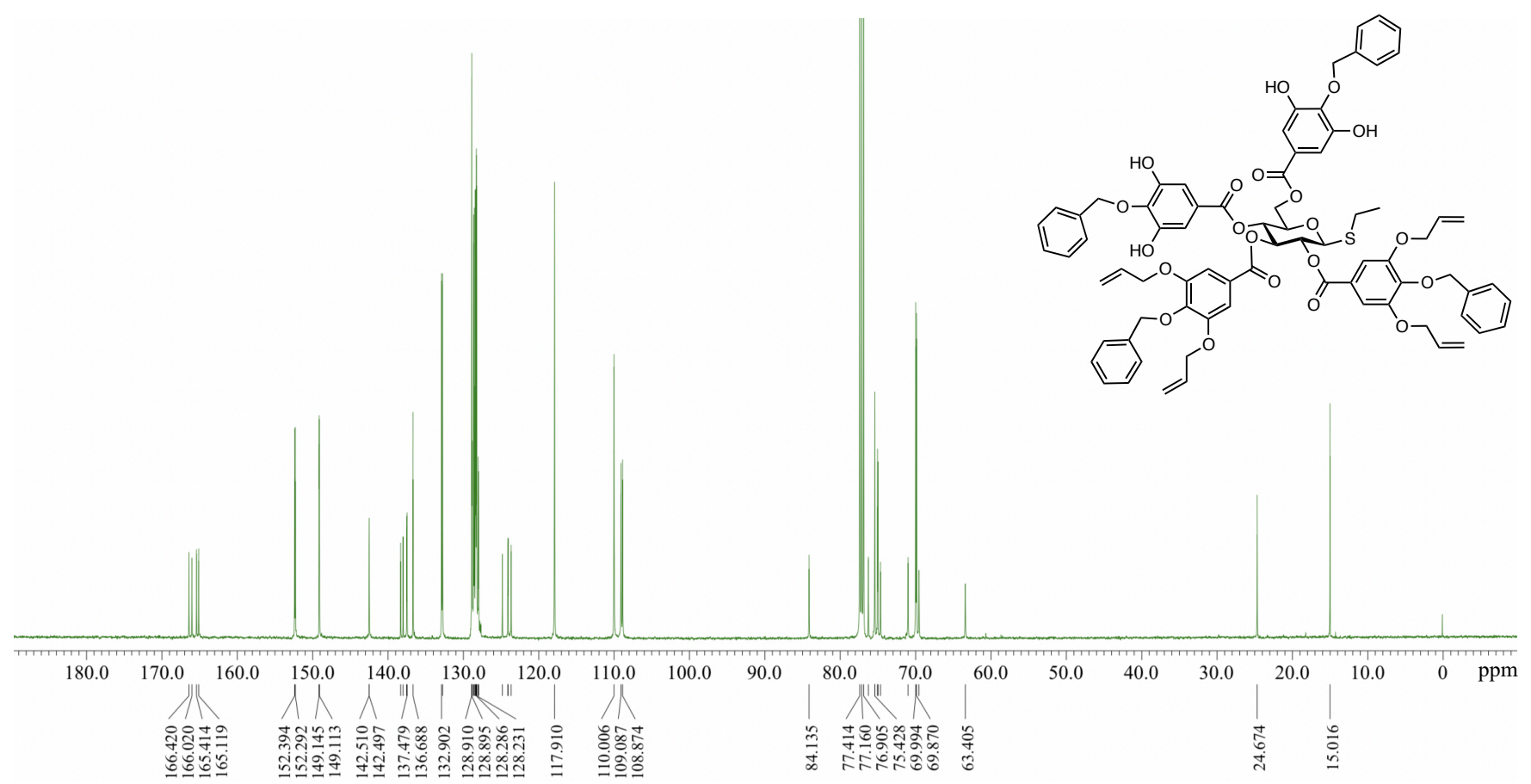




\section{SI-23 Compound 23 (recovered)}

${ }^{1} \mathrm{H}$ NMR (500 MHz, $\mathrm{CDCl}_{3}$ )

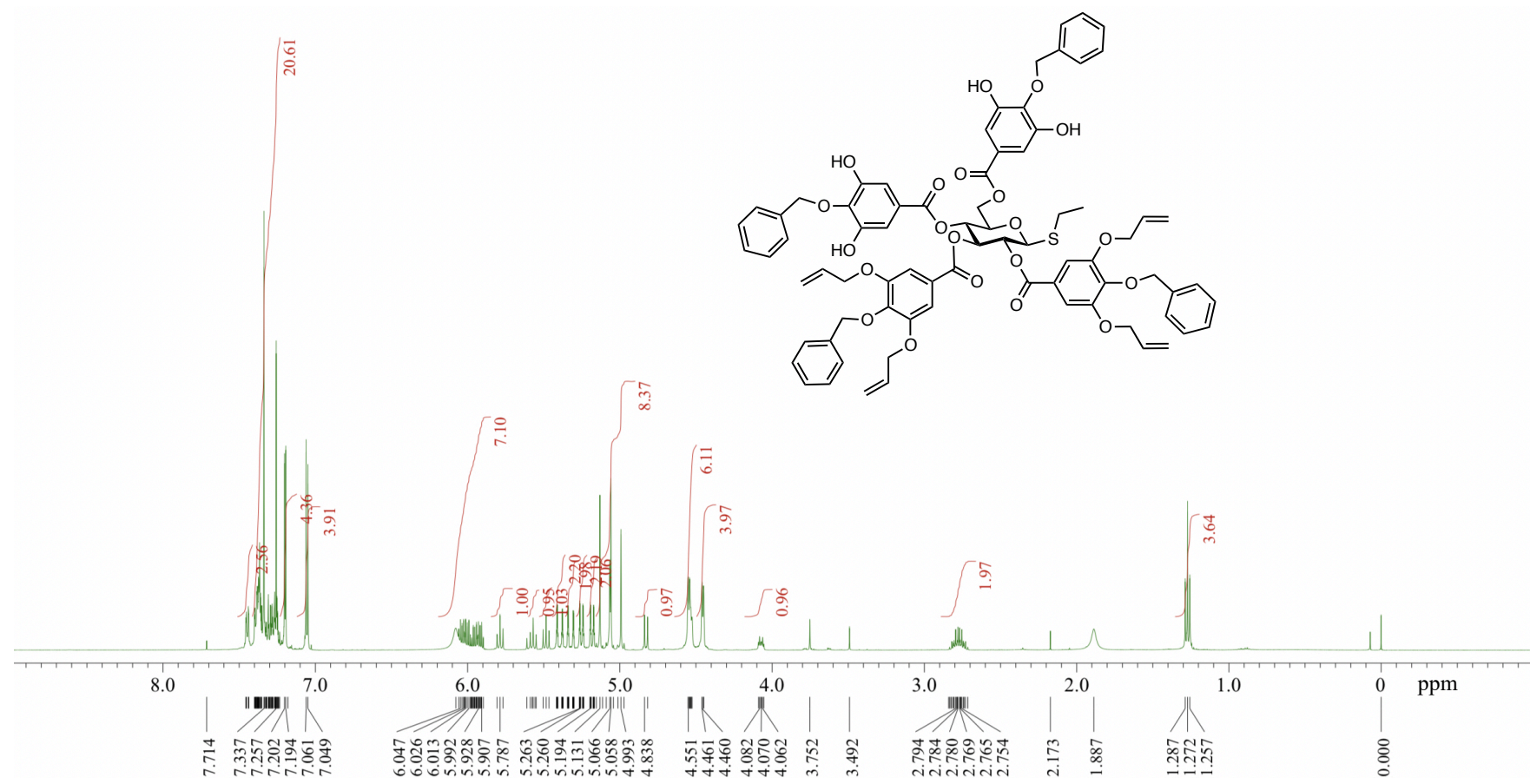

${ }^{13} \mathrm{C}$ NMR (126 MHz, $\left.\mathrm{CDCl}_{3}\right)$

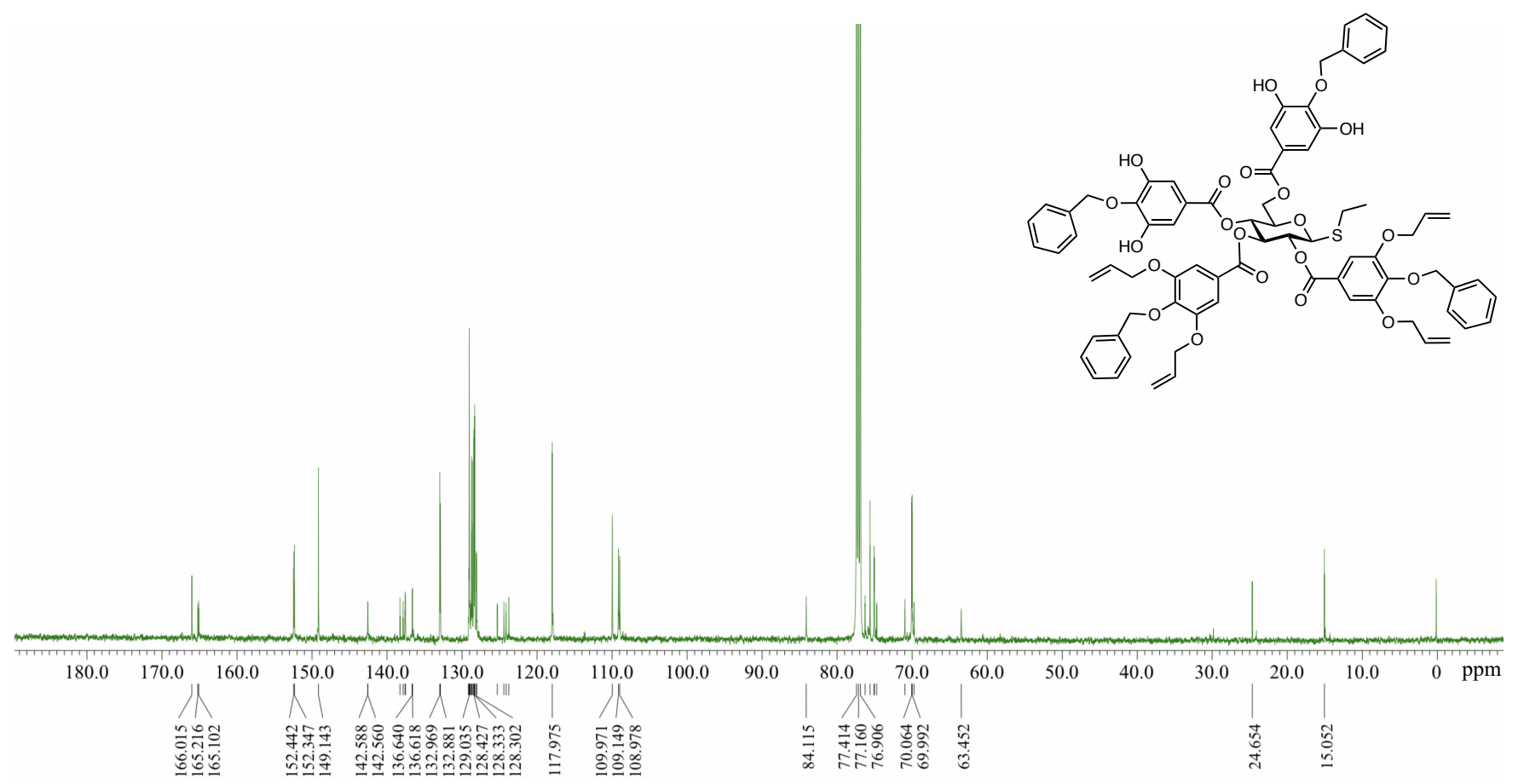




\section{SI-24 Compound 24}

${ }^{1} \mathrm{H}$ NMR (500 MHz, $\mathrm{CDCl}_{3}$ )

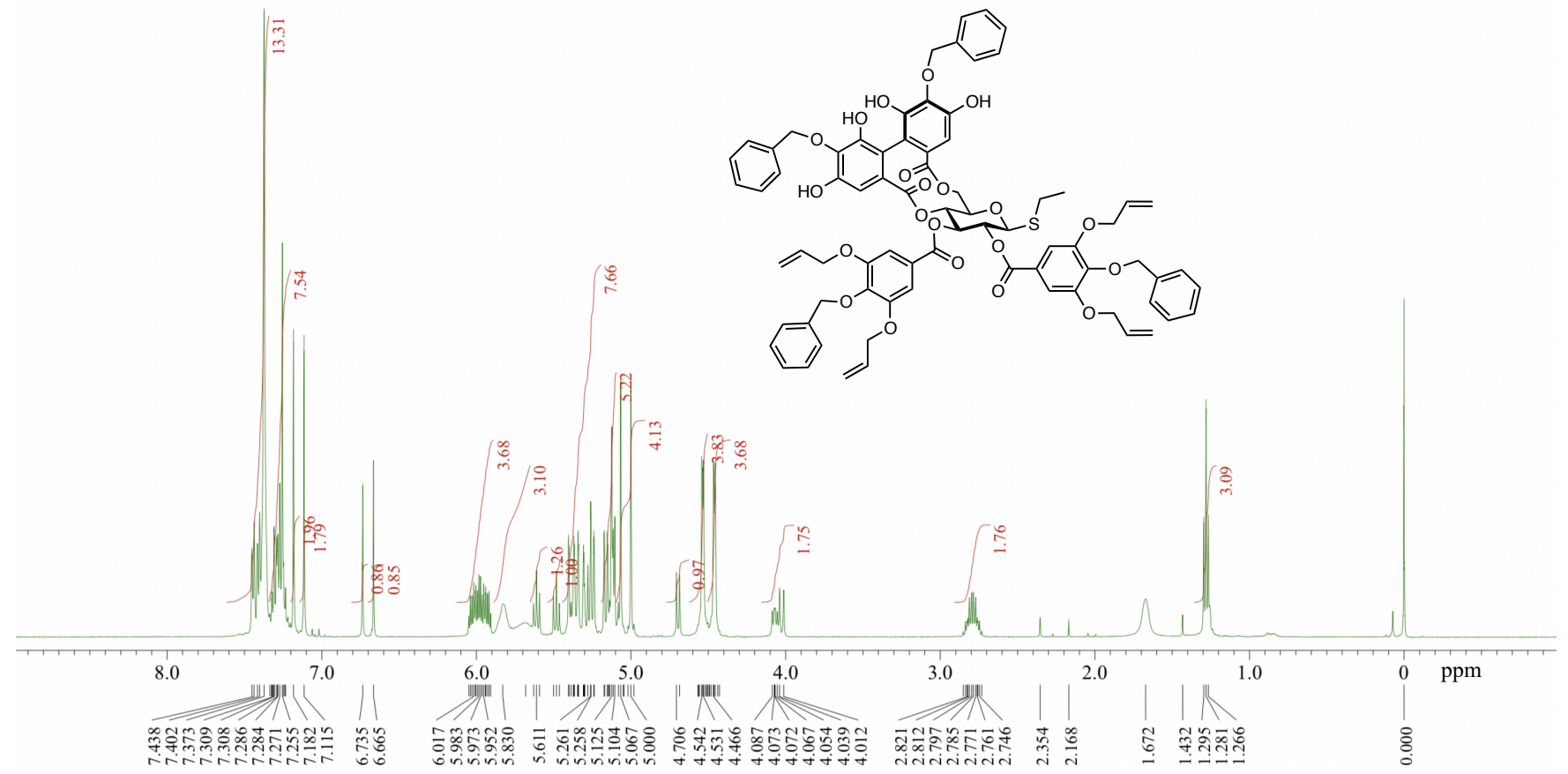

${ }^{13} \mathrm{C}$ NMR (126 MHz, $\left.\mathrm{CDCl}_{3}\right)$

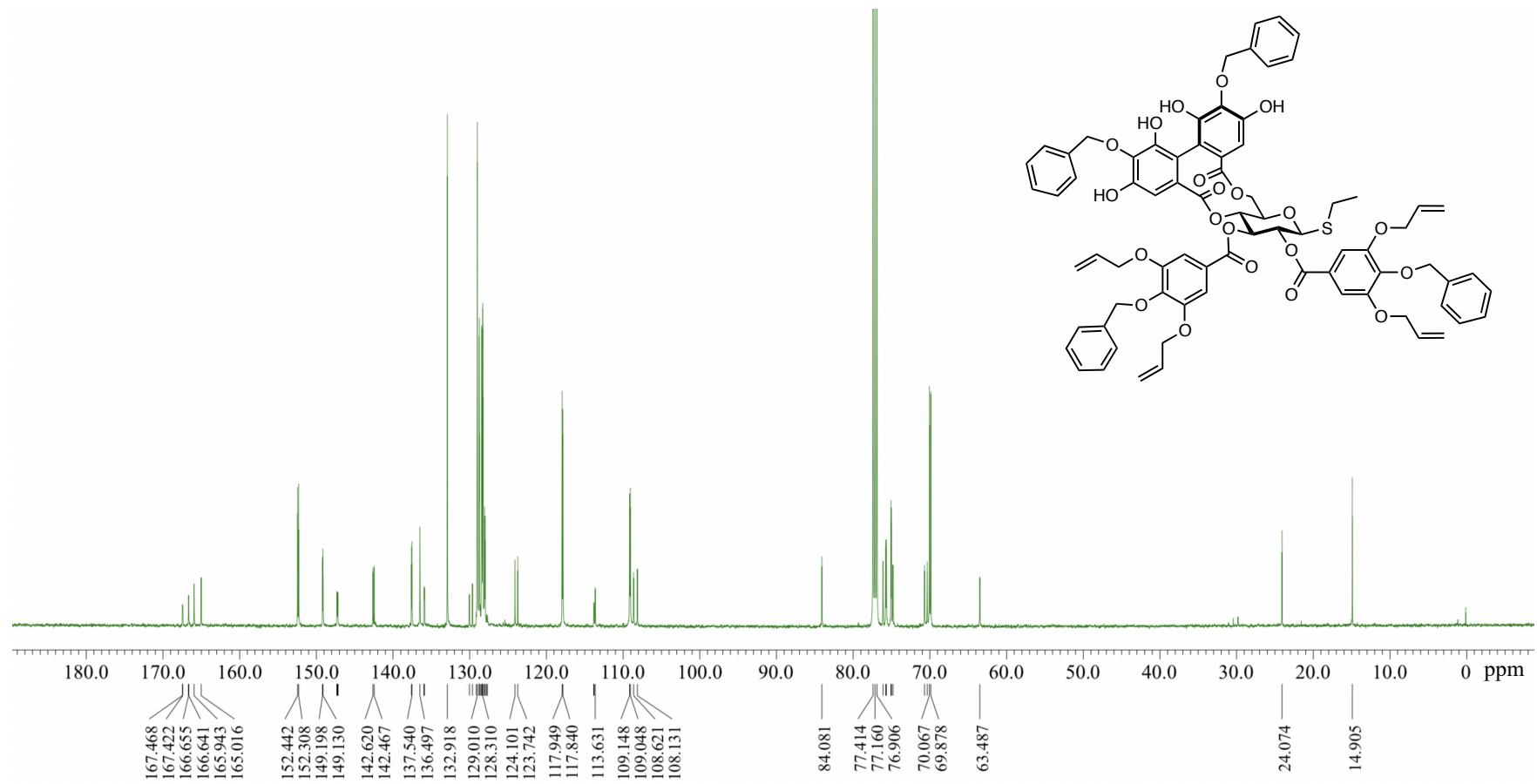




\section{SI-25 Compound 31}

${ }^{1} \mathrm{H}$ NMR (500 MHz, $\mathrm{CDCl}_{3}$ )

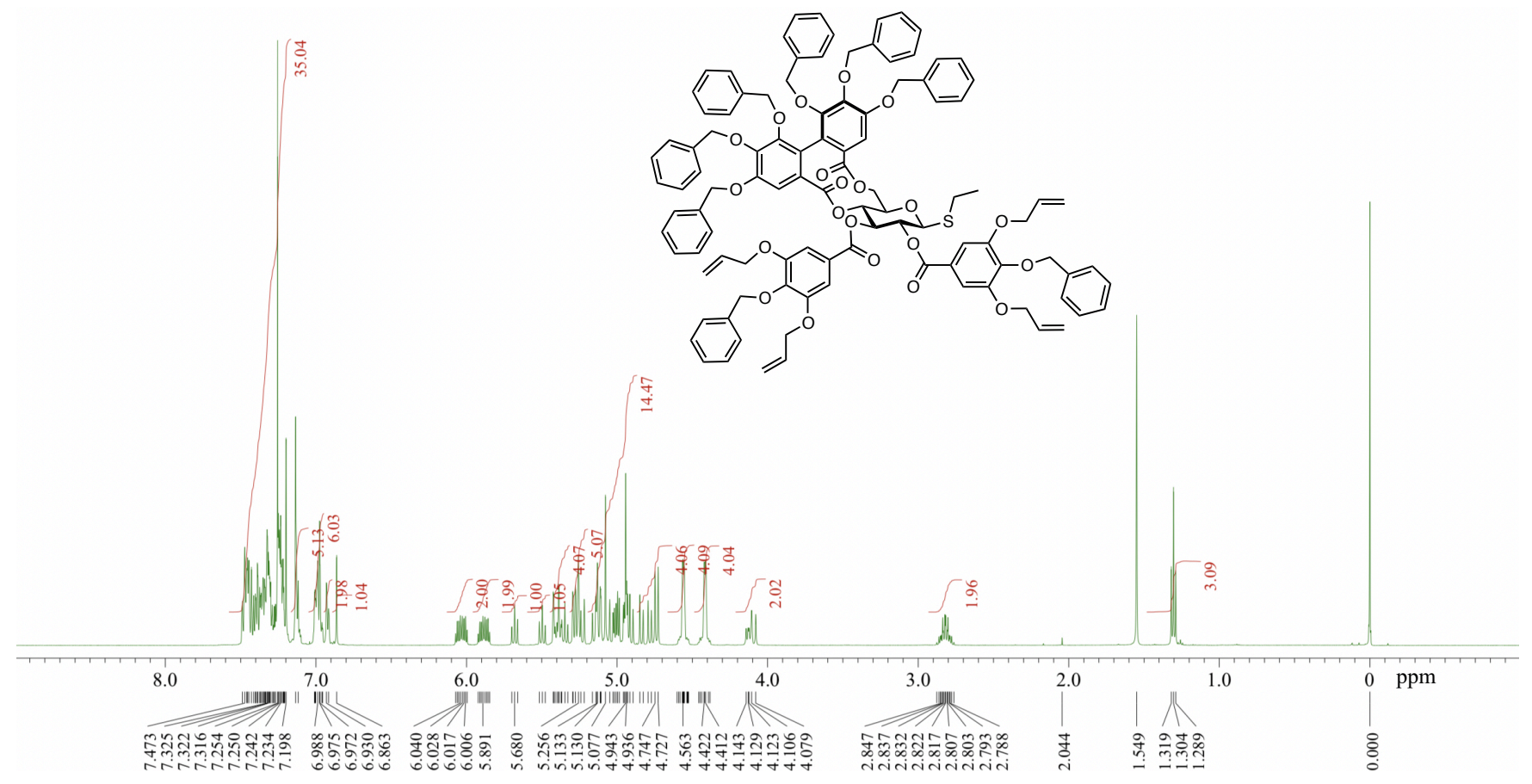

${ }^{13} \mathrm{C}$ NMR $\left(126 \mathrm{MHz}, \mathrm{CDCl}_{3}\right)$

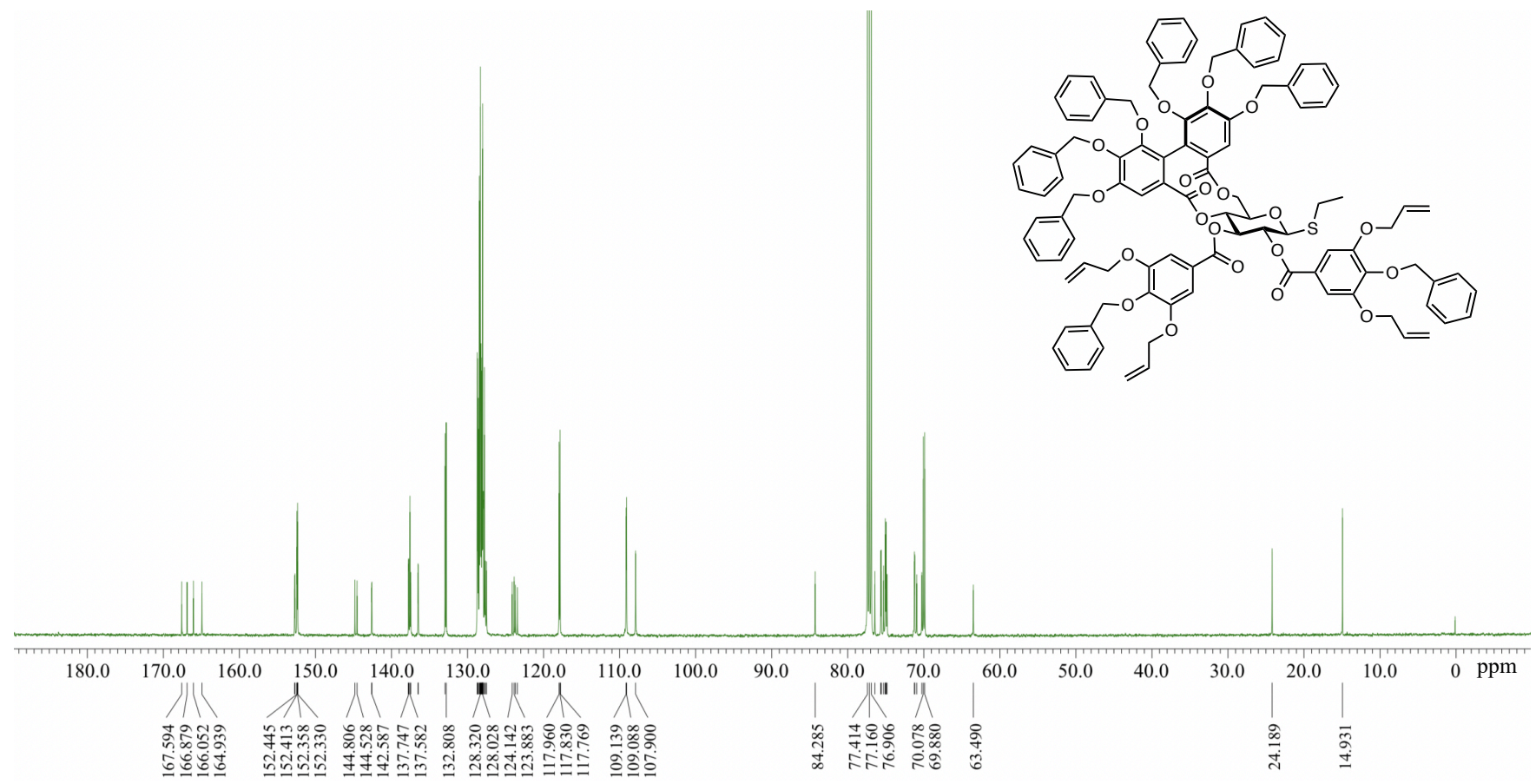




\section{SI-26 Compound 25}

${ }^{1} \mathrm{H}$ NMR (500 MHz, $\mathrm{CDCl}_{3}$ )

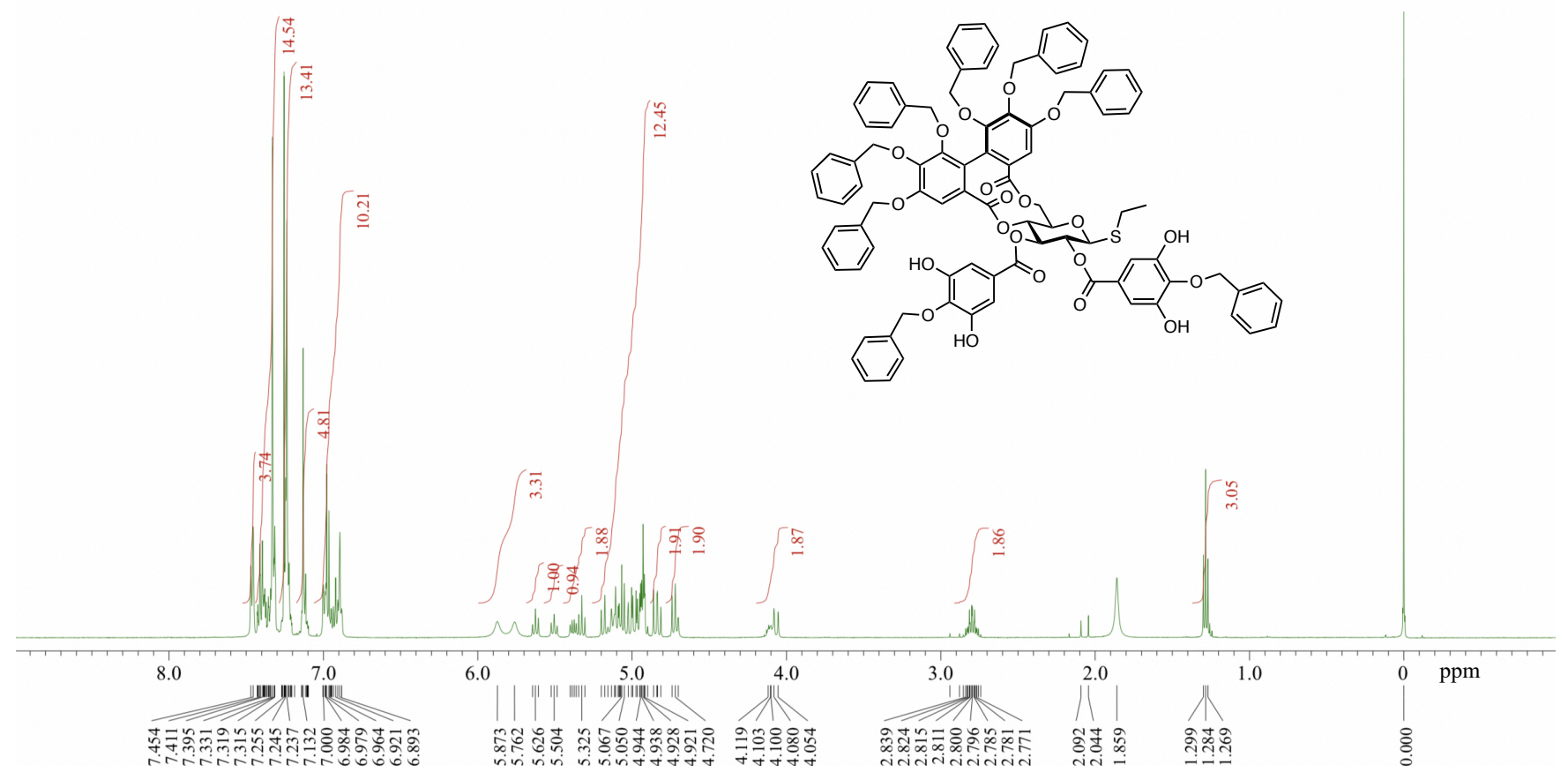

${ }^{13} \mathrm{C}$ NMR $\left(126 \mathrm{MHz}, \mathrm{CDCl}_{3}\right)$

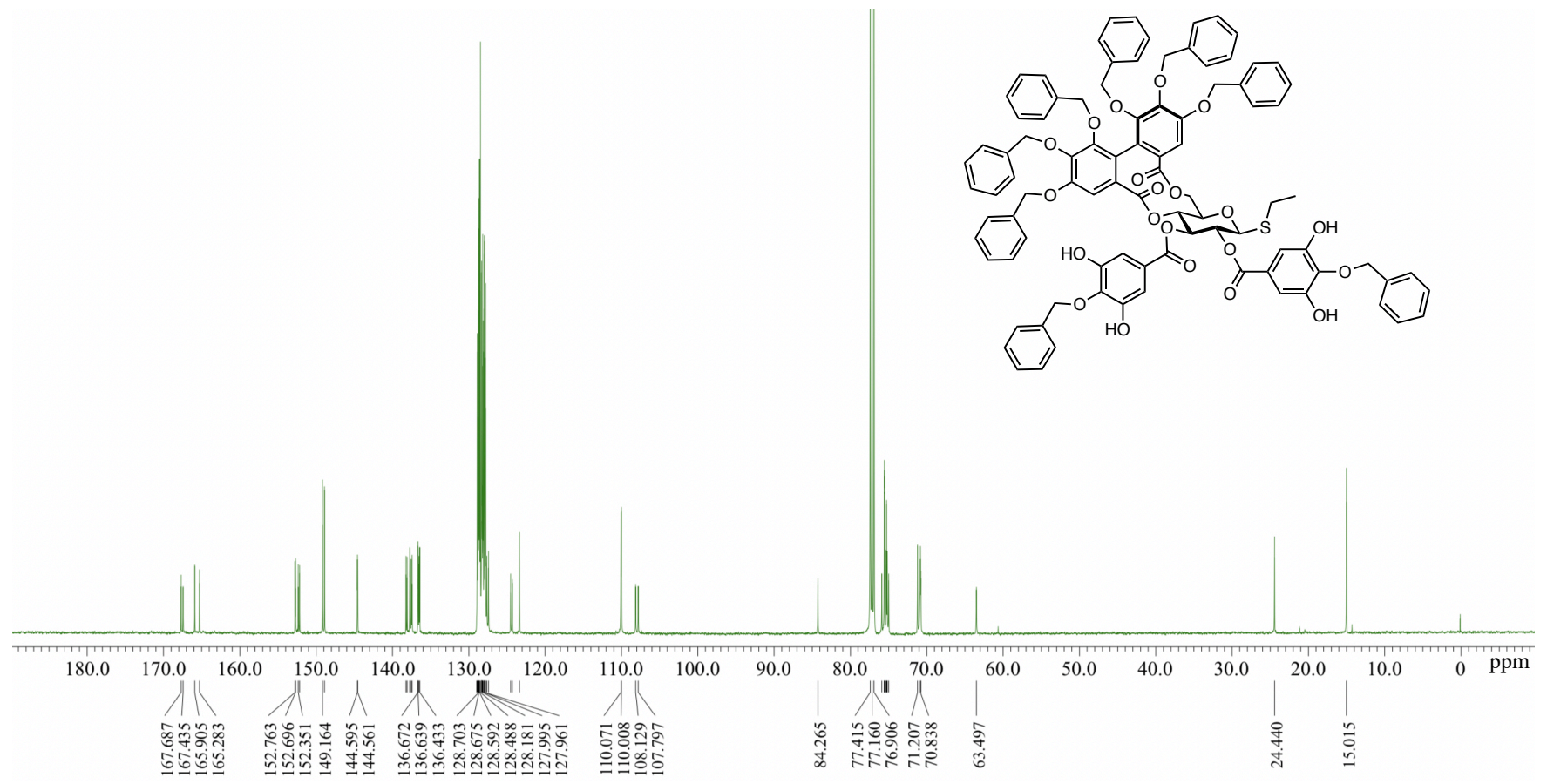




\section{SI-27 Compound 26}

${ }^{1} \mathrm{H}$ NMR (500 MHz, $\mathrm{CDCl}_{3}$ )

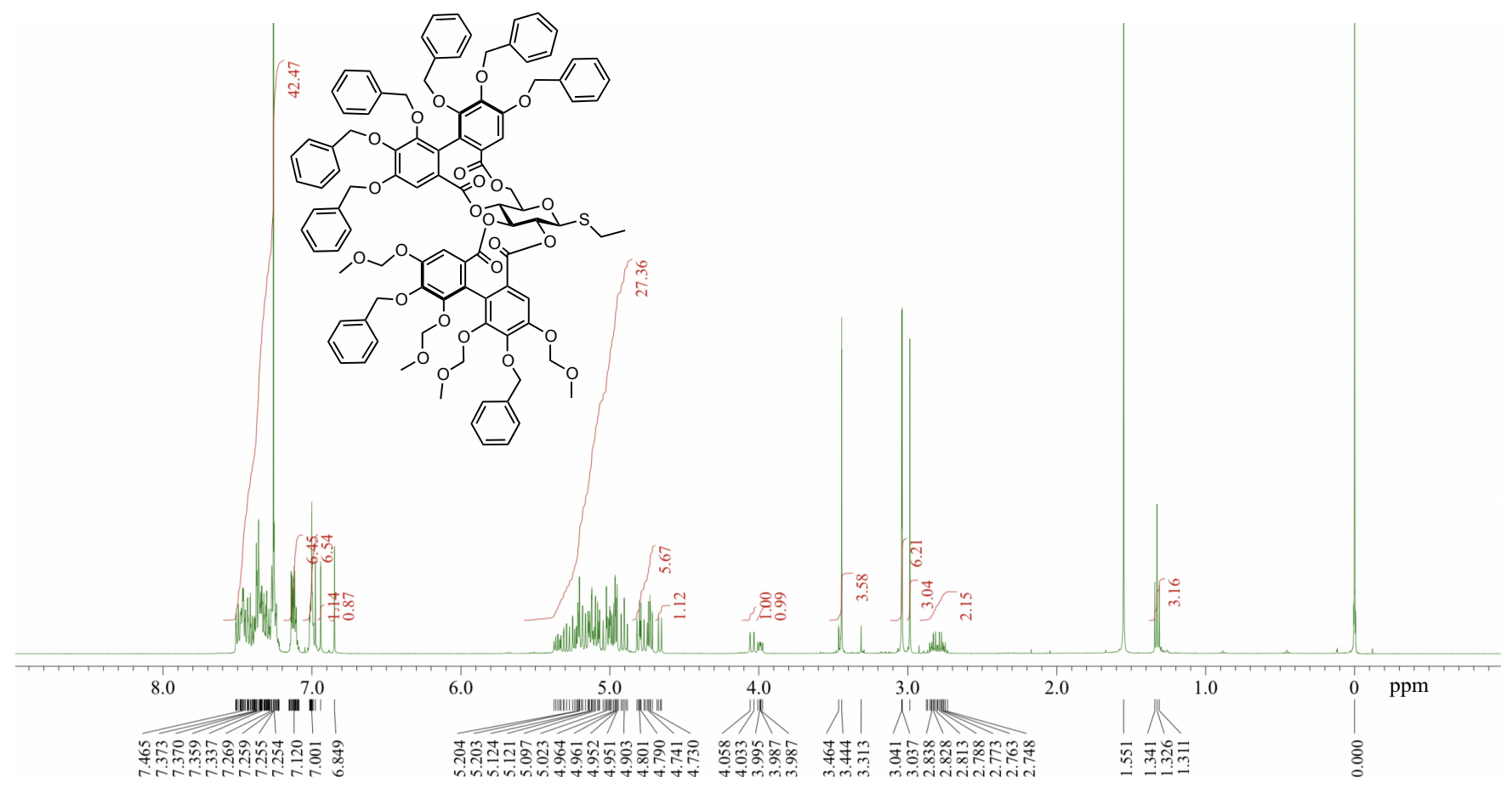

${ }^{13} \mathrm{C}$ NMR (126 MHz, $\left.\mathrm{CDCl}_{3}\right)$

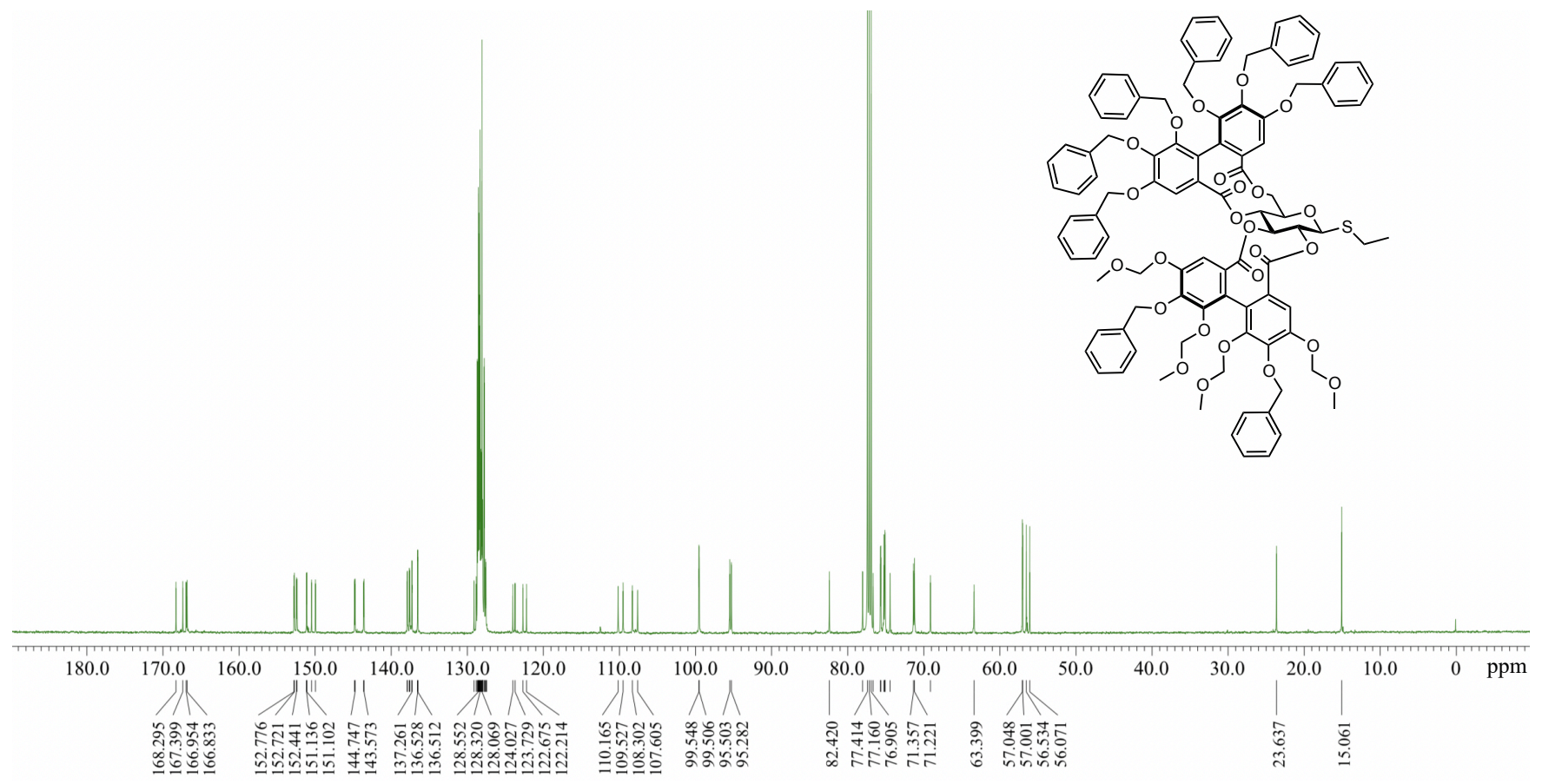




\section{SI-28 Compound 27}

${ }^{1} \mathrm{H}$ NMR (500 MHz, $\mathrm{CDCl}_{3}$ )

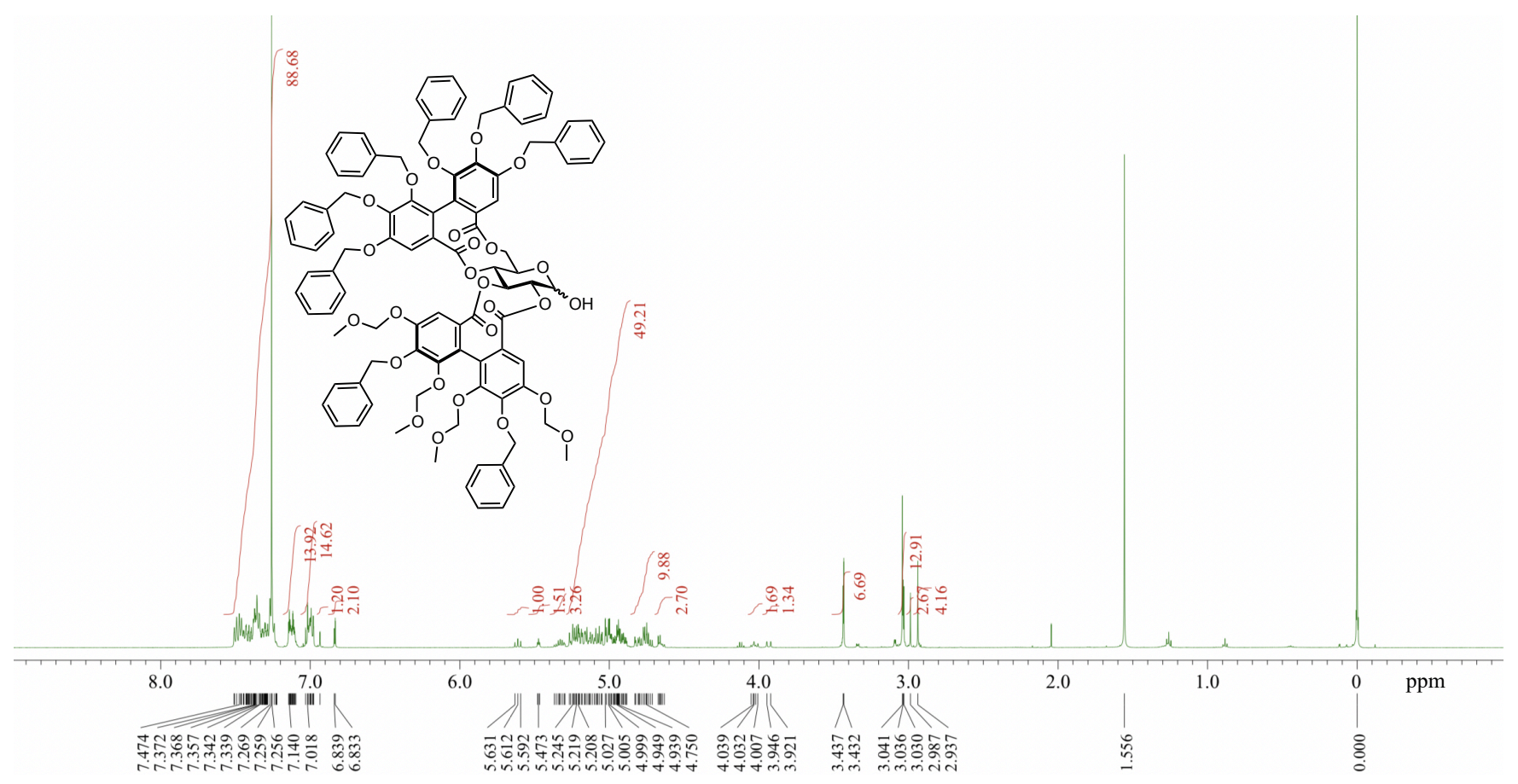

\section{SI-29 Compound 28}

${ }^{1} \mathrm{H}$ NMR (500 MHz, $\mathrm{CDCl}_{3}$ )

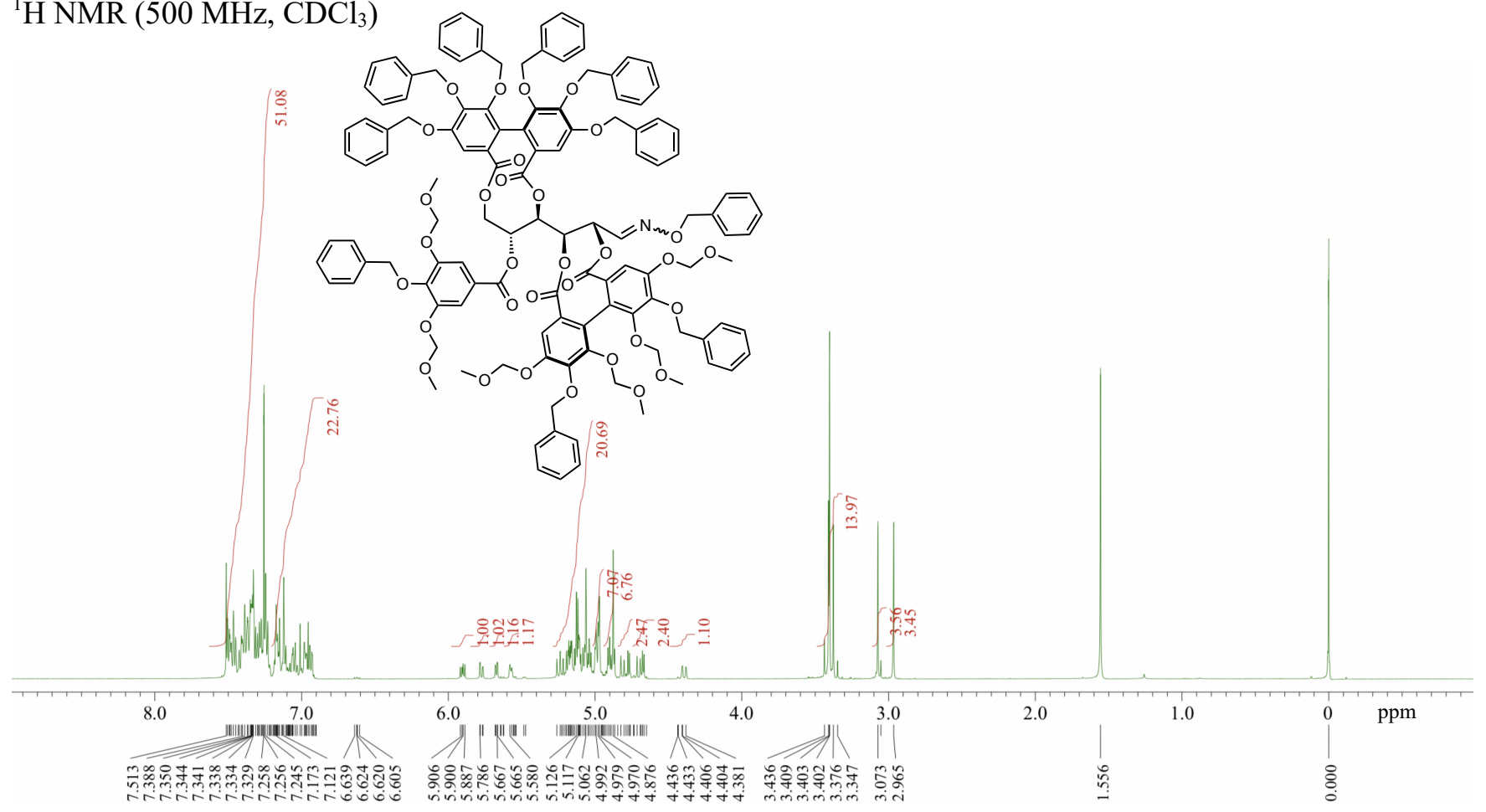




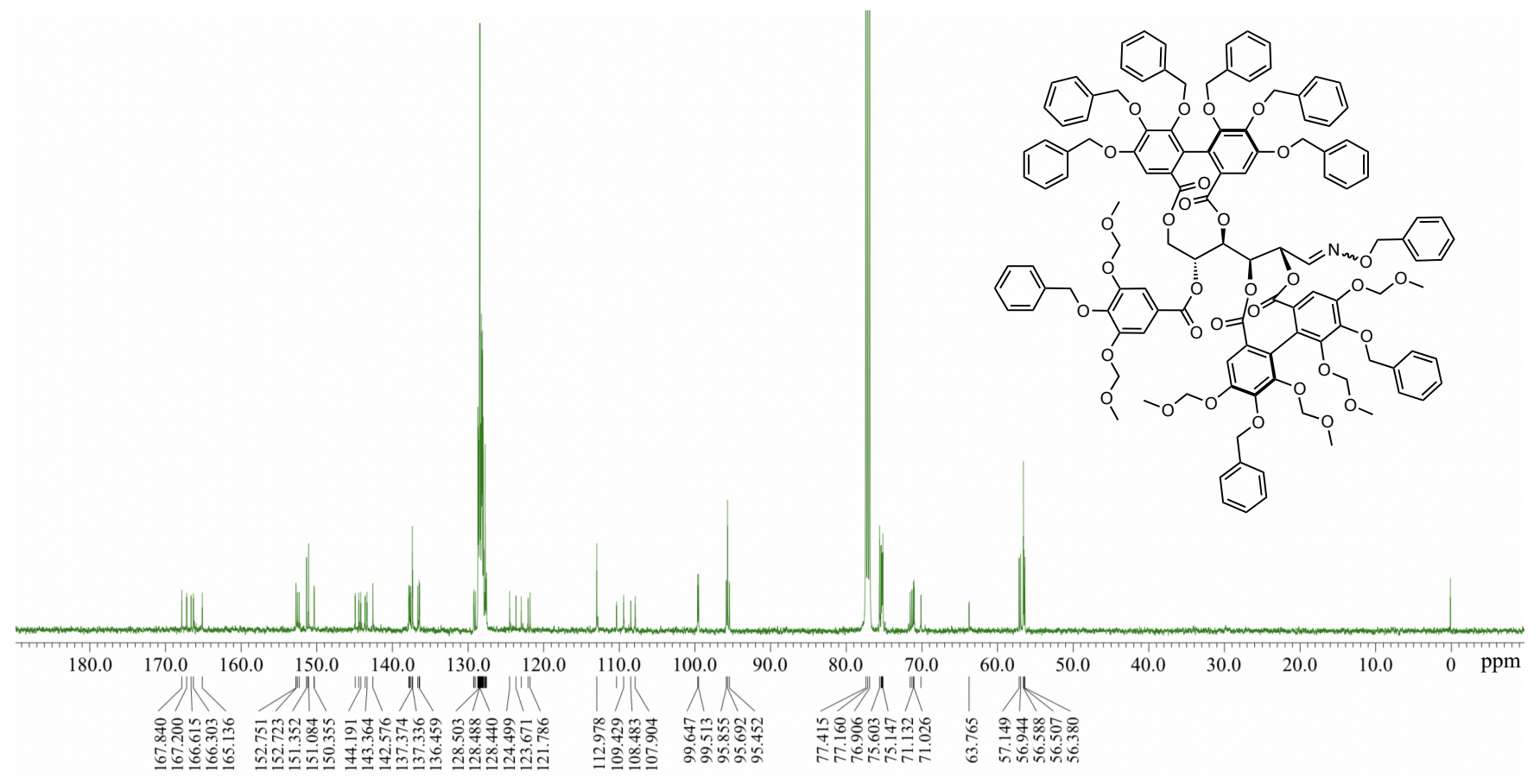

\section{SI-30 Compound 29}

${ }^{1} \mathrm{H}$ NMR $\left(500 \mathrm{MHz}, \mathrm{CDCl}_{3}\right)$

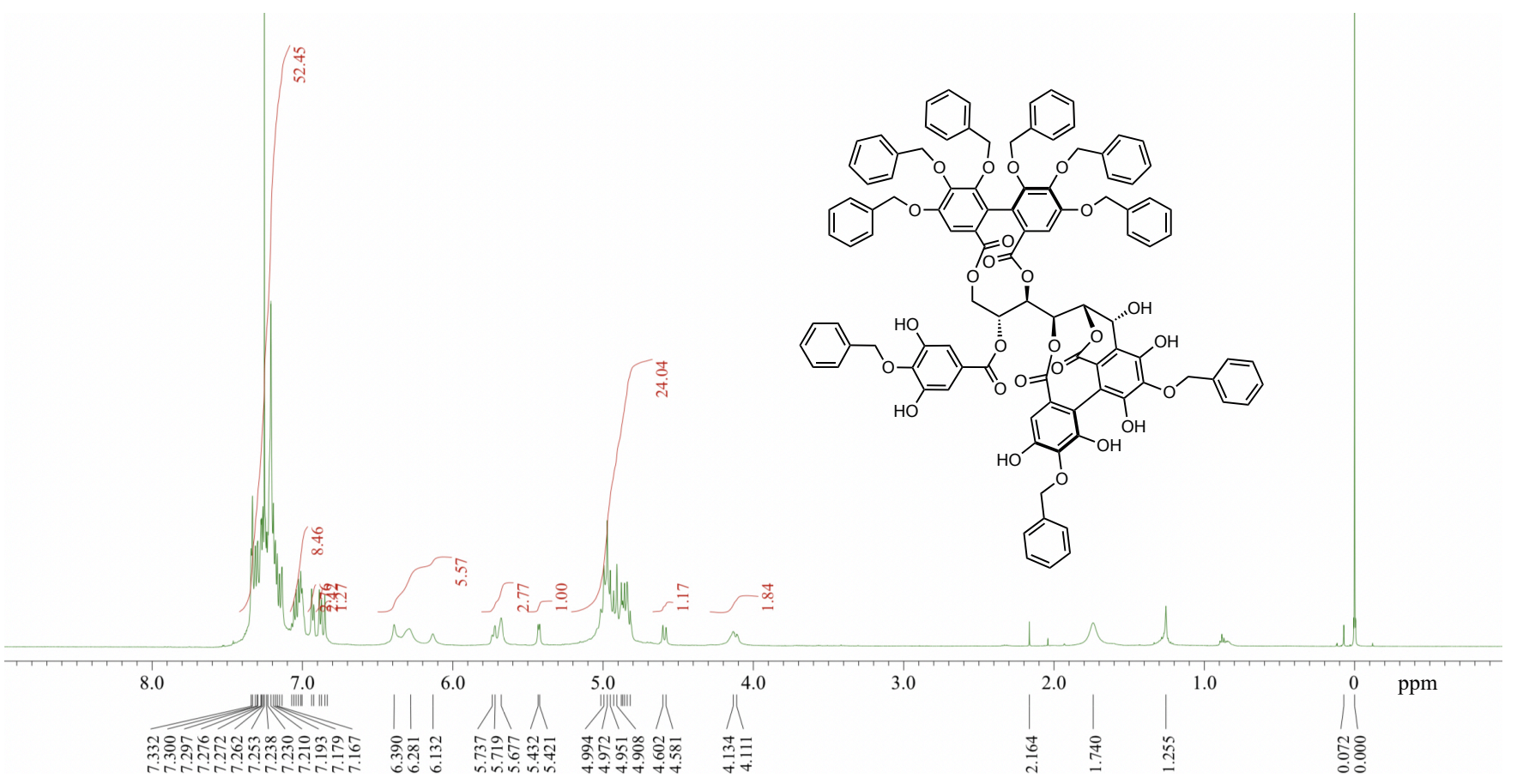


${ }^{13} \mathrm{C}$ NMR (126 MHz, $\left.\mathrm{CDCl}_{3}\right)$

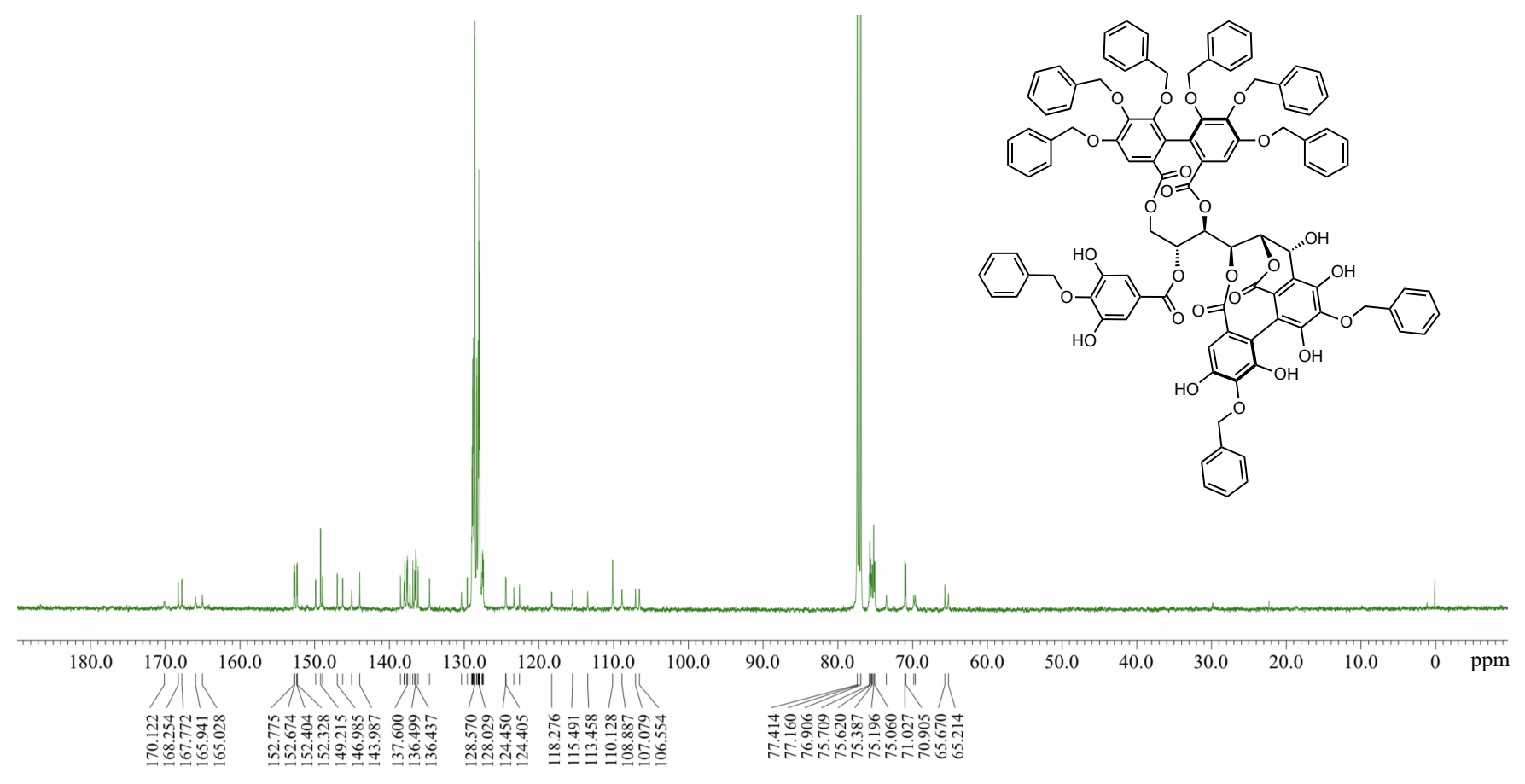

\section{SI-31 Casuarinin (1)}

${ }^{1} \mathrm{H}$ NMR (500 MHz, acetone- $d_{6}$ )

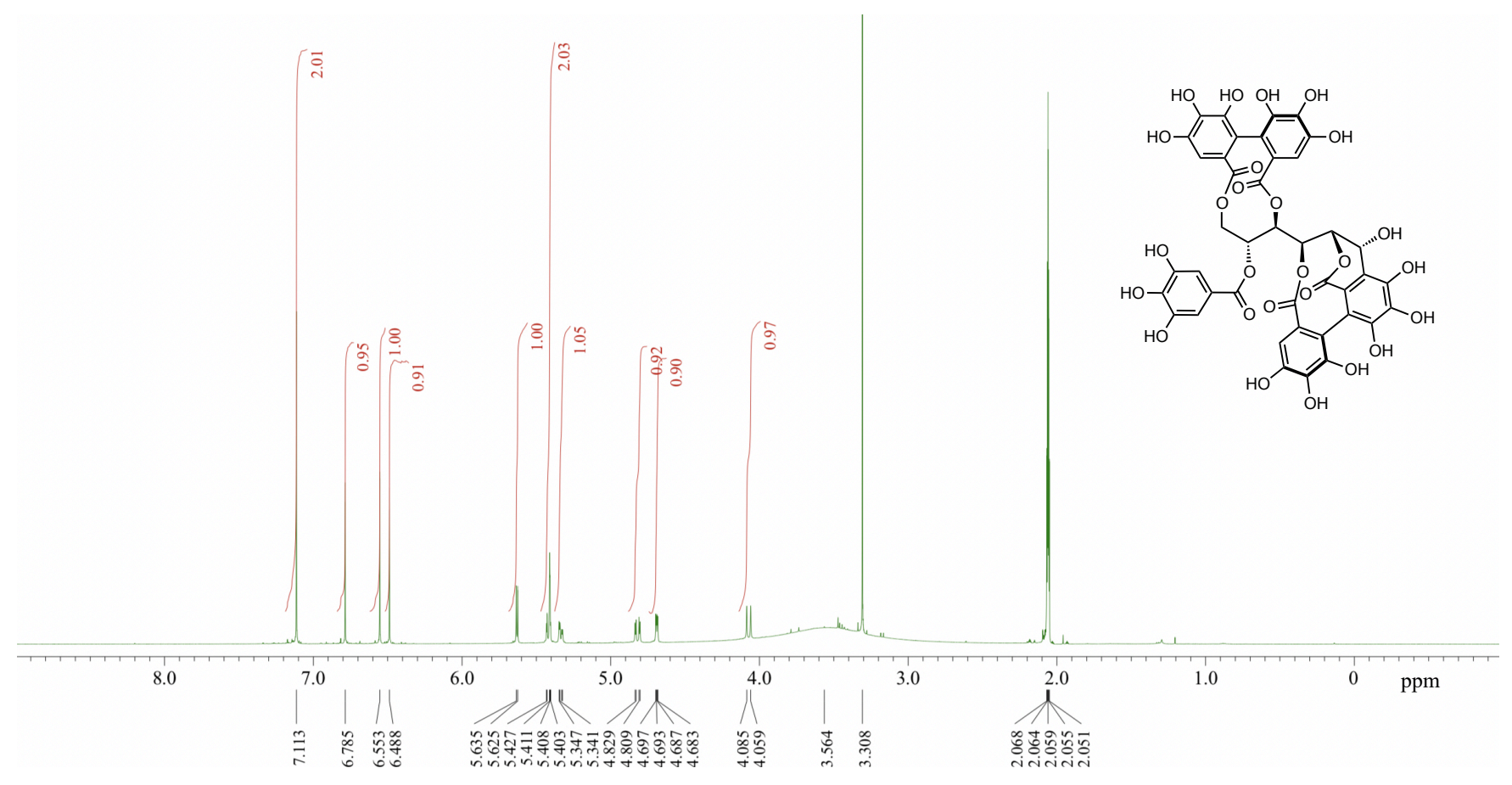


${ }^{13} \mathrm{C}$ NMR (126 MHz, acetone- $d_{6}$ )

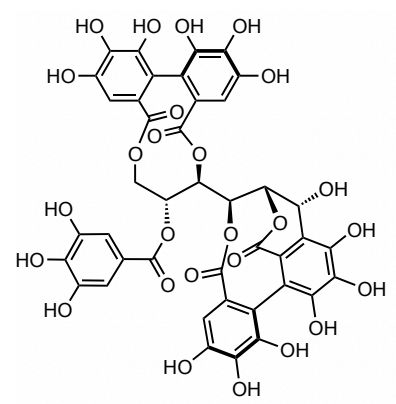

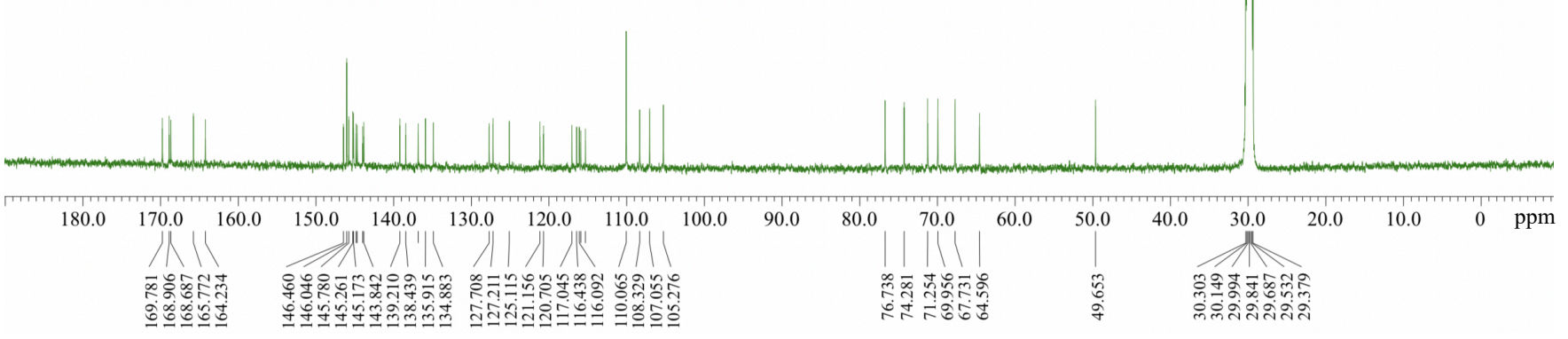

\section{SI-32 Compound 30}

${ }^{1} \mathrm{H} \mathrm{NMR}\left(500 \mathrm{MHz}, \mathrm{CDCl}_{3}\right)$

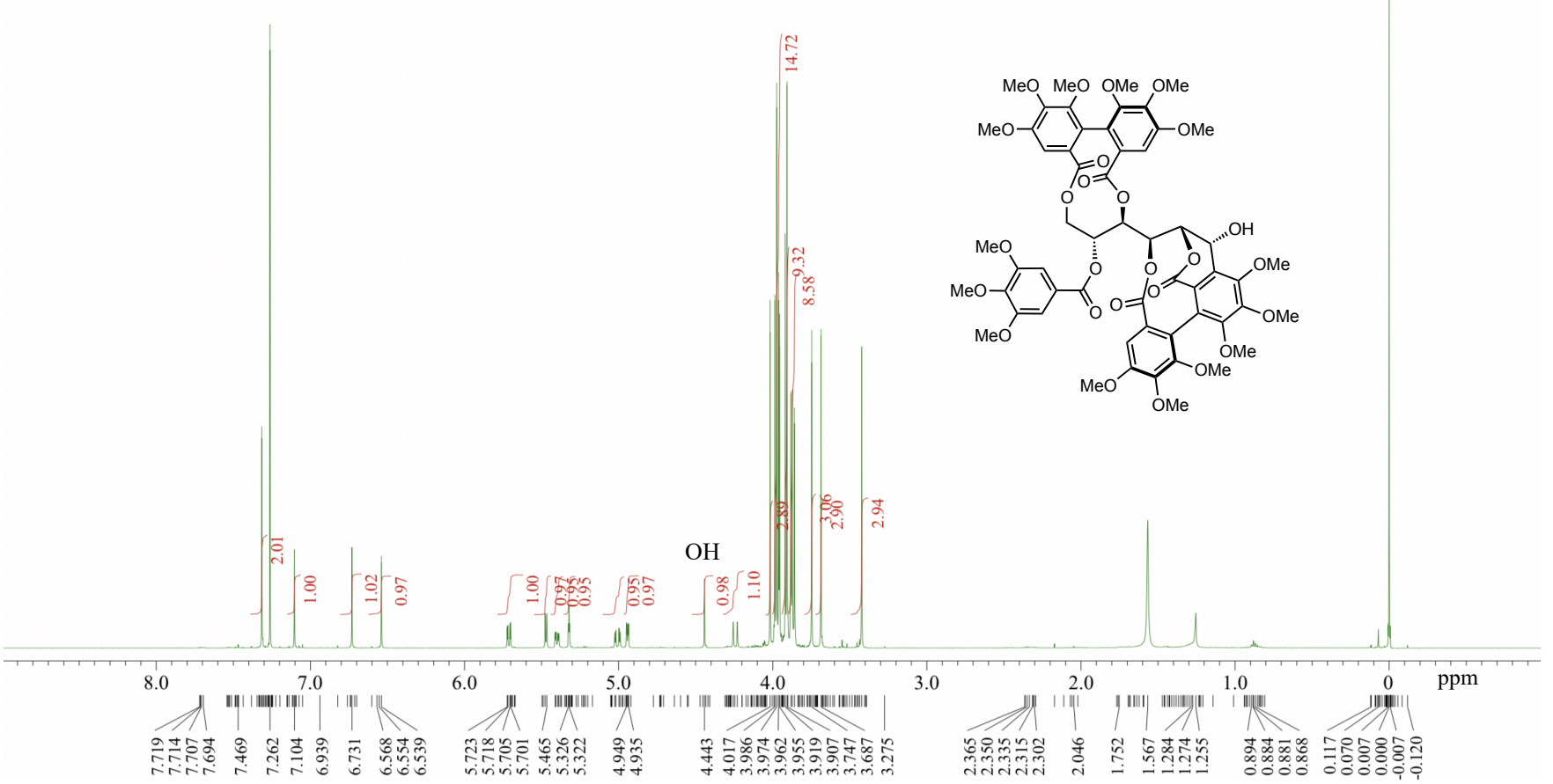


${ }^{1} \mathrm{H}$ NMR $\left(500 \mathrm{MHz}, \mathrm{CDCl}_{3}+\mathrm{D}_{2} \mathrm{O}\right)$

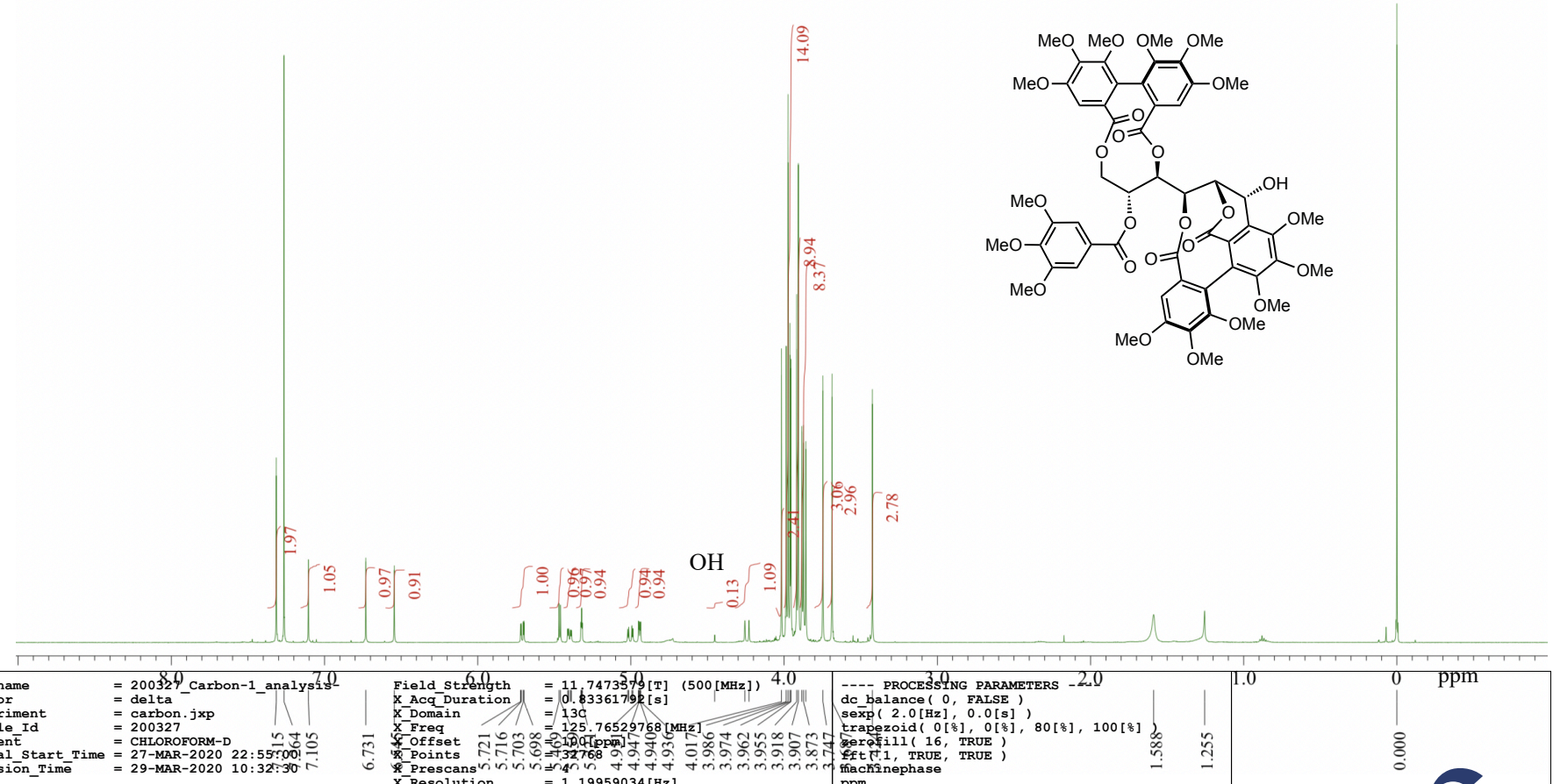

${ }^{13} \mathrm{C} \mathrm{NMR}\left(126 \mathrm{MHz}, \mathrm{CDCl}_{3}\right)$

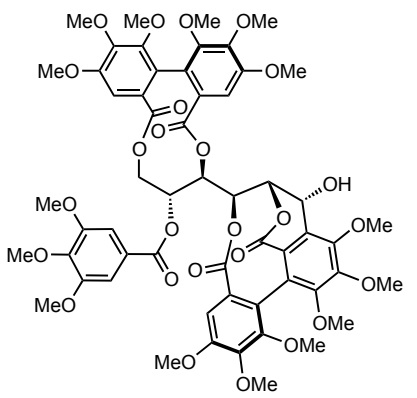

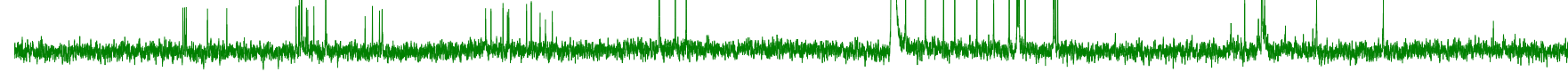

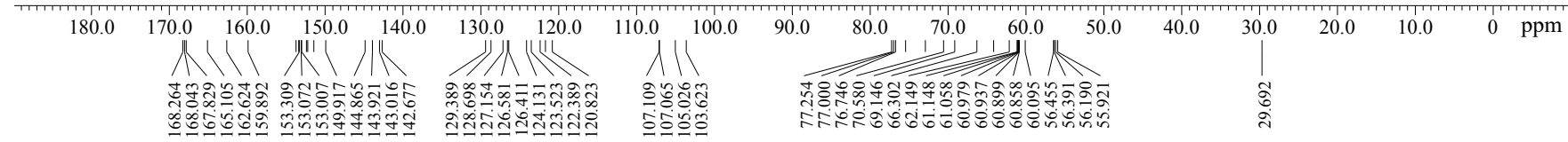


SI-33 Ratio in 23 and 24 in the incompletely separated fraction (see SI-09)

${ }^{1} \mathrm{H}$ NMR $\left(500 \mathrm{MHz}, \mathrm{CDCl}_{3}\right)$

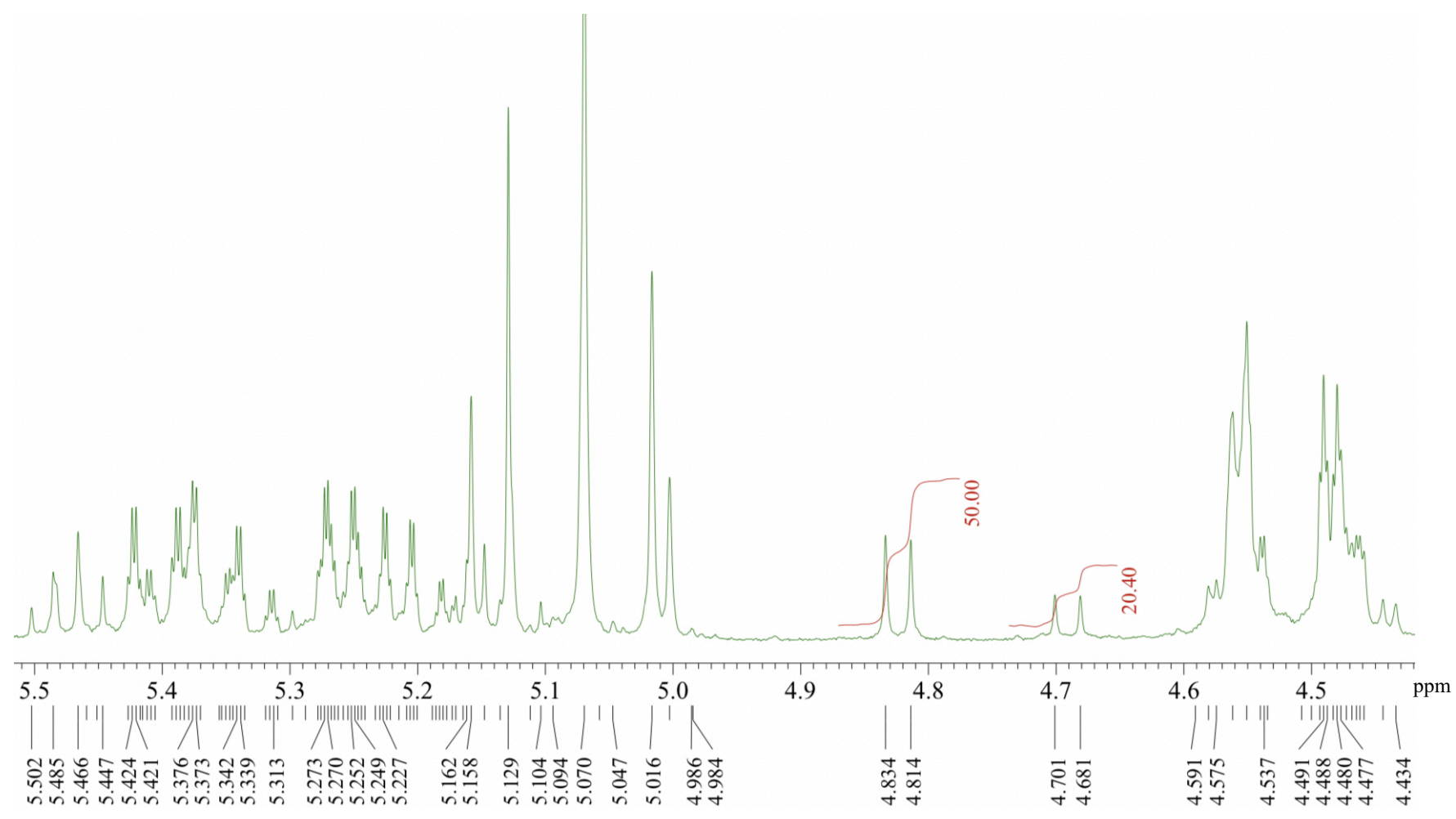




\section{SI-34 Comparison spectra of 23 (reactant) with that of 23 (recovered)}

${ }^{1} \mathrm{H}$ NMR (500 MHz, $\mathrm{CDCl}_{3}$ )

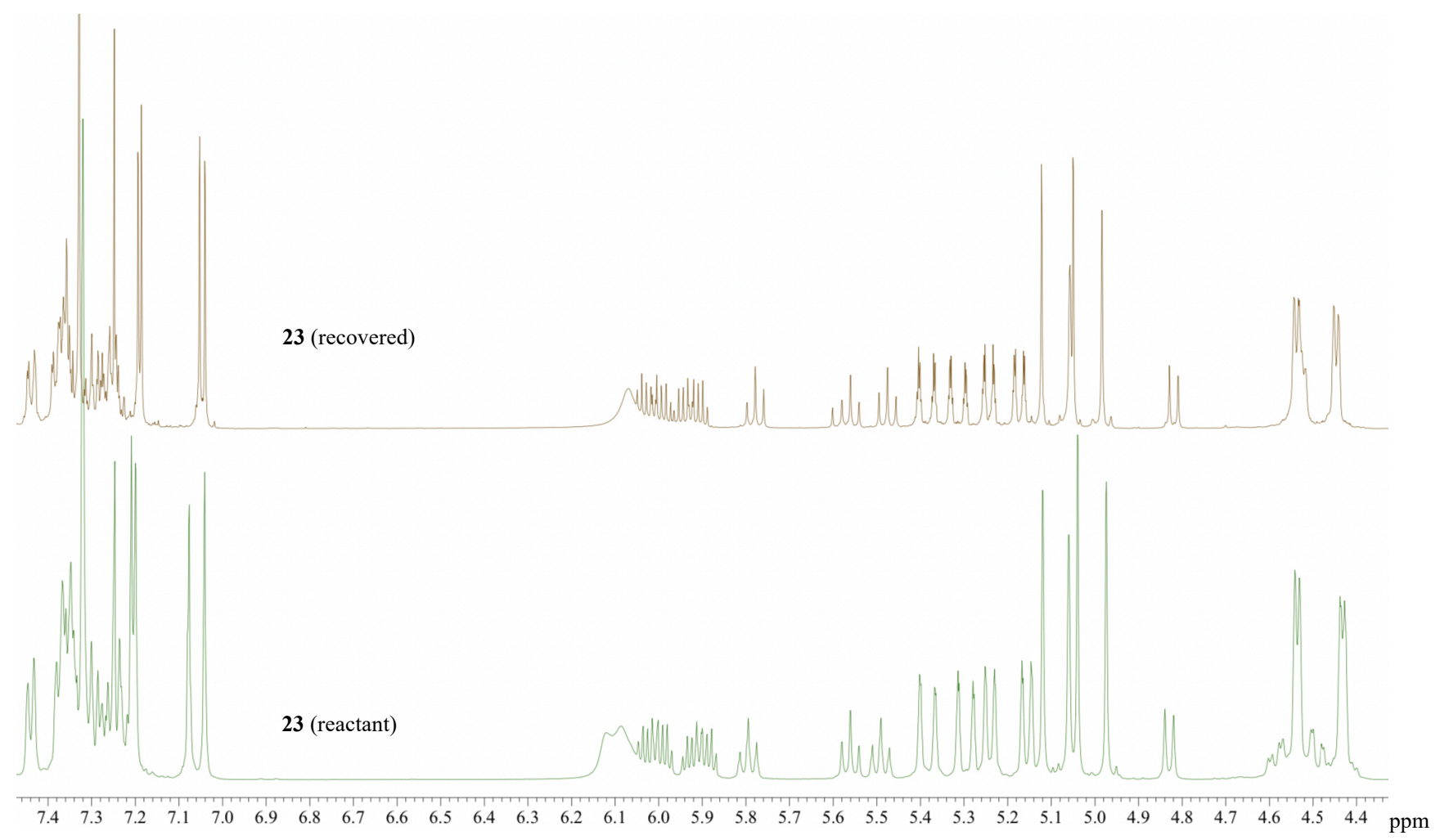

${ }^{13} \mathrm{C} \mathrm{NMR}\left(126 \mathrm{MHz}, \mathrm{CDCl}_{3}\right)$
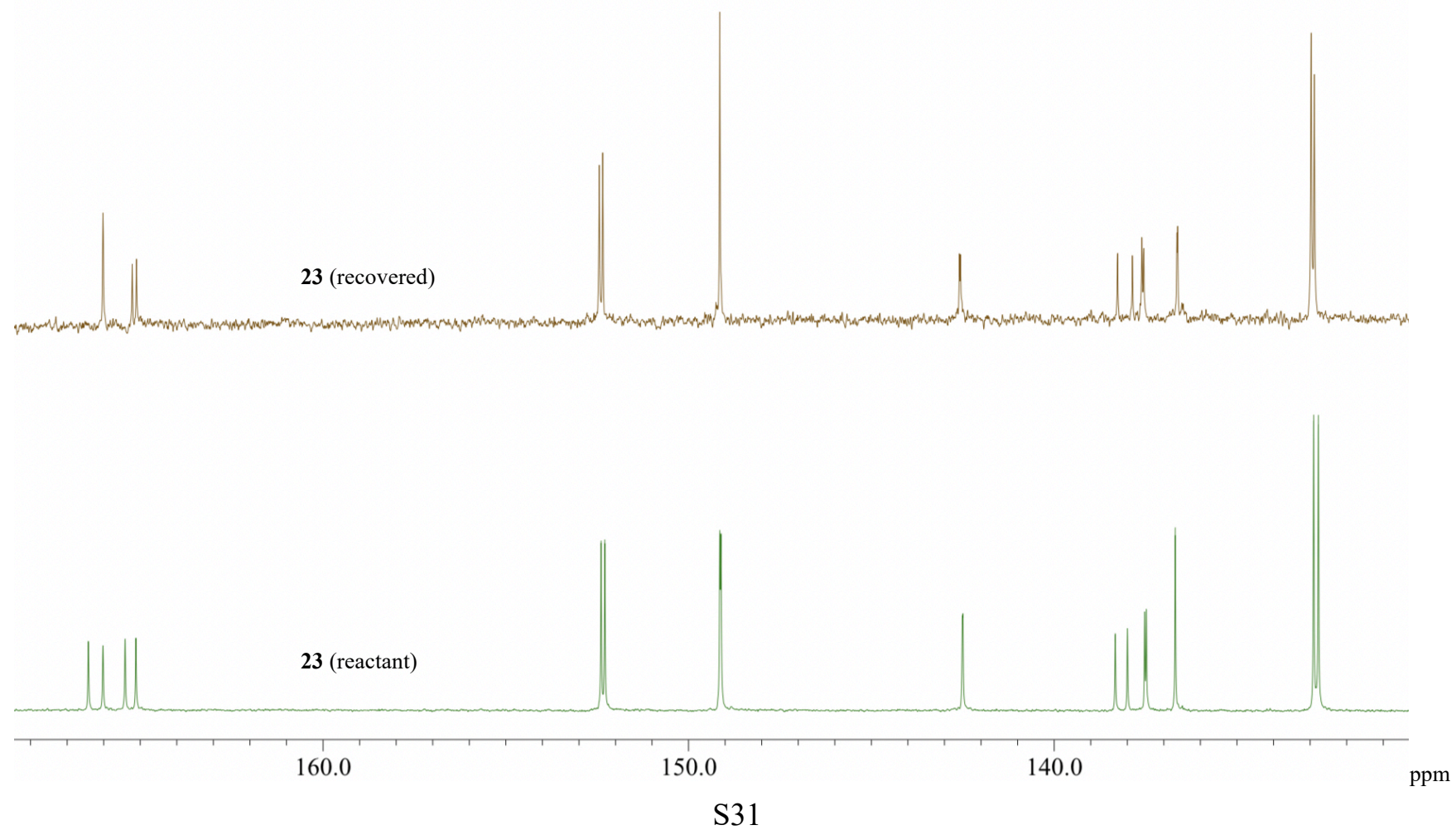
IR (ATR)
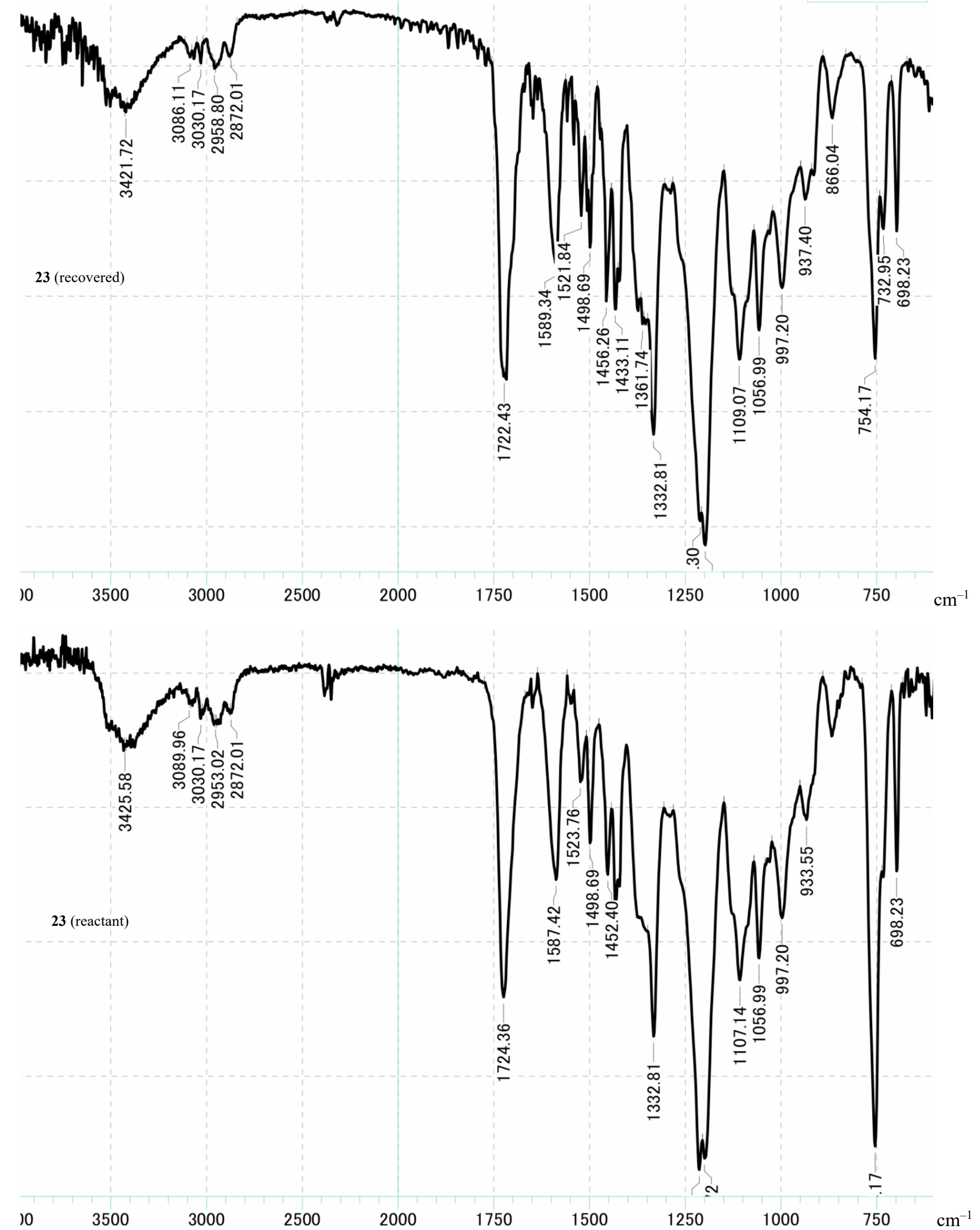

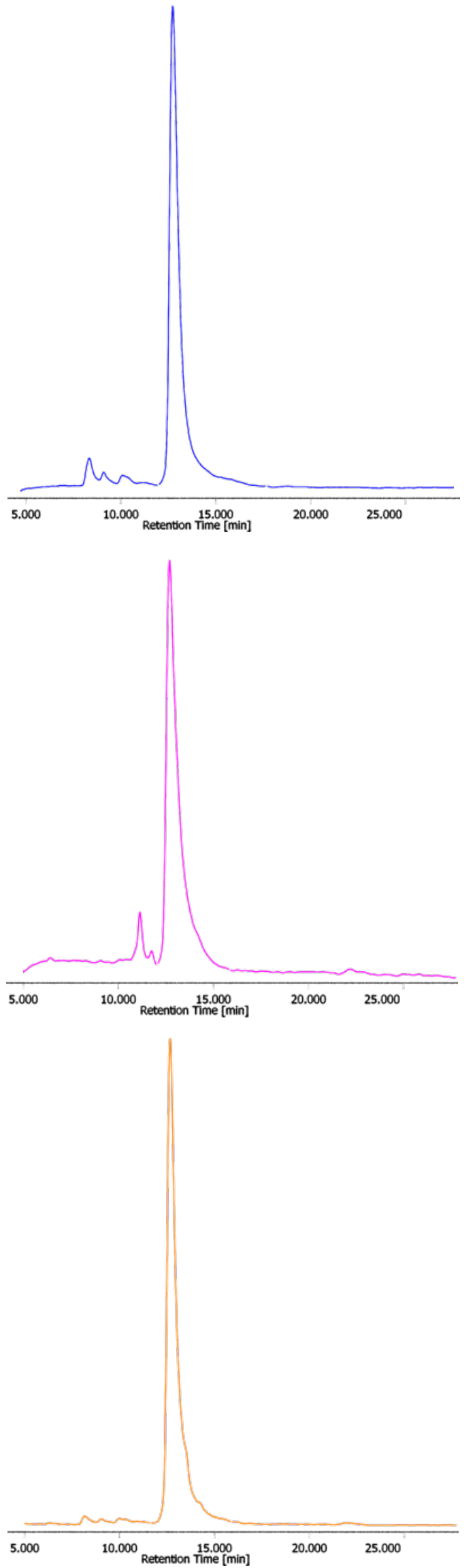

Sample

Retention time

Column

Solvent

Flow rate

Temperature

Detector

Sample

Retention time

Column

Solvent

Flow rate

Temperature

Detector

Sample

Retention time

Column

Solvent

Flow rate

Temperature

Detector
23 (recovered)

$14.8 \mathrm{~min}$

YMC-Pack SIL

$4.6 \mathrm{mmID} \times 250 \mathrm{mmL}, 5 \mu \mathrm{m}, 12 \mathrm{~nm}$ hexane $/$ EtOAc $=70 / 30$

$1 \mathrm{~mL} / \mathrm{min}$

$29^{\circ} \mathrm{C}$

UV $254 \mathrm{~nm}$

23 (reactant)

$14.8 \mathrm{~min}$

YMC-Pack SIL

$4.6 \mathrm{mmID} \times 250 \mathrm{mmL}, 5 \mu \mathrm{m}, 12 \mathrm{~nm}$ hexane $/ \mathrm{EtOAc}=70 / 30$

$1 \mathrm{~mL} / \mathrm{min}$

$29^{\circ} \mathrm{C}$

UV $254 \mathrm{~nm}$

$\mathbf{2 3}($ recovered $)+\mathbf{2 3}$ (reactant $)$

$14.8 \mathrm{~min}$

YMC-Pack SIL

$4.6 \mathrm{mmID} \times 250 \mathrm{mmL}, 5 \mu \mathrm{m}, 12 \mathrm{~nm}$ hexane $/ \mathrm{EtOAc}=70 / 30$

$1 \mathrm{~mL} / \mathrm{min}$

$29^{\circ} \mathrm{C}$

UV $254 \mathrm{~nm}$ 
SI-35 Ratio in the sulfoxides (see SI-13)

${ }^{1} \mathrm{H}$ NMR (500 MHz, $\mathrm{CDCl}_{3}$ )

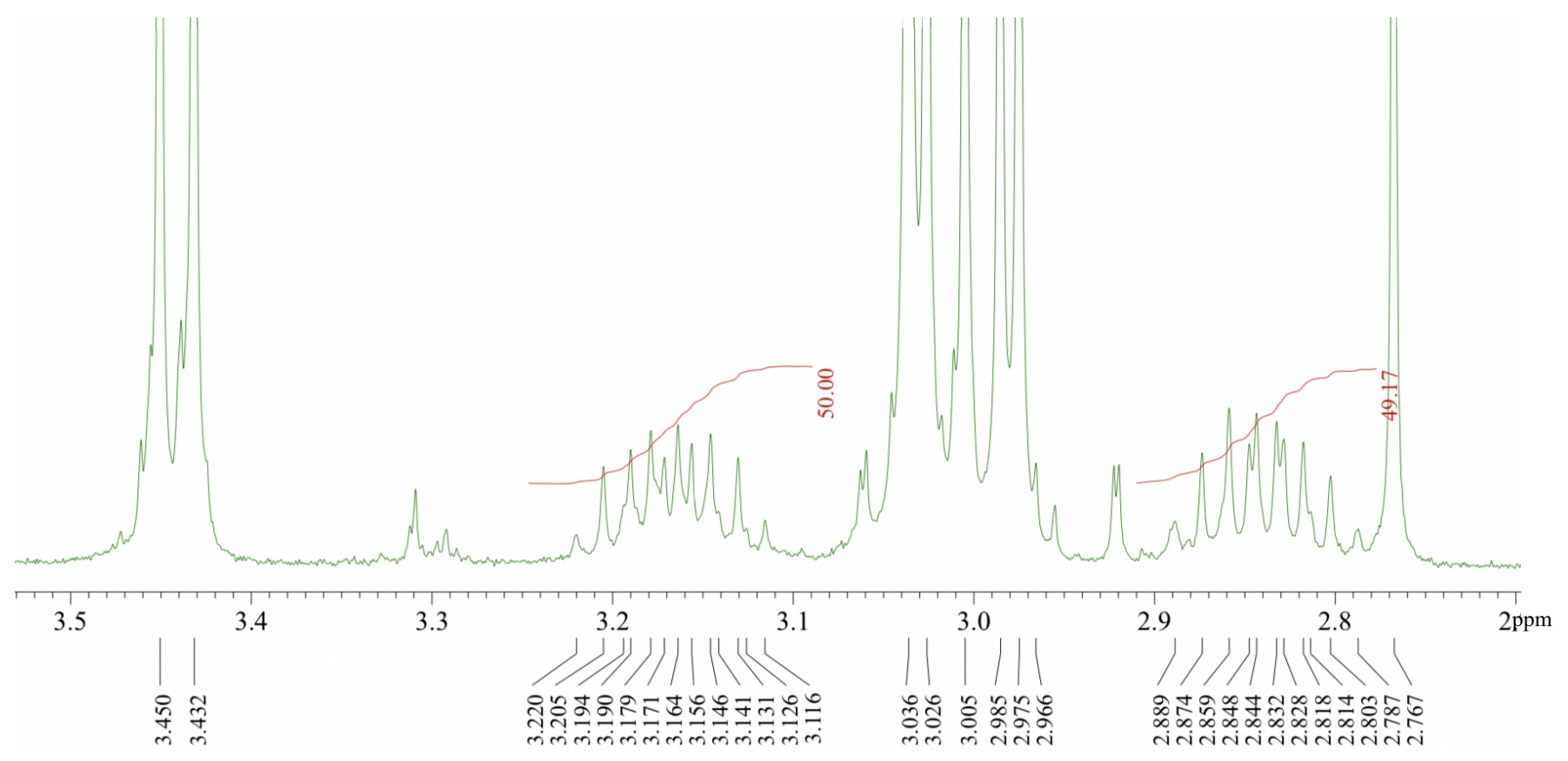

SI-36 Ratio in the oximes (see SI-15)

${ }^{1} \mathrm{H} \mathrm{NMR}\left(500 \mathrm{MHz}, \mathrm{CDCl}_{3}\right)$

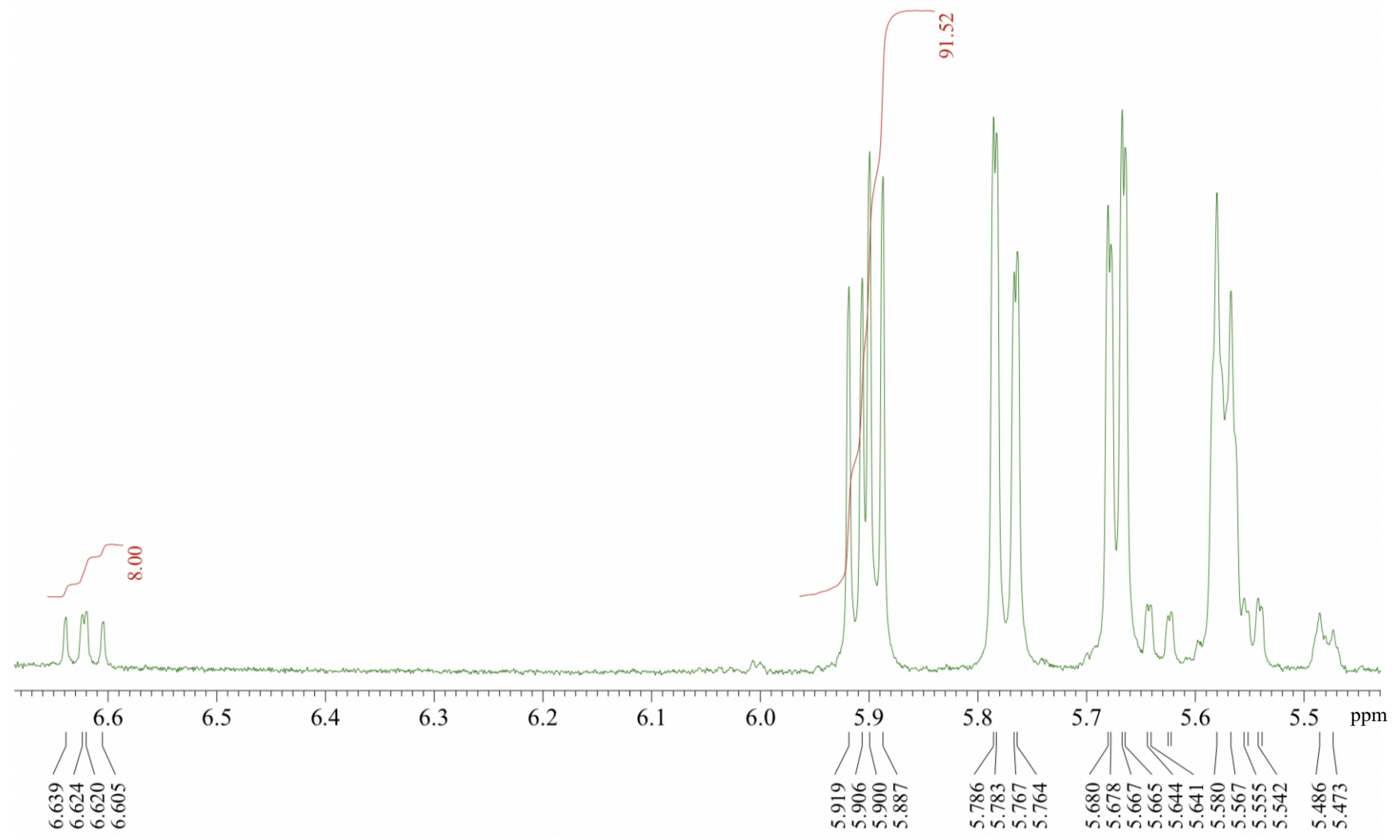




\section{SI-37 MS spectra of the hydrolysate of 29}

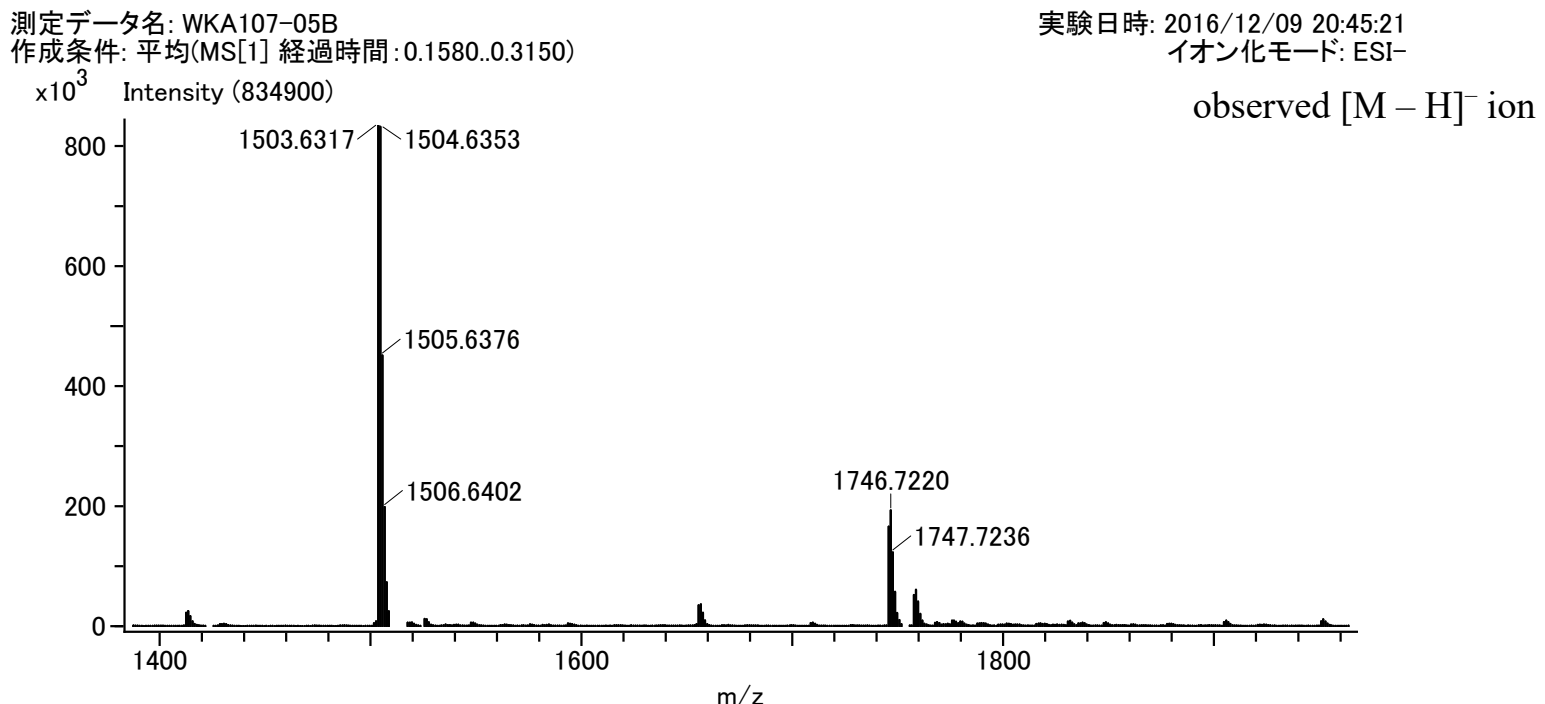

組成式: $\mathrm{C} 9 \mathrm{OH} 72 \mathrm{O} 22$

モノアイソトピック質量: 1503.4436962

付加/脱離イオン: - $-\mathrm{H}+$

Intensity (\%)
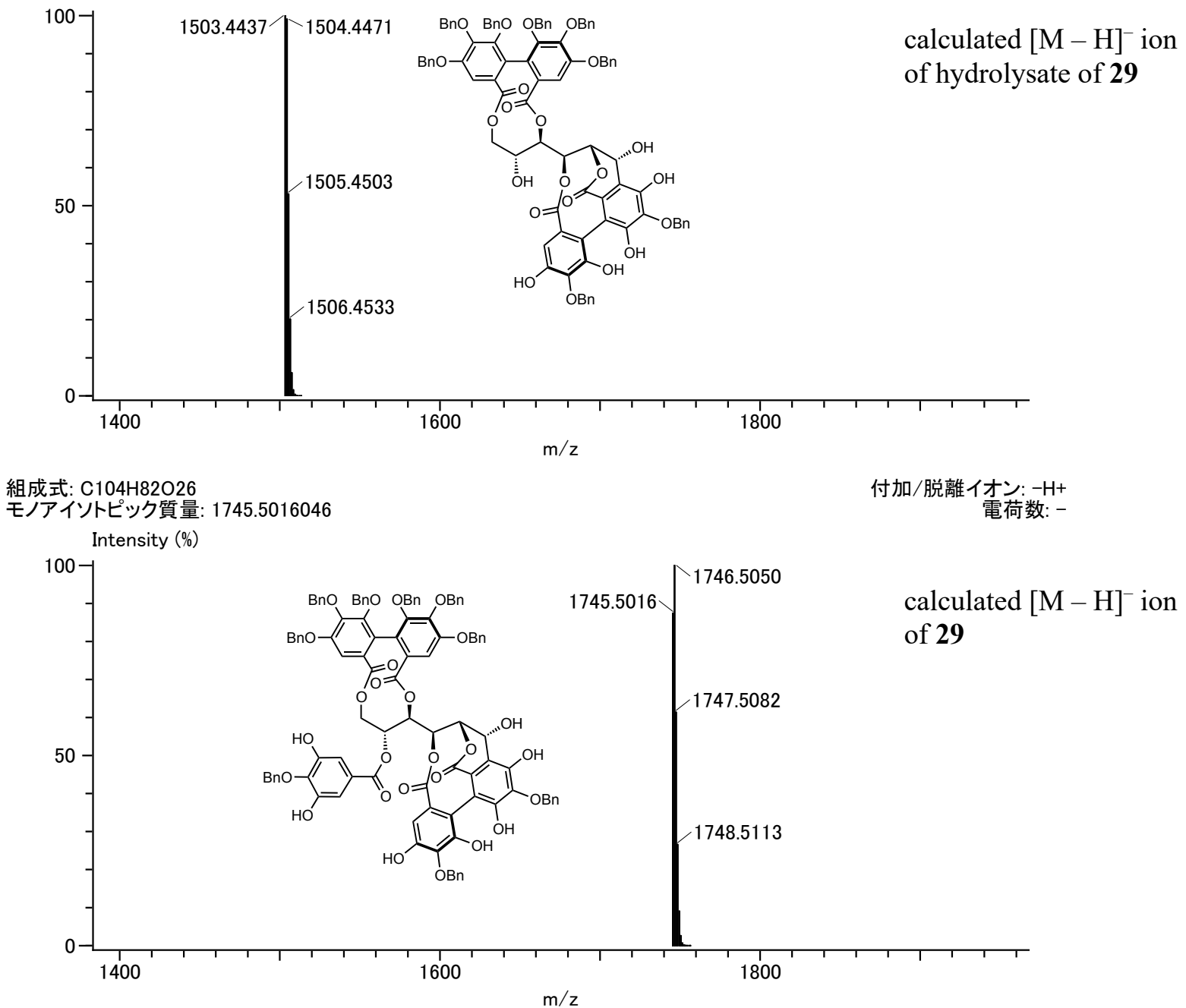


\section{Selected Reference}

(24) Bochkov, A. F.; Dzhein, A. Ch. Bull. Acad. Sci. USSR, Div. Chem. Sci. (Engl. Transl.) 1968, 168.

(25) Yamaguchi, S.; Ashikaga, Y.; Nishii, K.; Yamada, H. Org. Lett. 2012, 14, 5928.

(26) Yamada, H.; Nagao, K.; Dokei, K.; Kasai, Y.; Michihata, N. J. Am. Chem. Soc. 2008, 130, 7566.

(37) Okuda, T.; Yoshida, T.; Ashida, M.; Yazaki, K. J. Chem. Soc., Perkin Trans. 1 1983, 22, 1765. 